Dissertations. No. 1729

\title{
Security-Driven Design of Real-Time Embedded Systems
}

\author{
by
}

Ke Jiang

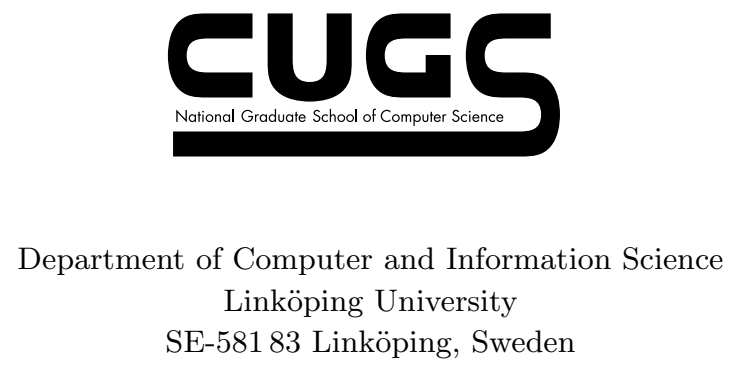

Linköping 2016 
Copyright (c) Ke Jiang 2016

ISBN 978-91-7685-884-4

ISSN 0345-7524

Printed by LiU Tryck 2015

URL: http://urn.kb.se/resolve?urn=urn:nbn:se:liu:diva-123016 
献给我的家人 



\section{Abstract}

$\mathrm{R}^{\text {EAL-TIME embedded systems (RTESs) have been widely used in }}$ $R$ modern society. And it is also very common to find them in safety and security critical applications, such as transportation and medical equipment. There are, usually, several constraints imposed on a RTES, for example, timing, resource, energy, and performance, which must be satisfied simultaneously. This makes the design of such systems a difficult problem.

More recently, the security of RTESs emerges as a major design concern, as more and more attacks have been reported. However, RTES security, as a parameter to be considered during the design process, has been overlooked in the past. This thesis approaches the design of secure RTESs focusing on aspects that are particularly important in the context of RTES, such as communication confidentiality and side-channel attack resistance.

Several techniques are presented in this thesis for designing secure RTESs, including hardware/software co-design techniques for communication confidentiality on distributed platforms, a global framework for secure multi-mode real-time systems, and a scheduling policy for thwarting differential power analysis attacks.

All the proposed solutions have been extensively evaluated in a large amount of experiments, including two real-life case studies, which demonstrate the efficiency of the presented techniques.

The research presented in this thesis has been funded by CUGS (the National Graduate School in Computer Science in Sweden) and VR (the Swedish Research Council). 



\section{Populärvetenskaplig Sammanfattning}

NBYGGDA realtidssystem används idag i stor utsträckning i vårt 1 moderna samhälle. Det är mycket vanligt att hitta dem i säkerhetskritiska tillämpningar såsom transport- och medicinsk utrustning. Vanligtvis har inbyggda realtidssystem flera designbegränsningar och krav gällande till exempel timing, resurser, energi och prestanda, som måste vara uppfyllda samtidigt. Detta gör utformningen av sådana system till ett mycket svårt problem.

Under senare tid har säkerheten i inbyggda realtidssystem blivit en viktig konstruktionsaspekt eftersom fler och fler attacker har rapporterats. Tidigare har säkerhetskraven i inbyggda realtidssystem inte betraktats som designparametrar under själva designprocessen. Denna avhandling fokuserar bland annat på utformningen av säkra inbyggda system, huvudsakligen på aspekter som är särskilt viktiga i samband med realtidssystem, till exempel kommunikationssekretess och motstånd mot sidokanalattacker.

I denna avhandling presenteras tre olika tekniker för att utforma säkra inbyggda realtidssystem. Först en teknik för hård- och mjukvaru co-design för kommunikationssekretess för distribuerade plattformar, sedan ett globalt ramverk för säkra multi-mode system och slutligen en schemaläggningspolicy för motstånd mot sidokanalattacker.

Samtliga de föreslagna lösningarna har utvärderats i stor omfattning med en stor mängd experiment, inklusive två fallstudier som visar effektiviteten hos de presenterade teknikerna. 



\section{Acknowledgements}

First and foremost, I must say that it is such a fortune to work with my supervisors, Prof. Zebo Peng and Prof. Petru Eles, who are the greatest advisers I could ever imagine and the most knowledgeable people I have ever met. When I got lost in the vast sea of research, it has always been your vision, faith, and patience that helped me through. Doing a $\mathrm{PhD}$ is the best decision I have ever made.

Thank you, Zebo, for taking me into the exciting world of research and giving me the most generous supports and guidance. When encountering problems, you are the first person I would turn to for help. I sincerely appreciate your advices both in research and private life.

Thank you, Petru, for "grilling" me (in absolutely the good sense) in our technical discussions, which is one of the main forces pushing the progress. You are not only a role model of mine but also a walkingencyclopedia reminding me how little I know about the world.

Thank you, Prof. Lejla Batina, for broadening my knowledge in security and inviting me to the extraordinary summer school. It has always been enjoyable discussing with you. Thank you, Prof. Wei Jiang, for sharing all research ideas, as well as being a good friend giving me advices in private life. Thank you, Prof. Jörg Keller, for inviting me to visit you and treating me with high hospitality.

I would like to thank the administrative staff at IDA, especially Eva Pelayo Danils, Åsa Kärrman, Inger Norén, Anne Moe, Marie Johansson, and Inger Emanuelsson for helping me with all kinds of issues. Life at IDA was much easier with your help.

The years in ESLAB is the most precious memory in my life. The group members are both colleagues and good friends to me. Thank 
you, Adi and Amir, for being the best office neighbors. You are always the reliable source when I want to talk about anything, either technical or personal. Thank you, Sudi, Unmesh, and Ahmed, for the discussions that inspired me in different projects. Your passions and dedications for research are something I sincerely admire.

Thank you, Dimitar and Breeta, for helping me adjust work/life balance. Thank you, Soheil and Urban, for advising me in job related issues. Thank you, Bogdan and Sergiu, for the cheering conversations and technical supports. Thank you, Arian, Nima, Ivan, Adrian, and Farrokh for all the interesting random topics we talked about over lunch as well as being responsive in all aspects.

I also want to show my appreciation to Erik, for proofreading my "populärvetenskaplig sammanfattning".

My life would not be exciting without my friends in private life. I want to express my deepest gratitude to all of you, especially the ones in Linköping, Norrköping, Stockholm, Västerås, and Oslo. Thank you so much for the joys and funs you shared with me. Our friendship is what I do and will always treasure.

Last but not least, I would like to thank my family. Thank you, my mother Jianhua and my father Aibao, for giving me infinite love and selfless supports through my entire life. You have sacrificed too much for letting me pursue my dreams. So I want to dedicate this thesis to you. Thank you, my little princess Miaohan, for being the most wonderful chapter in my life. Your smile is so beautiful and innocent, and is worth everything to see it. Finally, thank you very much, my beloved wife Li, for your unconditional love. You made me the luckiest man in the world. Thank you for taking care of everything when I was busy with work. Without your supports, I could not have come this far. Thank you, and I love you.

KE JIANG

Linköping

Dec. 2015 


\section{Contents}

1 Introduction 1

1.1 Motivation ................. 1

1.2 Summary of Contributions . . . . . . . . . . . . 3

1.3 List of Publications . . . . . . . . . . . . . . . 4

1.4 Thesis Organization $\ldots \ldots \ldots \ldots$

2 Background and Related Work 9

2.1 Embedded System Design . . . . . . . . . . . . . . . . 9

2.2 Design Requirements . . . . . . . . . . . . . . . . 12

2.2.1 Energy Efficiency . . . . . . . . . . . . . . 12

2.2 .2 Timeliness . . . . . . . . . . . . . . . . . . . 13

2.2.3 Multi-Mode Operation . . . . . . . . . . . . 13

2.3 Secure Embedded Systems Design _. . . . . . . . . 14

2.3.1 Cryptography and Other Security Services . . . 15

2.3.2 Side-Channel Attacks and Protections . . . . . 17

3 Preliminaries $\quad 19$

3.1 Hardware Architecture and Power Model . . . . . . . 19

3.1.1 Hardware Architecture . . . . . . . . . . . . . . 19

3.1 .2 Power Model . . . . . . . . . . . . . . . . . 21

3.2 Security in Embedded Systems . . . . . . . . . . 22

3.2 .1 Confidentiality . . . . . . . . . . . . . 22

3.2.1.1 Iterated Block Ciphers . . . . . . . . 24

3.2.1.2 Protection Strength of IBC . . . . . . 25

3.2.1.3 Side-Channel Attacks on IBC . . . . . 26

$3.2 .2 \quad$ Intrusion Detection . . . . . . . . . . . . . 30 
4 Design of Secure Distributed Embedded Systems 33

4.1 System Model . . . . . . . . . . . . . . . . . . 34

4.1.1 Architecture Model . . . . . . . . . . . . . . . . 34

4.1 .2 Application Model . . . . . . . . . . . . . . . 34

4.2 Confidentiality Optimization . . . . . . . . . 35

4.2.1 Motivational Example . . . . . . . . . . . 36

4.2 .2 Problem Formulation . . . . . . . . . . . 38

$4.2 .2 .1 \quad$ Step I . . . . . . . . . . . . . . . . . . 39

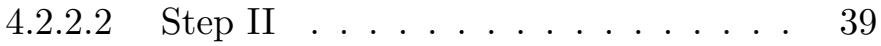

4.2 .3 Proposed Techniques . . . . . . . . . . . . . 40

4.2.3.1 CLP Formulation . . . . . . . . . . 41

4.2.3.2 Heuristic Approach . . . . . . . . . 43

4.2.4 Experimental Results . . . . . . . . . . . . 45

4.2.5 A Real-Life Case Study . . . . . . . . . . . . 48

4.3 Implementation Optimization . . . . . . . . . . . 50

4.3.1 Motivational Example . . . . . . . . . . . . 51

4.3.2 Problem Formulation _. . . . . . . . . . 55

4.3.3 Proposed Techniques . . . . . . . . . . . 56

4.3.3.1 FPGAs with Static Configuration . . 56

4.3.3.2 Partial Dynamic Reconfiguration . . . 62

4.3.4 Experimental Results . . . . . . . . . . . . 64

4.3.4.1 FPGA with Static Configuration . . . 64

4.3.4.2 Partial Dynamic Reconfiguration . . . 66

4.4 Summary . . . . . . . . . . . . . . . . 68

5 Design of Secure Multi-Mode Embedded Systems $\quad 69$

5.1 System Model . . . . . . . . . . . . . . . . 70

5.1 .1 Hardware Model . . . . . . . . . . . . . . 70

5.1 .2 Application Model . . . . . . . . . . . . . 71

5.1.2.1 Task Model . . . . . . . . . . . . 71

5.1.2.2 Execution Modes . . . . . . . . . . . . 72

5.1.3 Scheduling Model . . . . . . . . . . . . . . . 73

5.2 Design Objectives . . . . . . . . . . . . . . . . . 74

5.2 .1 Quality of Service . . . . . . . . . . 74

5.2 .2 Quality of Confidentiality . . . . . . . . 75

5.2 .3 Intrusion Detection Accuracy . . . . . . . . . 76 
5.2.4 Average Power Consumption _. . . . . . . 76

5.3 Motivational Example . . . . . . . . . . . . . . 78

5.4 Problem Formulation . . . . . . . . . . . . . 85

5.4.1 Design-Time Optimization . . . . . . . . . 85

5.4.1.1 Multi-Objective Optimization for A Given Mode . . . . . . . . . . . 86

5.4.1.2 Hasse Diagram Exploration . . . . . . 87

5.4.1.3 Selection of Candidate Modes . . . . 88

5.4 .2 Run-Time Optimization . . . . . . . . . . . 88

5.5 Proposed Techniques . . . . . . . . . . . . . . 89

5.5.1 Design-Time (Off-line Phase) . . . . . . . . . 91

5.5.1.1 Multi-Objective Optimization for A Given

Mode ... . . . . . . . . . . 91

5.5.1.2 Hasse Diagram Exploration . . . . . . 91

5.5.1.3 Selection of Candidate Modes . . . . 94

5.5.2 Run-Time (On-line Phase) . . . . . . . . . 95

5.5.2.1 $\quad M \in \mathcal{M}^{m e m} \ldots \ldots \ldots \ldots 95$

$5.5 .2 .2 \quad M \notin \mathcal{M}^{m e m} \ldots \ldots \ldots . \ldots 96$

5.6 Experimental Results . . . . . . . . . . . . . . . . . . 98

5.6 .1 Design-Time . . . . . . . . . . . . . . . . . . . 98

5.6 .2 Run-Time . . . . . . . . . . . . . . . . . . . 101

5.7 A Real-Life Case Study . . . . . . . . . . . . . . . 104

5.8 Summary . . . . . . . . . . . . . . . . . . . 108

6 Design of A Secure Scheduler Against Differential Power Analysis Attacks 111

6.1 System Model . . . . . . . . . . . . . . . . . . . . 112

6.1 .1 Hardware Model . . . . . . . . . . . . . . . . 112

6.1.2 Application Model . . . . . . . . . . . . . . . . 113

6.2 Time Dimension Shuffling Based Countermeasures . . 113

6.3 Motivational Example . . . . . . . . . . . . . . 115

6.4 Proof of Concept on Existing Schedulers . . . . . . . 117

6.4.1 Evaluation under Different Processor Utilizations118

6.4.2 Evaluation on Different Problem Sizes . . . . . 119

6.4.3 Evaluation on the Same Problem Size . . . . . 120

6.5 A New Scheduling Policy for Thwarting DPA Attacks 120 
6.5.1 Proposed Scheduler: SPARTA _. . . . . . . 121

6.5.2 Properties of SPARTA . . . . . . . . . . . . . 124

6.5.2.1 Schedulability Guarantee . . . . . . . 124

6.5.2.2 Upper-bound of Context Switches . . 126

6.5.2.3 Complexity of SPARTA . . . . . . . 130

6.6 Experimental Evaluation . . . . . . . . . . . . . 131

6.6.1 Different Processor Utilizations . . . . . . . . . 131

6.6.2 Different Problem Sizes . . . . . . . . . . . . 132

6.7 Summary . . . . . . . . . . . . . . . . . . . . 133

7 Conclusions and Future Work 135

7.1 Conclusions . . . . . . . . . . . . . . . 135

7.1.1 Confidentiality-Aware Design Techniques . . . 136

7.1.2 Secure Multi-Mode RTES Design Framework . 136

7.1.3 Scheduling Against Side-Channel Attacks . . . 137

7.2 Future Work . . . . . . . . . . . . . . . . . 137 


\section{List of Figures}

2.1 System design flow . . . . . . . . . . . . . . 10

3.1 An overall hardware architecture . . . . . . . . 20

3.2 Calculate correlations between power measurements and

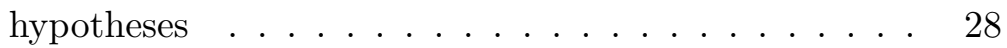

4.1 A simple application with 5 tasks . . . . . . . 35

4.2 A processor-based platform . . . . . . . . . 36

4.3 Schedule without cryptographic protections . . . . . 37

4.4 Schedule with RC6-20 on all messages . . . . . . . 37

4.5 Schedule after balanced maximization on all messages 37

4.6 Schedule after distribution of the slacks from Figure 4.5 37

4.7 Reconstructed task graph of Figure 4.1 . . . . . . . 40

4.8 New task mapping from Figure $4.2 \ldots \ldots$. . . . . . . 41

4.9 Average execution time of finished CLP experiments and heuristic approach . . . . . . . . . . 47

4.10 Result comparison of step I . . . . . . . . . . . . 47

4.11 Result comparison of step II . . . . . . . . . . . . 47

4.12 An adaptive cruise controller . . . . . . . . . . . . . 49

4.13 Hardware architecture of ACC . . . . . . . . . . . 49

4.14 Another illustrative application . . . . . . . . . 52

4.15 An FPGA-accelerated architecture . . . . . . . . . 52

4.16 Schedule without cryptographic protections . . . . . 54

4.17 Schedule of software only solution . . . . . . . . . 54

4.18 Schedule of assigning FPGA to all E/Ds . . . . . . . 54

4.19 Schedule of the optimal solution for static FPGA . . . 54 
4.20 Schedule of the optimal solution for PDR-enable FPGA 54

4.21 Reconstructed task graph of Figure $4.14 \ldots \ldots$. . . . 57

4.22 An example with 3 consecutive messages . . . . . . . . 60

4.23 Optimization time with statically configured FPGA . 65

4.24 Results with statically configured FPGA (1) . . . . . 65

4.25 Results with statically configured FPGA (2) . . . . . 65

4.26 Optimization time with PDR enabled FPGA . . . . 67

4.27 Results with PDR enabled FPGA (1) . . . . . . . 67

4.28 Results with PDR enabled FPGA $(2) \ldots \ldots 7$

5.1 An illustrative multi-mode system . . . . . . . . 70

5.2 The Hasse diagram of modes for Figure $5.1 \ldots 72$

5.3 Pareto space of mode $M^{134} \ldots \ldots \ldots$. . . . . . 81

5.4 Derived solution space for mode $M^{13}$ from $M^{123}$. . 82

5.5 Derived solution space for mode $M^{13}$ from $M^{134}$. . . 82

5.6 Derived solution space for mode $M^{13}$ from $M^{1234}$. . . 82

5.7 Overall flow diagram of our proposed design framework 90

5.8 Performance improvement of off-line phase . . . . . . . 100

5.9 Optimization time of off-line phase . . . . . . . . . 100

5.10 Average time of solving ILP . . . . . . . . . . . . 100

5.11 Average distance (\%) from solutions on Pareto spaces 102

5.12 Average performance improvement over greedy method 102

5.13 Average time of on-line operation point adaptation . . 102

5.14 The off-line and on-line optimization overheads . . . . 108

6.1 An illustrative system . . . . . . . . . . . . . . . 112

6.2 Power traces of AES on two messages with the same key114

6.3 (a) The system schedule under EDF; (b) The aligned samples of $(\mathrm{a}) \ldots \ldots \ldots \ldots \ldots$

6.4 (a) Another system schedule; (b) The aligned samples of $(\mathrm{a}) \ldots \ldots \ldots \ldots \ldots \ldots \ldots$

6.5 (a) Random schedule of $\mathcal{H P}$; (b) Random schedule for $\mathcal{H P}^{\prime}$; (c) The aligned samples of (a) and (b) . . . . . 116

6.6 Results of 5 tasks under different processor utilizations 119

6.7 Results of different problem sizes . . . . . . . . . . 119 
6.8 Robustness $R\left(\operatorname{Key}\left(\tau_{41}^{A E S}\right)\right)$ of 30 experiments under EDF and RMS . . . . . . . . . . . 120

6.9 An example schedule of $C S_{s}^{i=k}=k \ldots \ldots \ldots 127$

6.10 An example schedule of $C S_{s}^{i=k}=k+1 \ldots \ldots$. . . 127

6.11 An example schedule of $C S_{s}^{i=k}=k+2 \ldots \ldots . . .27$

6.12 Results of SPARTA on different processor utilizations 132

6.13 Results of SPARTA on different problem sizes . . . . . 133 


\section{List of Tables}

3.1 Protection strength and encryption time of different RC6 variants . . . . . . . . . . . . . 26

4.1 Results of different solutions in Figure 4.3-4.6 . . . . . 38

4.2 Result comparison of the ACC application . . . . . . 50

4.3 Results of different solutions in Figure 4.16-4.20 . . . 55

5.1 Task attributes for Figure $5.1 \ldots \ldots \ldots$

5.2 Task coexistence relations . . . . . . . . . . . 105

5.3 Task parameters of the smartphone benchmark . . . . 106

5.4 Experimental results of the smartphone benchmark . . 106

6.1 Task parameters for Figure $6.1 \ldots \ldots$. . . . . . 115 


\section{List of Abbreviations}

$\begin{array}{ll}\text { AAHE } & \text { Average Additional Hardware Expenditure } \\ \text { ACC } & \text { Adaptive Cruise Controller } \\ \text { AES } & \text { Advanced Encryption Standard } \\ \text { APC } & \text { Average Power Consumption } \\ \text { ASIC } & \text { Application-Specific Integrated Circuit } \\ \text { CAN } & \text { Controller Area Network } \\ \text { CLP } & \text { Constraint Logic Programming } \\ \text { CPS } & \text { Cyber-Physical Systems } \\ \text { CPU } & \text { Central Processing Unit } \\ \text { DPA } & \text { Differential Power Analysis } \\ \text { DVFS } & \text { Dynamic Voltage and Frequency Scaling } \\ \text { E/D } & \text { Encryption/Decryption } \\ \text { EDF } & \text { Earliest Deadline First } \\ \text { ES } & \text { Embedded System } \\ \text { ET } & \text { Execution Time } \\ \text { FPGA } & \text { Field Programmable Gate Array } \\ \text { GPU } & \text { Graphics Processing Unit } \\ \text { IBC } & \text { Iterated Block Cipher } \\ \text { ID } & \text { Intrusion Detection } \\ \text { IDA } & \text { Intrusion Detection Accuracy } \\ \text { ILP } & \text { Integer Linear Programming } \\ \text { LS } & \text { List Scheduling } \\ \text { PCP } & \text { Partial Critical Path } \\ \text { PDR } & \text { Partial Dynamic Reconfiguration } \\ \text { PI } & \text { Performance Improvement } \\ \text { QoC } & \text { Quality of Confidentiality } \\ & \end{array}$




$\begin{array}{ll}\text { QoS } & \text { Quality of Service } \\ \text { RDI } & \text { Random Delay Insertion } \\ \text { RMS } & \text { Rate-Monotonic Scheduling } \\ \text { RTES } & \text { Real-Time Embedded System } \\ \text { SA } & \text { Simulated Annealing } \\ \text { SCA } & \text { Side-Channel Attack } \\ \text { SCADA } & \text { Supervisory Control And Data Acquisition } \\ \text { SNR } & \text { Signal-to-Noise Ratio } \\ \text { SPARTA } & \text { Scheduling Policy for Thwarting Differential Power } \\ & \text { Analysis Attacks } \\ \text { TT } & \text { Transmission Time }\end{array}$




\section{List of Symbols}

\section{Tasks}

$\tau_{i}$

$c_{i}$

$\mathcal{P}_{i}$

$\mathcal{D}_{i}$

$\mathcal{E}_{i}^{m}$

$\mathcal{E}_{i}^{o}$

$c_{i}^{o}$

$\mathcal{Q}_{i}^{m}$

$\mathcal{F}_{i}$

$\mathcal{L}_{i}$

$m_{i j}$

$l_{i j}$

$w_{i j}$

$\mathcal{T}$

$\mathcal{H P}(\mathcal{T})$

$i^{\text {th }}$ task

Execution time of $\tau_{i}$

Release period of $\tau_{i}$

Relative deadline of $\tau_{i}$

Constant execution time of mandatory part of $\tau_{i}$

Maximal execution time of optional part of $\tau_{i}$

Actual execution time of optional part of $\tau_{i}$

Constant QoS reward from the mandatory part of $\tau_{i}$

QoS reward function from the optional part of $\tau_{i}$

Set of messages associated with $\tau_{i}$

$j^{\text {th }}$ message of $\tau_{i}$

Length (in number of blocks) of $m_{i j}$

Relative importance of $m_{i j}$

A set of tasks

Hyperperiod of all tasks in $\mathcal{T}$

\section{Dynamic Voltage and Frequency Scaling}

Pow

$C_{\text {eff }}$

$f_{i}$

$f^{\max }$

$V_{d d_{i}}$

$V_{d d}^{\max }$

$\left.\mathcal{W}_{i}\left(f_{i}\right)\right)$
Power consumption

Effective switching capacitance of $\tau_{i}$

Applied frequency on $\tau_{i}$

Maximal frequency of the processor

Supply voltage on $\tau_{i}$

Maximal supply voltage of the processor

Execution time of $\tau_{i}$ under frequency $f_{i}$ 


\section{Security Protections}

$\begin{array}{ll}k_{i} & \text { An AES subkey } \\ Q o C_{i j}^{\text {min }} & \text { Minimal required QoC on } m_{i j} \\ C_{i j} & \text { Chosen cipher (or variant) for protecting } m_{i j} \\ x_{i j} & \text { Number of IBC rounds of } C_{i j} \\ c e_{i} & \text { An encryption task } \\ c d_{i} & \text { A decryption task } \\ L P_{i} & \text { A leakage point } \\ \mathcal{R} & \text { Robostness of a secret key } \\ \hat{p} & \text { Probability of leakage occurrences } \\ \mathcal{E}_{I D} & \text { Execution time of the ID task } \\ \mathcal{P}_{I D}^{\min } & \text { Minimal required release period of the ID task } \\ \mathcal{P}_{I D} & \text { Actual release period of the ID task }\end{array}$

\section{Multi-Mode Systems}

$M^{i j \ldots k}$

$\mathcal{M}^{\text {func }}$

$\mathcal{M}_{\uparrow}^{\text {func }}$

$\overline{\mathcal{M}}(M)$

$\underline{\mathcal{M}}(M)$

$\mathcal{M}^{\text {impl }}$

$\mathcal{M}^{\text {mem }}$

$S_{M}$

$S_{M}^{M^{\prime}}$

$\mathcal{H}\left(S_{M}\right)$

$\mathcal{H}_{M}\left(\mathcal{M}^{\text {mem }}\right)$

$H$

Applications

G

E

$e_{i j}$

$p_{i}$
A mode in which tasks $\tau_{i}, \tau_{j}, \ldots$, and $\tau_{k}$ are active

Set of all functional modes

Set of top functional modes

Supermodes of $M$

Submodes of $M$

Candidates of Pareto spaces to be saved in memory

Set of Pareto spaces saved in memory

Pareto space for mode $M$

Derived solution space for mode $M$ from $M^{\prime}$

Hypervolume of Pareto space $S_{M}$

Hypervolume of mode $M$ with given pre-stored Pareto spaces $\mathcal{M}^{\text {mem }}$

Total hypervolume of all functional modes
Acyclic task graph

Edges of a task graph

The edge indicating dependency of $\tau_{j}$ on $\tau_{i}$

A computational unit 


\section{Chapter 1}

\section{Introduction}

$T^{\mathrm{HE} \text { focus of this thesis is on the design and scheduling of secure }}$ real-time embedded systems (RTESs). RTESs play a vital role in modern society and, due to their importance, guaranteeing the security of such systems emerges as a critical issue. The main contribution of this thesis is the development of several design techniques for achieving RTES security in the context of performance, energy, and resource constraints typical to such systems. In this chapter, we shall first introduce the fundamental motivations. Then we shall summarize the contributions and present the organization of this thesis.

\subsection{Motivation}

Real-time embedded systems have been applied in all aspects of our daily lives, from large scale control plants, e.g., the supervisory control and data acquisition (SCADA) systems, to consumer electronics. They usually consist of various processing units and peripherals, e.g., sensors and actuators. There are several design constraints imposed on a RTES, for example, timing, resource, energy, and performance constraints, which must be satisfied simultaneously. These constraints make the design of RTESs a difficult problem, and must be carefully considered together, since treating them separately cannot lead to a globally good solution.

Nowadays, it is common to find RTESs in safety and security 
critical areas such as transportation and health-care. The security of such systems is of critical importance, which, however, has been seriously overlooked in the past. This urges the need for providing RTES security, and is the most fundamental motivation for this thesis. For example, in automotive electronics systems [LH02], a typical distributed RTESs, the internal communication and message exchanges are done without any protections [WWP06], and are extremely easy to be eavesdropped and forged. Thus, it is indispensable to revisit the traditional RTES design approaches with security requirements considered as a critical factor. Because of the limited amount of available resources and yet stringent timing constraint, protecting RTES is a difficult problem, and straight-forward techniques cannot achieve satisfactory results. Thus, efficient mechanisms in terms of both solution quality and convergence time are expected. Chapter 4 presents our contributions to the design of secure distributed RTESs.

Many of the proposed techniques in the context of embedded systems security aim at finding lightweight cryptography or efficient implementations of cryptographic algorithms $\left[\mathrm{BMS}^{+} 06, \mathrm{BKL}^{+} 07, \mathrm{HS} 13\right]$. However, such techniques may not be able to cope with the timing and performance constraints and the increasing complexity of current RTESs. For example, RTESs are not anymore designed for a single dedicated usage but to operate under different modes. Furthermore, there could be multiple critical applications running simultaneously in the system, within each mode. Therefore, how to design such systems to be secure is not anymore a single-objective optimization problem. On the contrary, a design method taking all potential modes and design requirements into consideration is needed in order to achieve the best solution. Chapter 5 presents our contributions to the design of secure multi-mode RTESs.

Even a well designed RTES with sound cryptographic protections, i.e., by applying Advanced Encryption Standard (AES) on all the incoming and outgoing messages, may not be $100 \%$ secure. This is because the underlying implementations of the cipher algorithms can disclose sensitive information, e.g., power consumption and electromagnetic radiation, while operating. Such information can leak details about the actual implementation of the chosen cipher, such as the 
secret key, and may be exploited by the attacker to break the system (known as side-channel attacks). Such information leakage, rooted from the fundamental physical properties of the hardware platform, must be avoided or hidden in order to deliver the best protections. Chapter 6 introduces a scheduling-based technique to overcome the potential side-channel attacks.

\subsection{Summary of Contributions}

This thesis presents results towards several design optimization techniques for RTESs in which security is important. The major contributions of this thesis approach three representative design problems of secure RTESs, elaborated in Chapter 4, 5, and 6, respectively.

As revealed by literature [WWP06] the internal communication in many distributed RTESs containing sensitive information lacks even the basic security protection. In Chapter 4, we approach the problem of achieving secure communication within distributed RTESs. We first studied the problem of delivering the best confidentiality protection for the internal communication under the limited amount of available resources. Due to the complexity of this optimization problem, we present a heuristic approach to solve it. Then we look at a configuration in which the computational nodes consist not only of embedded processors but also of reconfigurable hardware, i.e., field-programmable gate arrays (FPGAs). We present two efficient heuristic-based techniques for finding the minimal hardware cost needed to implement the designated cryptographic algorithms for two FPGA technologies. The goal is to reduce the encryption and decryption overhead while satisfy timing constraints. The main challenge is to optimally utilize the available FPGA area.

In Chapter 5, we shall look at multi-mode RTESs. The main difficulty arises from the fact that, during run-time, the system can potentially function in a very large number of modes. Nevertheless, it is impossible to run, at run-time, complex optimizations in order to find an optimal setting from the point of view of timing, energy consumption, and security. We formulate an overall design problem for secure multi-mode RTESs as a two-stage optimization. A set of 
solutions are prepared in the off-line stage and are later used in the on-line stage to determine the best option to configure the system for the actual mode. In the off-line phase, the designer can trade-off solution quality with available design time and memory space. We evaluated the techniques in extensive experiments as well as a real-life case study. In addition, the presented techniques are general enough to be applied for different design demands besides the dimensions presented in the chapter.

The contributions mentioned above have focused on efficiently conducting the required computations including the security protection mechanisms, e.g., message encryption and decryption. However, the actual implementation of the chosen cipher algorithm may become the target of attacks. The well-known side-channel attacks (SCAs) aim to retrieve secret information of the underlying cryptographic implementations, for example, by observing the power consumption of the microprocessors. We found that several real-time scheduling policies are able to reinforce the security of cipher implementations, since they generate a certain amount of randomness in the power profile. Then, the question is how well a scheduler could act as a countermeasure against SCAs. In Chapter 6, we first present a metric for measuring the influence of a real-time scheduler on the robustness of AES secret keys under, arguably, the most popular and efficient type of SCAs, the differential power analysis attacks (DPAs). Then, we show that different scheduling policies have different impacts on the robustness of AES secret keys by evaluating two representative scheduling policies, i.e., earliest deadline first (EDF) and rate-monotonic scheduling (RMS). After that, we present a scheduling policy, i.e., SPARTA, for thwarting DPA attacks that shares the same guarantee of schedulability as EDF and, at the same time, counteracts against DPAs. SPARTA is the first real-time scheduler in literature specifically designed as a countermeasure against SCAs.

\subsection{List of Publications}

Parts of this thesis are presented in the following publications: 
- Ke Jiang, Petru Eles, and Zebo Peng. Optimization of Message Encryption for Distributed Embedded Systems with Real-Time Constraints. International Symposium on Design and Diagnostics of Electronic Circuits and Systems (DDECS), Cottbus, Germany, April 2011 ([JEP11])

- Ke Jiang, Petru Eles, and Zebo Peng. Co-Design Techniques for Distributed Real-Time Embedded Systems with Communication Security Constraints. Design, Automation Test in Europe (DATE), Dresden, Germany, March 2012 ([JEP12])

- Ke Jiang, Petru Eles, and Zebo Peng. Optimization of Secure Embedded Systems with Dynamic Task Sets. Design, Automation Test in Europe (DATE), Grenoble, France, March 2013 ([JEP13])

- Ke Jiang, Lejla Batina, Petru Eles, Zebo Peng. Robustness Analysis of Real-Time Scheduling Against Differential Power Analysis Attacks. IEEE Computer Society Annual Symposium on VLSI (ISVLSI), Tampa, FL, USA, July 2014 ([JBEP14])

- Ke Jiang, Petru Eles, Zebo Peng. Power-Aware Design Techniques of Secure Multi-Mode Embedded Systems. ACM Transactions on Embedded Computing Systems (TECS), 2015 ([JEP15])

- Ke Jiang, Petru Eles, Zebo Peng, Sudipta Chattopadhyay, Lejla Batina. SPARTA: A Scheduling Policy for Thwarting Differential Power Analysis Attacks, Asia and South Pacific Design Automation Conference (ASPDAC), Macao SAR, China, January $2016\left(\left[\mathrm{JEP}^{+} 16\right]\right)$

The following publications are not directly covered by this thesis, but are generally related to the design of secure real-time systems:

- Wei Jiang, Ke Jiang, and Yue Ma. Resource Allocation of Security-Critical Tasks With Statistically Guaranteed Energy Constraint. International Conference on Embedded 
and Real-Time Computing Systems and Applications (RTCSA), Seoul, Korea, August 2012 ([JJM12])

- Xia Zhang, Jinyu Zhan, Wei Jiang, Yue Ma, and Ke Jiang. Design Optimization of Energy- And Security Critical Distributed Real-Time Embedded Systems. International Parallel and Distributed Processing Symposium Workshops (IPDPSW), Boston, USA, May $2013\left(\left[\mathrm{ZZJ}^{+} 13 \mathrm{a}\right]\right)$

- Xia Zhang, Jinyu Zhan, Wei Jiang, Yue Ma, and Ke Jiang. Design Optimization of Security- Sensitive Mixed-Criticality Real-Time Embedded Systems. Workshop on Real-Time Mixed Criticality Systems (ReTiMiCS), Taipei, Taiwan, August $2013\left(\left[\mathrm{ZZJ}^{+}\right.\right.$13b] $)$

- Ke Jiang, Petru Eles, Zebo Peng, and Wei Jiang. PowerAware Design of Secure Multi-Mode Real-Time Embedded Systems with FPGA Co-Processors. International Conference on Real-Time Networks and Systems (RTNS), Sophia Antipolis, France, October $2013\left(\left[\mathrm{JLE}^{+} 13\right]\right)$

- Wei Jiang, Ke Jiang, and Yue Ma. Energy Aware RealTime Scheduling Policy with Guaranteed Security Protection. Asia and South Pacific Design Automation Conference (ASPDAC), SunTec, Singapore, January 2014 ([JJM14])

- Ke Jiang, Lejla Batina, Petru Eles, and Zebo Peng. The Influence of Real-Time Scheduling On Differential Power Analysis Attacks. TRUDEVICE workshop: (Co-located with DATE15), Grenoble, Franch, March 2015 ([JBEP15])

- Wei Jiang, Ke Jiang, Xia Zhang, and Yue Ma. Energy Optimization of Security-Critical Real-Time Applications With Guaranteed Security Protection. Journal of Systems Architecture (JSA), 2015 ([JJZM15])

- Liang Wen, Wei Jiang, Ke Jiang, Xia Zhang, Xiong Pan, and Keran Zhou. Detecting Fault Injection Attacks on Embedded Real-Time Applications: A System-Level Per- 
spective. IEEE International Conference on High Performance Computing and Communications (HPCC), New York, USA, August $2015\left(\left[\mathrm{WJJ}^{+} 15\right]\right)$

- Xiong Pan, Wei Jiang, Ke Jiang, Liang Wen, and Qi Dong. Energy Optimization of Stochastic Applications with Statistical Guarantees of Deadline and Reliability. Asia and South Pacific Design Automation Conference (ASPDAC), Macau SAR, China, January $2016\left(\right.$ PJJ $\left.\left.^{+} 16\right]\right)$

\subsection{Thesis Organization}

This thesis is organized in seven chapters. In Chapter 2, we shall discuss the general background and current status of research related to embedded system design and embedded system security. In Chapter 3, we shall present some preliminaries related to the hardware architecture and power models and also the involved security definitions. Our contributions to achieving secure distributed RTESs will be presented in Chapter 4. In Chapter 5, we shall elaborate on an overall design framework for secure multi-mode RTESs considering four design objectives. In Chapter 6, we shall discuss the influence of real-time schedulers on system robustness against SCAs, and present a novel scheduling policy for thwarting SCAs. Chapter $\mathbf{7}$ concludes the thesis, and points out possible future research directions in the context of secure RTES designs. 


\section{Chapter 2}

\section{Background and Related Work}

$\mathrm{I}^{\mathrm{N}}$ this chapter, we shall discuss the background of this thesis, and Section 2.1 outlines a general embedded system design flow, and highlights the stages that are particularly relevant from the point of view of this thesis. Section 2.2 presents the common requirements typical for embedded system designs, i.e., energy efficiency, timeliness, and multi-mode operation, and the related work along these directions. Section 2.3 discusses existing approaches for designing secure embedded systems.

\subsection{Embedded System Design}

Embedded systems (ESs) are "information processing systems embedded into enclosing products" [Mar11]. Classical examples of embedded system applications include vehicles, consumer electronics, and airplanes. For example, it is common to find tens of microprocessors (Electronic Control Units or ECUs) in modern vehicles connected by a communication infrastructure, e.g., controller area network (CAN) [CAN91] or Flexray [Fle05]. Common requirements shared among various ESs are real-time guarantees, energy constraints, and cost efficiency. Recently, the concept of cyber-physical system (CPS) [Lee08, 


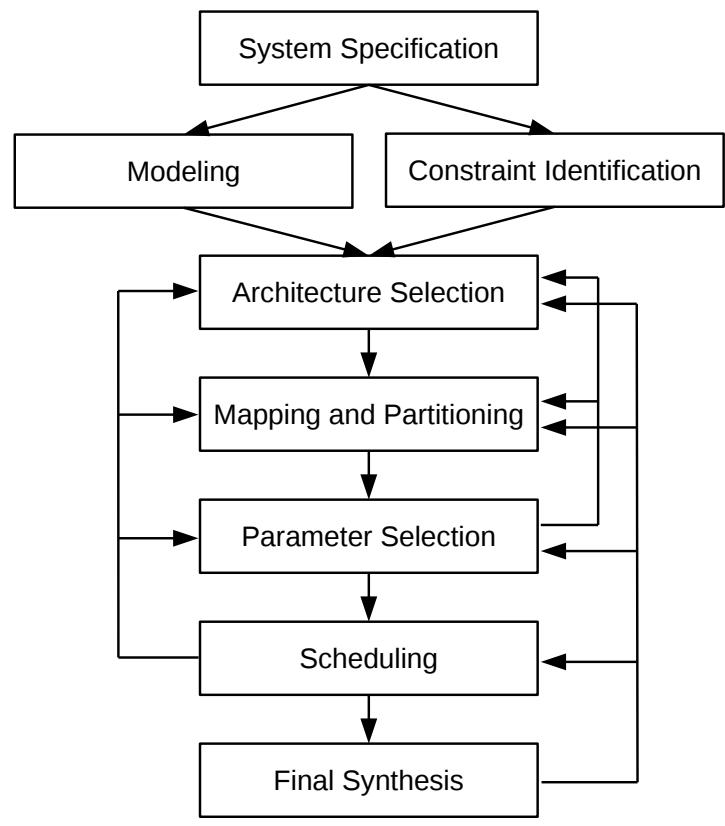

Figure 2.1: System design flow

RLSS10] has been introduced, emphasizing the tight interaction between embedded systems and the physical environment. The systemlevel design of ESs (and CPSs) is usually carried out in several stages as depicted in Figure 2.1.

In the System Specification stage, details of the system functionality are described. The designer concretely specifies the jobs the system will handle and the general requirements the system must satisfy. This is the very first step of the design process and provides the foundation for later stages.

In the Modeling step, the designer divides the overall system functionality into smaller modules depending on various aspects. For example, the modules can be abstracted based on the associated hardware units for achieving higher parallelism, or depending on different functionalities. In this thesis, the system is modeled as a set of computation tasks, each of which may represent a basic software block or an independent application, depending on the level of abstraction. 
At Constraint Identification, the set of constraints imposed on the system are identified, e.g., the deadline of executions, energy limitations, and security requirements. These constraints are used to drive the decision makings in later stages.

During Architecture Selection, the designer decides on a concrete hardware platform for undertaking the tasks. Several parameters must be considered in the selection, e.g., the organization of the system (distributed or centralized) and cost constraints (more powerful or less powerful processors, or whether to use reconfigurable hardware for accelerations). In the beginning, the architecture is chosen based on estimations and needs to be revisited with feedbacks from later stages to optimize the performance/cost ratio and satisfy the given design constraints.

During Mapping and Partitioning [EPKD97, DLJ99], designer allocates the tasks on available processing units, either in software on microprocessors or to hardware modules. The objective is to pursue the best system performance, e.g., minimizing the end-to-end delay or maximizing delivered service quality.

In the Parameter Selection stage, the system execution parameters, e.g., extra amount of executions and operation frequencies are tuned for achieving higher performance or efficiency. The selection is driven by one or multiple objective functions reflecting concrete design expectations under given constraints [IPEP05, IPEP08]. Usually, such problems of RTESs are of huge complexities, to which it is impossible to find the optimal solutions. Therefore, good heuristic approaches must be deployed to solve the problem efficiently [DPAM02]. In addition, this stage is often integrated with the next stage, i.e., Scheduling to reach globally better solutions. If no satisfying solution is found, then the designer must revisit the decisions from the previous stages, and find potential improvements, e.g., use more powerful processors or derive a more efficient mapping (indicated by the backwards arrows from the Parameter Selection stage in Figure 2.1).

In the Scheduling stage, the tasks mapped on the computational resources will be scheduled to meet the imposed real-time constraints. A multitude of techniques have been proposed in literature for task scheduling. If the task executions can be interrupted, the schedul- 
ing policy is called preemptive scheduling [BMR90]. Otherwise, nonpreemptive scheduling is used $\left[\mathrm{GYG}^{+} 08\right]$. Static cyclic scheduling implies the off-line generation of a fixed schedule table that will be followed at run-time to order task executions [XP00, DK08]. Priority based scheduling policies select and execute the task with the highest priority, when there are requests from multiple tasks. Depending on whether the priority of a task is constant or not, priority based scheduling can be further divided into two groups, static priority scheduling and dynamic priority scheduling [LL73, But11]. Quasi-static scheduling tries to combine the advantages of the previous approaches, and prepares a set of possible alternatives off-line that can be used at run-time [CEP04, WMWZ12] for pursuing higher performance. It is also possible that the deadline constraints cannot be satisfied in any solutions under the current setup. Then the designer must revise the previous decisions, e.g., architecture selection (indicated by the arrows from the Scheduling stage in Figure 2.1).

In the Final Synthesis stage, the solution obtained from the Scheduling stage is reviewed. If all the requirements and constraints are met, the design will proceed with the final synthesis of the low level hardware/software implementations. Otherwise, one or several steps have to be revisited.

This thesis mainly focuses on the Mapping and Partitioning, Parameter Selection, and Scheduling stages of the overall design flow.

\subsection{Design Requirements}

\subsubsection{Energy Efficiency}

Embedded systems are very often battery-driven or with limited energy budget. Hence, energy efficiency is a must in modern embedded system design. This can be achieved by different methods. A very popular technique for reducing energy consumption is via utilizing the dynamic voltage and frequency scaling technology (DVFS) [BB00, PS01]. The authors of [BAEP09] proposed a thermal aware DVFS approach for reducing system energy consumption which considered the temperature influence on frequency and, consequently, on 
energy. The authors of [ML09] proposed a warp processor architecture that leverages DVFS to reduce the power consumption. The authors of [PJPM14] described an integrated power manager that exploited the DVFS capabilities of embedded CPU and GPU together. This is related to the Parameter Selection stage in Figure 2.1. The authors of [HCK06] presented an energy-efficient task scheduling approach for heterogeneous architecture with FPGA co-processors, in which the on-board FPGA resources were used both for performance enhancing and energy saving purposes.

\subsubsection{Timeliness}

Real-time embedded systems (RTESs) are those systems in which not only producing correct results is important, but the timeliness of result delivery is also critical. That is, late delivery of a correct result may cause severe system errors. An example of RTES is the brakeby-wire system. When the driver signals a request of braking, the car must react in due time by adjusting the brakes accordingly. This requires efficient mechanisms to guarantee the timeliness of result deliveries, and is mainly related to the Scheduling stage.

The synthesis of RTESs and analysis of real-time properties have been extensively studied by the research community. The authors of [SSL89] presented the Sporadic Server algorithm for scheduling aperiodic independent tasks, and showed the improvement of response times for the tasks with soft-deadlines as well as the guarantees of deadline satisfactions of tasks with hard-deadlines. The authors of [BBMSS10] presented the schedulability tests for global EDF and deadline monotonic policies on multiprocessor platforms. The authors of [AEP15] studied the influence of jitter on stability of real-time control applications, and presented an on-line scheduling policy to limit task response time variations, and thus, to guarantee stability of the control application.

\subsubsection{Multi-Mode Operation}

Embedded systems today are very often expected to function under a dynamically changing load. This leads to the concept of multi-mode 
systems [OH02] which are exposed to dynamic loads with the number and functionality of active tasks changing during run-time. The uncertainty about the execution mode that the system is going to run in at run-time leads to huge design complexity for delivering the best performance in all possible cases. That is, the number of potential combinations of tasks that are to be processed by the system can be very large, which makes designing multi-mode RTESs an extremely complex problem. This problem mainly affect the Parameter Selection and the Scheduling stages.

Design of multi-mode systems has been well studied. In [MZ05], the energy-efficiency problem of multi-mode distributed RTESs was approached by distributing available slack execution times. The authors of [SAHE05] presented an energy minimization framework for multi-mode distributed RTESs considering the mode execution properties. The authors of [SEPC09] proposed a flexible synthesis approach for multi-mode embedded control systems that can be tuned for better control quality or faster design time. The authors of [SCT10] presented a power-aware task mapping approach for multi-mode multiprocessor SoCs (system on chips). The presented techniques precompute and store a set of mapping strategies off-line, and then online decide the most power efficient mapping for a newly arriving task using the pre-stored knowledge. The authors of [WAST12] presented an on-line framework for placing multi-mode streaming applications onto partially reconfigurable FPGAs such that the dynamic reconfiguration overhead is minimized. The authors of [LEP15] presented an on-line resource manager for real-time multi-mode applications running on heterogeneous platforms such that the global energy consumption of the system is minimized.

\subsection{Secure Embedded Systems Design}

As embedded systems are now expected to cope with new applications and challenges, requirements on security characteristics are emerging in the design processes beside the aforementioned aspects. In this section, we shall discuss about the new design requirements related to security. The authors of [RRKH04] outlined the global design chal- 
lenges of secure embedded systems. In addition, the adoption of new communication interfaces, e.g., Wi-Fi, that enable tighter interactions between RTESs and the surrounding environment, dramatically increase potential security threats [ZM05, CAS08]. With the trend towards more and more communication demands, sensitive information exchanged among nodes inside a system or with external peers and service centers has been exposed to attackers, which leads to the need for sound cryptographic protections and robust side-channel attack resistance.

\subsubsection{Cryptography and Other Security Services}

Previous work on ES communication mainly focused on the protocol and application issues from the safety and reliability perspectives [CS07], while the potential security risks have been seriously overlooked. The authors of [WWP06] stated that the internal communication inside modern vehicles is completely unprotected, which is, though, tightly related to safety and privacy. The authors of $\left[\mathrm{KCR}^{+} 10\right]$ demonstrated the possibility of hacking into an automotive electronics system remotely and reading out various information of the car, sometimes quite easily. The same research group successfully mounted another set of attacks purely remotely without any physical accesses $\left[\mathrm{CMK}^{+} 11\right]$. Recently, the news that two hackers attacked a vehicle remotely, e.g., turning on/off the fans and even shutting down the engine, caught huge media coverage [Gre15]. All these works urged the need for security protections in automobiles, and more generally, in all critical RTESs.

A RTES can be of different types and architectures from a component unit of a larger system, e.g., the control system of a unmanned aerial vehicle (UAV) or an end node in a large network, to a system that is distributed over a certain area. However, regardless of the architecture, confidentiality of the communication is arguably the most important component out of the security concepts in the context of RTESs. This is because the messages exchanged via the communication module or infrastructure can contain sensitive information related to the user's privacy or critical system status of the controlled 
plant. Such communication needs to be protected against malicious eavesdropping [ILW06, HKD11]. However, the RTES communication protocols usually do not come with any security protections, e.g., for confidentiality or integrity [WWP04]. This makes security protection a mission that the designer must take care of on the application level.

There are works discussing the communication security of distributed embedded systems [GGS04, PP05, RH07] focusing on the vehicle to vehicle (V2V) and vehicle to infrastructure (V2I) communication. In [WWP06], the authors presented feasible attacks and an overall cryptographic architecture for automotive communication networks. However, the actual resource and timing constraints imposed in the systems were not touched. In [HKD11], the authors described four practically implemented attack scenarios, and brought forward the necessity of applying cryptography to protect the internal bus communication. The authors of [CAYM15] discussed the vulnerabilities and possible attacks to the CAN protocol which is one of the most applied bus protocol in modern vehicles, and also listed several high-level solutions to overcome the security drawbacks. The authors of $\left[\mathrm{MSL}^{+} 15\right]$ presented a lightweight authentication framework for automotive communication networks, that allows secure distribution of secret keys without pre-shared secrets.

The authors of [SV03] presented a cryptographic co-processor architecture for efficient message encryptions in embedded systems. A hardware/software co-design technique to protect embedded systems against buffer overflow attacks was presented in [SXZ ${ }^{+}$06]. In [PP08], the authors presented an automatic hardware-software design flow for detecting code injection attacks in multiprocessor SoCs. The authors of [WDL14] presented a model-based design technique considering potential security attacks in the design procedure. However, one of the fundamental design requirements of ESs, namely the real-time aspect, was not present in these works.

There are only a limited number of approaches that try to deliver sound security protection considering the actual timing requirements. In [XQ07], the authors presented an on-line scheduling policy that distributes free time slacks to enhance security services while guaranteeing the real-time constraints. However, the authors solely 
considered the security services as the only optimization goal, and ignored the other design requirements that may also be imposed in the system, e.g., energy efficiency and quality of service. Moreover, due to the rather simple slack distribution mechanism, the presented scheduler may struggle to find good solutions purely at run-time. The authors of $\left[\mathrm{LXY}^{+} 09\right]$ proposed two off-line techniques to optimize the security protections of the real-time systems running on monoprocessors and then rely on EDF to schedule the tasks on-line. However, neither of the techniques is capable to be applied in the systems in which the set or the nature of tasks changes at run-time, because of the high optimization overhead. In addition, neither of these two works touched the problem of protecting RTESs against side-channel attacks. The authors of $\left[\mathrm{YMC}^{+} 13\right]$ presented a multicore-based architecture for detecting potential intrusions to RTESs based on inherent timing properties of execution profiles. However, the multi-mode and communication confidentiality aspects were not discussed.

\subsubsection{Side-Channel Attacks and Protections}

The fundamental step towards security in RTESs is to carry out cryptography and other dedicated protection services. The communication confidentiality particularly requires cryptographic protections, i.e., via message encryption and decryption using cryptographic algorithms. However, the implementations of such algorithms may become the target of attacks. For example, the side-channel attacks (SCAs), which are the most dangerous type of attacks that target the implementations of cryptographic algorithms (including AES), have raised severe alarms regarding embedded system security. In [KJJ99], Paul Kocher et al. presented the so-called differential power analysis attack (DPA), one type of SCA, that has become one of the most efficient attack schemes targeting cipher implementations on embedded platforms. For example, the works of [OGOP04] and [SÖP04] presented the first DPA attack on ASIC (application-specific integrated circuit) and FPGA implementation of AES, respectively. The authors of [MOP07] thoroughly discussed the DPA attacks on software implementations of AES. 
There have been numerous works that try to protect the systems against DPA attacks. Random delay insertion (RDI) is a common approach for counteracting DPAs. For example, the authors of [CK09] presented a software approach for generating random delays, which can be used to resist DPAs. The authors of $\left[\mathrm{BVR}^{+} 13\right]$ presented an automated hardware design methodology that inserts random jitters for thwarting DPAs. Another popular approach is via randomizing the data being processed on the device, known as masking, to make the power consumption independent of the processed data [Geb06, CB08]. Architectural modification that alters the power trace is also a widely used technique for preventing DPAs on cipher implementations [MD11, MD12]. However, none of these works can be easily applied in RTESs, since the timing constraints and energy limitations may be violated in the resource limited environments, or changes to the underlying hardware are expected. 


\section{Chapter 3}

\section{Preliminaries}

$\mathrm{T}^{\mathrm{N}}$ this chapter, we shall present the preliminaries related to the 1 architectures and power models we assume throughout the thesis. We shall also define the security metrics we use in this thesis. Section 3.1 presents the overall hardware architecture and power model we consider. Section 3.2 elaborates on the involved security metrics used in this thesis.

\subsection{Hardware Architecture and Power Model}

\subsubsection{Hardware Architecture}

Figure 3.1 illustrates an overall hardware structure of a RTES, that is composed of several subsystems, i.e., a subsystem running on a distributed platform, e.g., Figure 3.1 (a), and two subsystems on two independent computational units, e.g., Figure 3.1 (b). The subsystems are connected via a central gateway, which handles the communication among different subsystems, and also takes charge of external communication with the outside world.

The subsystem illustrated in Figure 3.1 (a) is referred to as a distributed RTES, of which the hardware architecture consists of a set of computational units, i.e., embedded processors, electronic control units, and FPGA (field-programmable gate arrays) devices, connected by a communication bus, e.g., CAN [CAN91]. The computational units take care of all processing requests, e.g., processing raw sen- 


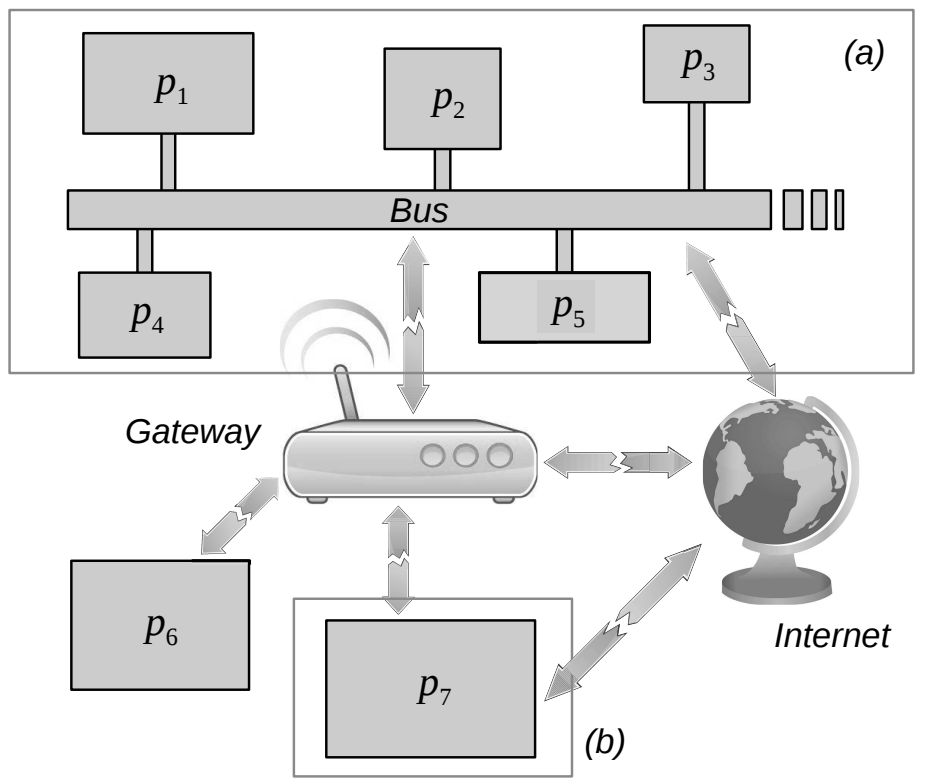

Figure 3.1: An overall hardware architecture

sor data and control signals. While, the message exchanges between different computational units are accomplished by transmitting messages over the underlying bus infrastructure.

For pursuing higher efficiency and performance, reconfigurable hardware devices, especially FPGAs, have been extensively utilized in embedded system designs [HV08, ZSJ09, ZSJ10]. In this thesis, we consider that the FPGA co-processors (if present in the system) support static reconfiguration and possibly also partial dynamic reconfiguration (PDR). If PDR is supported, part of the FPGA area can be dynamically reconfigured while the rest of the FPGA continues to process. In fact, modern FPGA families, like the Xilinx Virtex or Altera Stratix, provide efficient partial dynamic reconfiguration support. This offers great flexibility, allowing customization of the hardware platform according to different system requirements. One scenario often employed for current reconfigurable platforms is that the FPGA is partitioned into a static and a PDR region. The static region hosts a microprocessor, a reconfiguration controller (which takes 
care of reconfiguring the PDR region), and, potentially, other peripheral modules that need not change at run-time. The PDR region is organized as reconfigurable slots (composed of heterogeneous configurable tiles), where hardware modules can be reconfigured at run-time $\left[\mathrm{KLH}^{+} 11\right]$.

The other type of subsystems shown in Figure 3.1 can be an independent system or an end component of a bigger system. Such a unit consists of a DVFS-enabled microprocessor connected with a set of peripherals, e.g., sensors and actuators, and communication modules, via which the unit interacts with other peers or service centers (over wire or wirelessly). The supply voltage (and implicitly the frequency) of the processor can be selected from a discrete set, depending on actual operational requirements.

\subsubsection{Power Model}

In this section, we present the models we use for estimating the power consumption of the processors. The power consumption of a processor (designed with CMOS technology) consists of several parts: dynamic power $\left(\right.$ Pow $\left.^{\text {Dyn }}\right)$, static power $\left(\right.$ Pow $\left.^{\text {Stat }}\right)$, inherent power $\left(\right.$ Pow $\left.^{\text {On }}\right)$ [MFMB02], and short circuit power. Short circuit power consumption occurs only during signal transitions, and is negligible [Vee84]. Pow ${ }^{\text {On }}$ represents the inherent power consumption incurred by keeping the processor on, and has a constant value. The dynamic power consumed by the processor can be calculated as

$$
\text { Pow }^{\text {Dyn }}=C_{e f f} V_{d d}^{2} f,
$$

where $C_{e f f}, V_{d d}$ and $f$ denote the effective switching capacitance due to computations, the supply voltage, and clock frequency of the processor, respectively. The dependency of the operating frequency on supply voltage [MFMB02] is given by

$$
f=\frac{\left(\left(K_{4}+1\right) V_{d d}+K_{5} V_{b s}-v_{t h 1}\right)^{\alpha}}{K_{6} L_{d}},
$$

where $K_{4}, K_{5}, K_{6}$ and $v_{t h 1}$ are technology dependent coefficients, $L_{d}$ is the logic depth, and $\alpha$ is a measure of velocity saturation. In this 
thesis, we assume that the processor can run at discrete designated voltages, and, consequently, at the corresponding discrete frequencies. Dynamic power is consumed only when the processor is active, i.e., executing tasks. Static power does not depend on switching activity, and is consumed due to leakage current, which is mainly a combination of sub-threshold conduction $\left(I_{\text {sub }}\right)$ and reverse bias junction current $\left(I_{j u}\right)$ [MFMB02]. The static power (consumed both when the processor is active and idle), is given by

$$
P o w^{\text {Stat }}=L_{g}\left(V_{d d} I_{s u b}+\left|V_{b s}\right| I_{j u}\right),
$$

where, $L_{g}$ is the amount of logic gates in the circuit, and $V_{b s}$ is the voltage applied between the body and the source of a transistor. As shown in [MFMB02], the sub-threshold leakage current can be approximated with the following expression

$$
I_{s u b} \approx K_{1} e^{K_{2} V_{d d}} e^{K_{3} V_{b s}},
$$

where $K_{1}, K_{2}$ and $K_{3}$ are constant fitting technology dependent parameters.

\subsection{Security in Embedded Systems}

In this thesis, we focus on two representative security design objectives that are particularly important in the context of embedded systems, namely, confidentiality and intrusion detection. In order to achieve these two goals, we must implement corresponding protection mechanisms, that are cryptography for delivering confidentiality and intrusion detection applications for detecting potential intrusions. Note that, the confidentiality protection strength of cryptographic algorithms lies in two aspects with respect to two different attack methods. We shall elaborate on this in more details in the next section.

\subsubsection{Confidentiality}

Confidentiality is usually of central importance among the key components of embedded system security. In this thesis, we shall focus 
on achieving confidentiality for the communication. While, the confidentiality aspects of the data storage, e.g., how to securely save and keep critical data in memory, is not in the scope of this thesis.

The fundamental step towards communication confidentiality is to apply cryptography. In order to protect the confidentiality of the communication, we must carry out encryption/decryption (E/D) on the exchanged messages. However, this comes with extra computational overhead, which is a problem for the designers, since embedded systems very often have limited computational capacity and have to function under stringent timing constraints. There are three main approaches in cryptography: public-key cryptography, symmetric-key cryptography, and cryptographic hash functions. In public-key cryptosystems, different but related keys are used, including a public key and a private key. They have mainly been developed based on the computational complexity of certain hard mathematical problems, e.g., integer factorization, and are relatively costly in computational demand, compared to most symmetric key algorithms with equivalent security level. This has limited their use in resource constrained environments like embedded systems for encryption purposes.

In symmetric-key cryptography, the same key (or trivially related keys) is used for both encryption and decryption. The key represents a shared secret between two or multiple parties that have access to the confidential information. Such crypto-algorithms, e.g., AES [DR02] and RC6 [RRSY98], have been designed to be highly efficient, even on embedded microprocessors. In resource constrained systems, publickey algorithms can be used for occasionally exchanging secret keys for symmetric-key algorithms which will perform the actual message encryption and decryption. Cryptographic hash functions are used for verifying the integrity of a message or the identity of the sender. By this, the convenience and efficiency of the three different cryptosystems are combined. In this thesis, we shall concentrate on maintaining confidentiality of the communication by utilizing arguably the most widely used branch of symmetric cryptography, the iterated block ciphers. 


\subsubsection{Iterated Block Ciphers}

Iterated block ciphers (IBCs) are one type of symmetric-key cryptography and are widely adopted for protecting information confidentiality. IBCs are constructed by applying a function repeatedly in order to provide better information confusion and diffusion as the number of rounds increases. They are particularly suitable for embedded systems [PP10] because of their properties of high throughput, implementation simplicity, and sound protection strength. In this thesis, we focus on using two representative IBCs, i.e., the Advanced Encryption Standard (AES or Rijndael) [DR02] and the very flexible RC6 block cipher [RRSY98], for encrypting and decrypting messages under different design requirements. However, the techniques presented in this thesis are general enough to be also applied on other IBCs.

The execution time (ET) (nominal time with respect to a given execution setup, i.e., platform and frequency) of an E/D task of an IBC on a message $m_{i}$ is growing linearly with the number of rounds. In other words, the more rounds are used for encryption and decryption, the longer execution time the procedure will take, but the closer the output is to a random bitstream, making it more secure. For correct message transmission, the same number of rounds must be used by an E/D process pair. Since the E/D procedures of a chosen IBC on message $m_{i}$ are similar but inverse to each other, they have roughly the same nominal ET calculated as follows,

$$
\mathcal{E}_{E D}=w_{E D}+r_{E D} * x_{i}
$$

where, $w_{E D}$ represents the constant nominal ET of the chosen IBC for doing the pre-/post-whitening on the encryption and decryption tasks, representing the initialization and finalization operations of the algorithm. The coefficient $r_{E D}$ represents the nominal ET for doing one round of E/D. Variable $x_{i}$ is the number of rounds used by the chosen cipher variant $C_{i}$ for $m_{i}$. However, the actual ETs for the E/D tasks depend on the computational resources they are mapped to and the frequencies they are executed at. We shall come back to this in later chapters. 


\subsubsection{Protection Strength of IBC}

If the only information the attacker has access to is the transmitted data (for example, she does not have physical access to the computational units, or the whole computational system including the power supply is protected with tamper-resistance technology [RRC04]), then she can mount attacks only based on the captured messages. In addition, we assume a strong attacker who also can feed the cryptosystems with arbitrary plaintexts. Under such a scenario, the attacker can do cryptanalysis attacks on the IBC algorithm, trying to recover secret information based on the theoretical weaknesses of the algorithm.

A large number of plaintexts and their corresponding encrypted versions (the cipher texts) generated from the target system are required in order to do a successful attack [BS91]. The efforts needed to gather such required information grow exponentially as more rounds are used, because, as mentioned in the previous section, closer-torandom cipher texts are produced. From now on, we shall refer to a chosen implementation of an IBC with $x$ rounds as one of its variant. Due to the actual timing and resource constraints, as well as considering the current security threats present in the RTESs, we may have to sacrifice certain protection strength, i.e., using variants with less rounds of the chosen IBC for faster encryption or decryption ${ }^{1}$. Then the question is how good protection an IBC variant delivers with respect to the chosen number of rounds.

In order to quantify the protection strength of an IBC and compare the strengths of different IBCs or IBC variants, we capture the IBC strength as the logarithm of the number of plaintext-cipher pairs needed to break the algorithm by the best known cryptanalysis attack. We have conducted extensive studies on cryptanalysis attacks on four representative IBCs, i.e. RC5 [BK98], RC6 [KM01, HKHF11], AES [ZWF07, LDKK08] and Blowfish [Vau96]. In the end, we have chosen seven variants of the RC6 algorithm which enjoy the highest rate of protection strength to encryption speed ratio, among all investigated IBCs. RC6 is simple and flexible while providing sound security

\footnotetext{
${ }^{1}$ Remember that the encryption and decryption time is linear to the number of rounds.
} 
Table 3.1: Protection strength and encryption time of different RC6 variants

\begin{tabular}{|l|c|c|c|c|c|c|c|}
\hline Variant & RC6-4 & RC6-6 & RC6-8 & RC6-10 & RC6-12 & RC6-14 & RC6-16 \\
\hline Strength & 29 & 45 & 61 & 78 & 94 & 110 & 118 \\
\hline Time & 23 & 31 & 38 & 46 & 54 & 62 & 70 \\
\hline
\end{tabular}

protection $\left[\mathrm{NBB}^{+} 00\right]$, if the design parameters are carefully decided.

The results of our study on the seven chosen RC6 variants are listed in Table 3.1, in which RC6- $x$ means $x$ rounds are carried out for this variant of RC6. Half of the data is encrypted in one round in RC6, so two rounds are considered as the smallest unit for doing encryption and decryption using RC6 in the rest of this thesis. The first row in Table 3.1 presents the referred RC6 variants indexed by the number of applied E/D rounds $(4,6,8$, etc). The second and third rows list the corresponding protection strength (logarithm based) and execution time ( $\mu s)$ per block (16 bytes $)^{2}$, respectively. The encryption and decryption times of a RC6 variant are very similar, so we assume them equal for simplicity of the presentation (as shown in Eq 3.4). The variants of RC6 with more than 16 rounds of E/Ds are omitted, since they are considered to be fully secure against cryptanalyses $\left[\mathrm{NBB}^{+} 00\right]$. It is worth mentioning that our proposed design framework is general enough to be applied to other quantification methods and cryptographic algorithms or variants too, if similar strength/time trade-offs can be derived.

\subsubsection{Side-Channel Attacks on IBC}

If the attacker has physical access to the hardware, then she can do more powerful attacks to the target device. We assume that the actual computational units, e.g., the processor and memory, are tamperresistant [RRC04]. That is, the attacker cannot directly read the sensitive information from the memory or registers, or control the

\footnotetext{
${ }^{2}$ Estimated from ANSI C implementation of RC6 on a processor running at the frequency of $7.2 \mathrm{MHz}$ [RRSY98].
} 
processor operations. As in the previous section, we also assume a strong attacker who can feed the system with arbitrary plaintext, e.g., by changing the data from the sensors. In addition, we also assume that she can accurately measure the power consumption of the microprocessor. As the microprocessor is tamper-resistant, she cannot change the task schedule, or directly read out the secret key(s), but instead, she can mount attacks based on the side-channel information that she can measure (e.g., the power consumption) aiming to find secret information of the system.

As mentioned in Section 2.3.2, DPA attacks are arguably the most efficient attack scheme on IBC implementations. Therefore, we focus on DPA attacks on AES in this thesis. The DPA attacks try to reveal the secret key $K$ used by AES at the granularity of subkey ${ }^{3}$. The procedure is, in brief, as follows. The attacker first identifies a fraction in an AES round that is a function of a given text and an 8-bit subkey $k$. This fraction is referred to as a leakage point $L P_{k}$. Of all the AES encryption (or decryption) operations with the same AES secret key $K$, the same subkey $k$ is used to operate on different input texts using the same function at $L P_{k}$. Therefore, there exists a certain relation among all the measured power consumptions at occurrences of $L P_{k}$. After identifying $L P_{k}$, the attacker feeds the AES process with chosen plaintexts, and measures the power of the processor. Then, based on a timespan $T S$ that the attacker defines, she divides the whole obtained power trace (of $G$ time units) into $S=G / T S$ samples. In fact, based on our observations from a large number of experimental evaluations, choosing $T S=\mathcal{P}(\mathcal{P}$ is the period of the AES task $c e / c d$, which depends on the period of its parent task associated with the corresponding message.) gives the best alignment among all the possible values of $T S$. This is to the most advantage of the attacker, and also the worst case from the designer's perspective. So we assume that the attacker knows $\mathcal{P}$ to reserve sufficient security margin. Then the attacker organizes the samples into a $2 \mathrm{D}$ matrix $P=[i-j](i=1, \ldots, S ; j=1, \ldots, V)$ with size $S * V$, in which $V=\mathcal{P} \times F . F$ is the sampling frequency of the attack equipment.

\footnotetext{
${ }^{3}$ The definition and how to obtain the AES subkeys can be found in [DR02].
} 


\begin{tabular}{|c||c|c|c|}
\hline$P_{1,1}$ & $P_{1,2}$ & $\ldots$ & $P_{1, I}$ \\
\hline$P_{2,1}$ & $P_{2,2}$ & $\ldots$ & $P_{2, I}$ \\
\hline$\ldots$ & $\ldots$ & $\ldots$ & $\ldots$ \\
\hline$P_{S, 1}$ & $P_{S, 2}$ & $\ldots$ & $P_{S, I}$ \\
\hline
\end{tabular}

\begin{tabular}{|c|c|c|c|}
\hline$H_{1,1}$ & $H_{1,2}$ & $\ldots$ & $H_{1, K}$ \\
\hline$H_{2,1}$ & $H_{2,2}$ & $\ldots$ & $H_{2, K}$ \\
\hline$\ldots$ & $\ldots$ & $\ldots$ & $\ldots$ \\
\hline$H_{S, 1}$ & $H_{S, 2}$ & $\ldots$ & $H_{S, K}$ \\
\hline
\end{tabular}

Pearson's

correlation

\begin{tabular}{|c|c|c|c|}
\hline$\rho_{1,1}$ & $\rho_{\AA, 2}$ & $\ldots$ & $\rho_{1, I}$ \\
\hline$\rho_{2,1}$ & $\rho_{2,2}$ & $\ldots$ & $\rho_{2, I}$ \\
\hline$\ldots$ & $\ldots$ & $\ldots$ & $\ldots$ \\
\hline$\rho_{K, 1}$ & $\rho_{K, 2}$ & $\ldots$ & $\rho_{K, I}$ \\
\hline
\end{tabular}

Figure 3.2: Calculate correlations between power measurements and hypotheses

Thus, $V$ is the number of obtained power values within $\mathcal{P}$. Each $P_{x, y}$ denotes an actual measured power value of sample $x$ at relative time point $y$ (with respect to $F$ ).

The next step is to produce the hypotheses regarding the processor power at leakage point $L P_{k}$. Since there are only $2^{8}=256$ different possibilities of subkey $k$, the attacker can enumerate all the possible 256 values on all the plaintexts she used, and derive another 2D matrix $H=[i-j](i=1, \ldots, S ; j=1, \ldots, 256)$, each of which is a hypothetical power value of the corresponding plaintext-subkey pair, and is calculated depending on her knowledge about the underlying hardware.

The last step is to find correlations between the actual measured power of the processor and the attacker's hypothetical power on each column $P_{i}$ of $P$ and $H_{j}$ of $H$ by calculating, for example, the Pearson correlation coefficient $\rho_{i j}$ (as shown in Figure 3.2). Column $P_{i}$ and $H_{j}$ has high correlation if $\rho_{i j}$ is high. And the highest value $\rho_{\max }=\rho_{x y}$ reveals that $k_{y}$ was the real subkey used at relative time $t_{x}$. Then, the attacker tries to recover the whole secret key $K$ by going through all the subkeys, or until it is trivial to mount a brute-force attack on 
the rest of the key bits.

Then the question is how difficult it is to retrieve the secret key $K$ from the attacker's perspective, or how robust $K$ is from the designer's point of view. Assuming that the highest correlation between two columns of $P$ and $H$ is $\rho_{\max }$, the attacker aims to get a value of $\rho_{\max }$ which stands out among all the values $\rho_{i j}$. Due to various reasons, e.g., clock drifting, the occurrences of leakage point $L P_{k}$ with respect to subkey $k$ may happen at different times in different samples, thus, leading to lower values of $\rho_{\max }$. If $\forall \rho: \rho \approx 0$, then there is no dominating $\rho_{\max }$, that is, there is no clear correlation between any column of $P$ and $H$. This is the optimal case in terms of robustness against DPA of the AES implementation, meaning that the attacker needs infinite amount of samples to observe a high $\rho_{\max }$. Let us denote the moment of time when $L P_{k}$ happens with the highest probability among all the samples as $\hat{t}$. The corresponding column of $P_{\hat{t}}$ will have the highest correlation with the column $H_{s k_{i j}}$ of $H$ which is the actual subkey used in the leakage point. The probability that the leakage point occurs at $\hat{t}$ is captured as $\hat{p}$.

We assume that the processor execution is noise-free, that is the signal-to-noise ratio (SNR) is 0 , to reserve sufficient security margin. The number of samples defining the strength of the secret key against DPAs can be calculated with Equation 4.44 from [MOP07] as follows,

$$
\mathcal{N}=3+8 \cdot \frac{z_{1-\alpha}^{2}}{\ln ^{2}\left(\frac{1+\rho_{\max }}{1-\rho_{\max }}\right)},
$$

where $\alpha$ and $1-\alpha$ are known as the error probability and confidence interval of detecting a significant $\rho_{\max }$ from all the correlation values between the columns (see Figure 3.2). $z_{1-\alpha}$ is the quantile of standard normal distribution that determines the distance between the distribution of $\rho=0$ and $\rho=\rho_{\max }$, and is calculated as follows

$$
z_{1-\alpha}=\sqrt{2} \cdot \operatorname{erf}^{-1}(1-2 \cdot \alpha) .
$$

As shown in [MOP07], $\rho_{\max }$ can be expressed as

$$
\rho_{\max }=\rho\left(H_{S, k_{c}}, \check{P}_{S, \hat{t}}\right) * \hat{p} * \sqrt{\frac{\operatorname{Var}\left(\check{P}_{S, \hat{t}}\right)}{\operatorname{Var}\left(P_{S, \hat{t}}\right)}},
$$


where, $P_{S, \hat{t}}$ and $\check{P}_{S, \hat{t}}$ are all power consumption points and the points related to leakage points at time $\hat{t}$ in the samples, respectively. Coefficient $\rho\left(H_{S, k_{c}}, \check{P}_{S, \hat{t}}\right)$ solely depends on the accuracy of the attacker's simulation or power model about the targeted device, and is set to the most conservative value 1 . The variances $\operatorname{Var}\left(P_{S, \hat{t}}\right)$ and $\operatorname{Var}\left(\check{P}_{S, \hat{t}}\right)$ are from the device characteristics, and are assumed to be equal to make our analysis independent of devices. Thereby, we have

$$
\rho_{\max }=\hat{p} .
$$

Now if we combine Equation 3.5 and Equation 3.8, and set $\alpha=0.1$ (that gives $z_{1-\alpha}=1.282$ ), then we can calculate the lower bound of the number of samples $\mathcal{N}$ to observe a noticeable $\rho_{\max }$ as follows,

$$
\mathcal{N}=3+\frac{13.148}{\ln ^{2}\left(\frac{1+\hat{p}}{1-\hat{p}}\right)} .
$$

Now, we define the robustness $\mathcal{R}$ of the secret key $K$ against DPA attacks as the total amount of time needed to gather $\mathcal{N}$ samples:

$$
\mathcal{R}=\mathcal{N} * \mathcal{P},
$$

where $\mathcal{P}$ is the occurrence period of AES having the secret key $K$.

\subsubsection{Intrusion Detection}

Besides communication confidentiality and side-channel resistance, detecting potential intrusions to the system is another important security aspect in the context of embedded systems. Intrusions [LJ97] are the malicious activities that try to access secret information or violate predefined security policies of a target system. An intrusion detection (ID) application actively monitors network and/or system activities and status changes, e.g. critical variables and log records, for possible intrusion evidences. We consider a host-based intrusion detection technique [YD03] that runs as an independent task in the system. To ensure detection effectiveness, it is recommended to employ both signature-based and anomaly-based intrusion detection techniques [SM07]. The signature-based technique tries to recognize 
known threats from patterns of existing attacks, while the anomalybased technique tries to find possible malicious activities with suspicious behaviors that deviate from normal system executions.

There exist various techniques for building ID models, either via manual knowledge engineering or automatic model abstractions, and detecting intrusions. For example, the automatic approaches presented in [HFS98, LSM99] are prominent techniques for developing ID applications in embedded systems. Both can be used for signaturebased and anomaly-based IDs. In this thesis, we assume a hybrid ID task, in which both techniques cooperate for achieving the best detection accuracy, co-existing with application tasks. Considering the limited memory size on embedded platforms, we assume that the models of normal behaviors stored in memory are obtained using the approach presented in [HFS98], which finds unique system call sequences of length 10, and exhibits low memory footprint. The technique in [LSM99] is only used for finding the models of known malicious attacks to be stored in memory. At run-time, the ID task checks the system execution traces for potential intrusions by comparing with both the pre-stored rules of normal behaviors and signatures of attacks.

The ID application task can be adjusted according to the desired trade-off between detection accuracy and consumed processor bandwidth. The only assumption is that higher detection accuracy will consume more processor bandwidth. In principle, we do not restrict to any particular forms in which the accuracy/overhead trade-off is captured. For the sake of simplicity, we shall assume that the ID task has a given nominal execution time $\mathcal{E}_{I D}$ (with respect to the highest supply voltage of the processor), and, at each release, it examines all necessary information for possible intrusion evidences. Meanwhile, the ID task may be activated with different periods $\mathcal{P}_{I D}$, depending on the available resources and also on the estimated level of threats. The longer the interval between two successive ID task releases, the easier the intrusion evidence can be hidden or even erased. Thus, the accuracy and promptness of the intrusion detection is directly influenced by the release period $\mathcal{P}_{I D}$. We define the intrusion detection 
accuracy $(I D A)$ as follows,

$$
I D A=\frac{\mathcal{P}_{I D}^{\min }}{\mathcal{P}_{I D}},
$$

where $\mathcal{P}_{I D}^{\min }$ is the shortest period that the ID task needs to be released in order to achieve the best detection performance. Note that our proposed techniques are not tied to any particular ID technique and accuracy quantification. Other variants of ID implementations and quantifications can be adapted as long as an accuracy vs. resource overhead trade-off relation can be provided. 


\section{Chapter 4}

\section{Design of Secure Distributed Embedded Systems}

$W^{\text {E shall present our design techniques for achieving confidential }}$ tems. We present two approaches to efficiently implement protection schemes depending on the architecture of the computational units in the system. In the first approach, we focus on achieving the highest affordable confidentiality protection on the architecture in which computational nodes are resource-limited microprocessors. In the second approach, we consider nodes that also contain reconfigurable FPGA areas that can be used for accelerations. The real-time properties are guaranteed in both approaches. The problem to be solved, which builds upon static scheduling of a set of dependent tasks on multiple processing resources, is known to be NP-hard [Ull75]. To solve the design problems, we first produce constraint logic programming (CLP) formulations, and solving a CLP formulation exactly will return the optimal solution for the corresponding problem. However, this is computationally expensive and, hence, efficient heuristics are proposed as alternatives. Note that the presented techniques can be applied both in the early system design phase and on the off-the-shelf systems. 
The rest of this chapter is organized as follows. Section 4.1 introduces the common application model shared in the two design problems. Section 4.2 and 4.3 detail the respective hardware architectures, and formulate the two problems, as well as present the proposed techniques and experimental evaluations. The chapter is summarized in Section 4.4.

\subsection{System Model}

\subsubsection{Architecture Model}

The hardware architecture is a distributed hardware platform as discussed in Section 3.1.1, and composed of two parts, a set of computational nodes and a communication bus which handles message communications. Each computational node has an embedded processor. All the tasks are executed in software on the processors. This is the architecture we consider for our first design problem (Section 4.2). In the second case (Section 4.3), we assume that a computational node consists of both a processor and an FPGA accelerator, which can be used to offload certain computations to enhance system performance. The processors in both cases can be of different types and processing capabilities.

\subsubsection{Application Model}

In this chapter, we capture an application as a directed acyclic task graph $G(\mathcal{T}, E)$ that is mapped to a distributed hardware platform as described in the previous section. $\mathcal{T}$ represents a set of nonpreemptive tasks that need to be executed in the system. The mapping from tasks to computational nodes is given by a function $\mathcal{F}$ : $\mathcal{T} \rightarrow P$, where $P=\left\{p_{1}, p_{2}, \ldots, p_{n}\right\}$ is the set of available computational nodes. $\mathcal{F}(\tau)$ is the node that task $\tau$ is mapped to for execution. The bus is considered as a special resource that only handles the message transmissions. The execution time (ET) of a task $\tau$ is given as a constant value $c$.

The edges $E$ of graph $G$ capture the data dependencies between tasks. An edge $e \in E$ from $\tau_{s}$ to $\tau_{t}$ indicates that there exists a data 


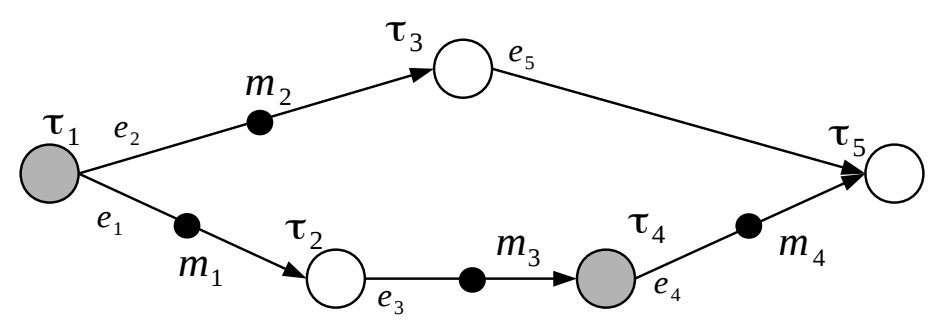

Figure 4.1: A simple application with 5 tasks

dependency between $\tau_{t}$ and $\tau_{s}\left(\tau_{t}\right.$ depends on $\left.\tau_{s}\right)$. If $\tau_{s}$ and $\tau_{t}$ are mapped to the same processing node, i.e., $\mathcal{F}\left(\tau_{s}\right)=\mathcal{F}\left(\tau_{t}\right)$, then the data transfer is achieved by memory copies, and no message will be transmitted over the bus. Otherwise, a message $m$ will be transferred from $\mathcal{F}\left(\tau_{s}\right)$ to $\mathcal{F}\left(\tau_{t}\right)$ over the bus, after $\tau_{s}$ finishes. The message transmission times of messages are also given.

The task mapping is defined by the designer, so the set of messages is known. Figure 4.1 depicts an illustrative application containing 5 tasks and 5 edges. Tasks $\left\{\tau_{1}, \tau_{4}\right\}$ and $\left\{\tau_{2}, \tau_{3}, \tau_{5}\right\}$ are mapped to processor $p_{1}$ and $p_{2}$, respectively. As the consequence of this mapping, there is no message transmission on edge $e_{5}$ in Figure 4.1, as $\mathcal{F}\left(\tau_{3}\right)=$ $\mathcal{F}\left(\tau_{5}\right)$. On the other hand, the edge $e_{3}$, for example, will lead to a message communication $m_{3}$ sent from $\mathcal{F}\left(\tau_{2}\right)$ to $\mathcal{F}\left(\tau_{4}\right)$. A task can only start execution after all of its preceding tasks have finished and its input data have been successfully received. The whole application must complete its execution before an end-to-end delay $\mathcal{D}$, known as its global deadline.

\subsection{Confidentiality Optimization}

Now, let us first look at the case of hardware nodes without FPGA acceleration. In order to achieve good overall security protection in the context of tight resource limits and stringent timing constraints, we need to find the best system-affordable cryptographic protections to the internal communication, during the early stages of system design and optimization. 


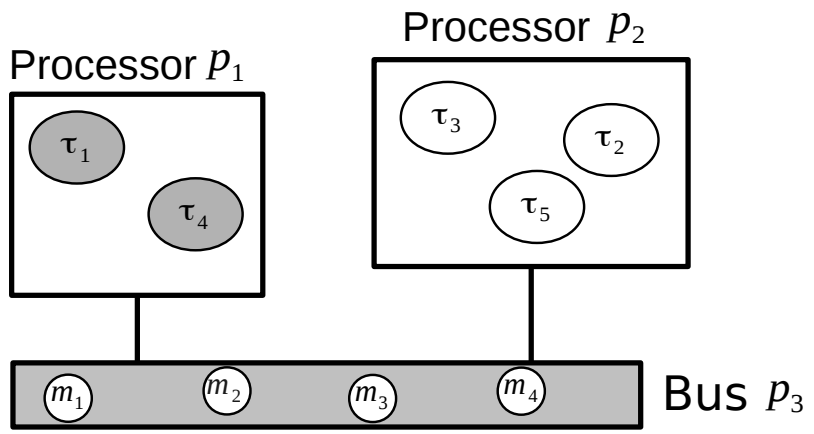

Figure 4.2: A processor-based platform

\subsubsection{Motivational Example}

Let us consider the case that the application shown in Figure 4.1 is mapped to the hardware architecture depicted in Figure 4.2. The task to processor mappings follow the example mentioned in Section 4.1.2, and are captured in the figure. The communication messages $\left\{m_{1}, m_{2}, m_{3}, m_{4}\right\}$ are transferred over the bus, denoted as $p_{3}$. The ETs of the tasks are set as $c_{1}=110, c_{2}=90, c_{3}=170, c_{4}=110$, and $c_{5}=150$, respectively, and the payload transmission times of all the messages are set as 15 . The global deadline is 700 time units.

In order to protect the communication against malicious snooping, encryption and decryption should be deployed on the messages. If no $\mathrm{E} / \mathrm{D}$ task is performed, the shortest end-to-end delay of this application is 535 time units. The corresponding schedule is shown in Figure 4.3. As can be observed, the system currently has time slacks that potentially can be utilized to perform encryption and decryption operations within the global deadline of 700 time units.

Let us assume that the designer wants to apply RC6 [RRSY98] on the messages, of which the pre/post-whitening and one E/D round both take 2 time units to finish on both processors. A simple approach to protect the communication of the application would be to maximize the protections on all the messages. Assuming the designer wants to use RC6 variant of 20 rounds, then he will get a schedule as shown in Figure 4.4. However, the end-to-end delay of the application is now 


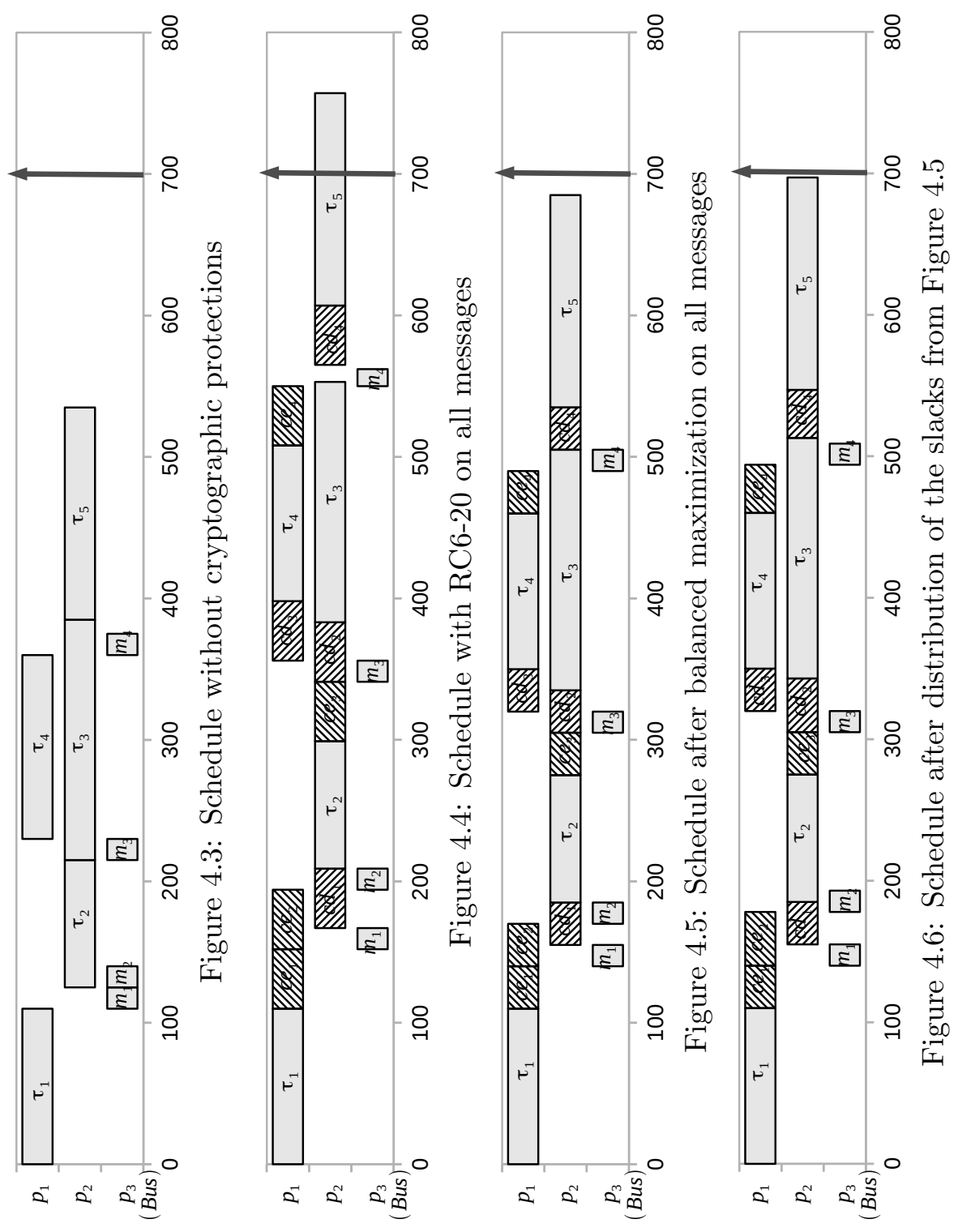


Table 4.1: Results of different solutions in Figure 4.3-4.6

\begin{tabular}{|l|c|c|c|c|c|}
\hline Solution & $m_{1}$ & $m_{2}$ & $m_{3}$ & $m_{4}$ & Schedule \\
\hline No E/D & 0 & 0 & 0 & 0 & Figure 4.3 \\
\hline $\begin{array}{l}\text { Fixed high-protection } \\
\text { for all messages }\end{array}$ & 20 & 20 & 20 & 20 & $\begin{array}{c}\text { Figure 4.4 } \\
\text { (Not schedulable) }\end{array}$ \\
\hline Even slack distribution & 14 & 14 & 14 & 14 & Figure 4.5 \\
\hline Further rounds increment & 14 & 18 & 14 & 16 & Figure 4.6 \\
\hline
\end{tabular}

757 , which violates the deadline constraint of 700 time units. So this solution is not feasible.

Another alternative solution would be to distribute the number of E/D rounds evenly to all messages. The best possible solution without violating the global deadline in this case is to assign $14 \mathrm{E} / \mathrm{D}$ rounds to each message transmitted over the bus, and the corresponding schedule is illustrated in Figure 4.5. Assigning 16 or more rounds to all messages at the same time would violate the imposed deadline of 700. Nevertheless, the schedule in Figure 4.5 still contains small time slacks, which means that the number of $\mathrm{E} / \mathrm{D}$ rounds for certain individual messages can still be increased.

Figure 4.6 illustrates a solution in which the available slacks have been further utilized to increase the security level of selected messages. Now the applied numbers of E/D rounds for the messages become 14, 18, 14 and 16, in which two messages are further encrypted by 4 and 2 more rounds, respectively. This is the optimal solution we can achieve. The number of rounds used by the messages in the aforementioned solutions are presented in Table 4.1.

\subsubsection{Problem Formulation}

Now we shall formulate the design optimization problem. As already mentioned, the goal is to find the best affordable confidentiality protections for the internal communication. That is, we want to optimize the protection for messages transmitted over the bus using IBCs, under the available processing power and the imposed timing 
constraints. The optimization problem is decomposed into two subproblems that are performed in two steps.

\subsubsection{Step I}

In the first step, we want to find the maximal number of rounds $N$ that all E/D tasks can perform. In other words, we aim at finding a schedulable solution of the system within the deadline, in which $N$ rounds of the chosen IBC are used by all E/Ds. This step maximizes the smallest number of rounds globally (see Figure 4.5 and the fourth row in Table 4.1).

\subsubsection{Step II}

In the second step, the system may still have extra time slack due to unbalanced workload and the diverse processing power of different processors. Hence, we can further increase the security strength of the internal communication by utilizing the available processor bandwidth. Thereby, the E/D modules running on the smallest number of rounds and generating the plaintext-cipher pairs (refer to Section 3.2.1.2) are reduced. As a result, the potential vulnerability of the system is further dwindled. This is particularly effective if different E/D pairs use distinct secret keys to encrypt their messages.

In this step, we want to increase the number of $E / D$ rounds as much as possible until the designer specified threshold, e.g., RC6-20, is reached, while also trying to provide a certain balance between the individual security degrees of the messages. These two aspects are captured by the following cost function that drives the optimization:

$$
\operatorname{Cost}=\alpha * \operatorname{Avrg}(X)-\beta * \operatorname{StandDev}(X)
$$

in which

$$
\begin{gathered}
\operatorname{Avrg}(X)=\frac{1}{n} * \sum_{i=1}^{n} x_{i} \\
\operatorname{StandDev}(X)=\sqrt{\frac{1}{n} * \sum_{i=1}^{n}\left(x_{i}-\operatorname{Avrg}(X)\right)^{2}}
\end{gathered}
$$




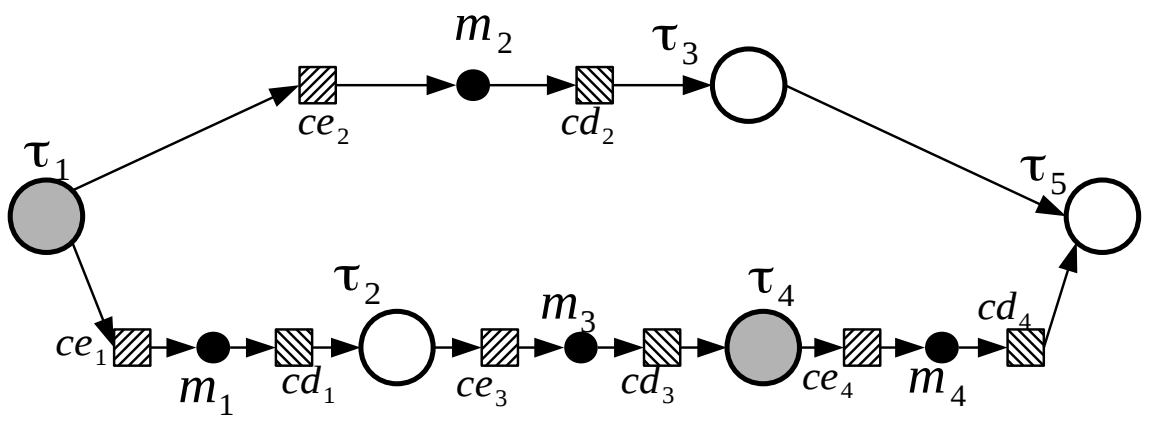

Figure 4.7: Reconstructed task graph of Figure 4.1

$\alpha$ and $\beta$ are the designer-provided weights depending on how balanced the security levels of different messages are desired.

For the sake of illustration purposes, we consider that all messages on the network share the same security requirement. However, the proposed approach can be generalized in a straightforward way if messages have different security requirements. For example, in Step II, the numbers of E/D rounds of different messages can be weighted with different coefficients to capture their actual security demands.

\subsubsection{Proposed Techniques}

Let us consider again the application in Figure 4.1 with mapping in 4.2. In order to keep the messages confidential, we have to perform message E/Ds. Each message will be encrypted before being sent over the bus by the source processor, and decrypted when received by the destination processor. To capture these operations in our representation, we shall make the procedure of the two cryptographic operations explicit. The newly added tasks for encryption and decryption are mapped to the same processors as the corresponding sending and receiving tasks. For example, encryption task $c e_{1}$ is mapped to processor $p_{1}$, while the corresponding decryption task $c d_{1}$ to processor $p_{2}$. The reconstructed application and updated task mappings are illustrated in Figure 4.7 and Figure 4.8, respectively. 


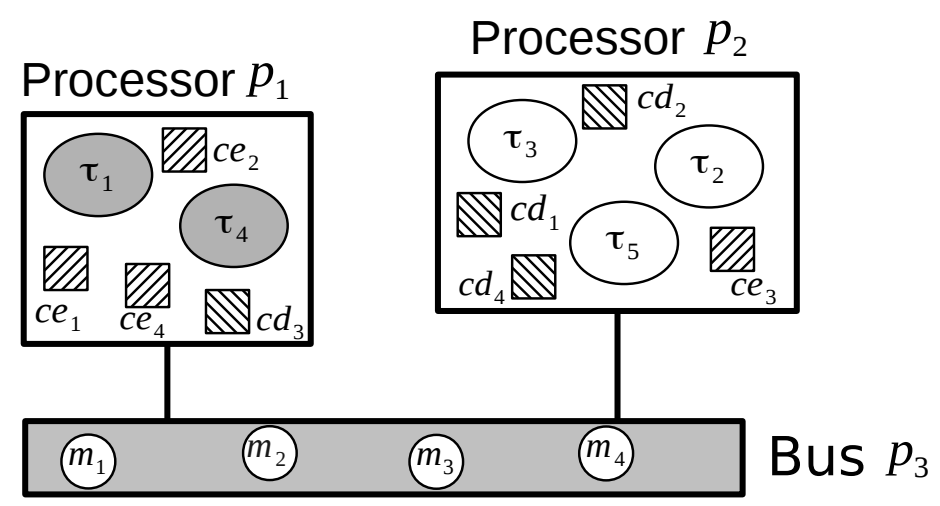

Figure 4.8: New task mapping from Figure 4.2

\subsubsection{CLP Formulation}

We first formulate the optimization problem using constraint logic programming (CLP) in which the problem is represented as a process of constraint satisfaction using a set of clauses capturing the constraints. Then the program is passed to a CLP solver that tries to find the optimal solution using various acceleration methods, such as branch and bound search. However, the complexity of finding the optimal solution by CLP still grows exponentially as the problem size grows. The reason of formulating the problem in CLP is to compare the later presented techniques with the optimums returned by the CLP solver.

Now let us present the CLP constraints grouped in two sets. The first set captures the dependencies of the original task graph, e.g., the encryption task $c e_{1}$ needs to be executed after task $\tau_{1}$ in Figure 4.7. The second set represents the schedulability constraints, e.g., the very last task $\tau_{5}$ in Figure 4.7 must successfully finish its execution before 700 time units. The CLP constraints we used are as follows.

Dependency constraints This set of constraints reflects the structure of the task graph. Each task is allowed to execute only after all 
its parent tasks have finished, i.e.,

$$
\begin{aligned}
& \forall \tau_{i} \in \mathcal{T} \text { and } \forall \tau_{j} \in \operatorname{Parent}\left(\tau_{i}\right), \\
& \qquad \operatorname{StartTime}\left(\tau_{i}\right) \geq \operatorname{StartTime}\left(\tau_{j}\right)+\operatorname{ET}\left(\tau_{j}\right)
\end{aligned}
$$

Schedulability constraints There are three types of constraints in this set, which together guarantee that the obtained result is schedulable within the global time constraint, and, in addition, the final schedule is correct.

- Execution time constraints:

The non-E/D tasks have constant ETs, while the E/D tasks have variable ETs that are related to the optimization variable $x_{i}$ as stated in Equation 3.4. So, in order to capture this behavior, we assign fixed values as the ETs for non-E/D tasks, and restrict the ETs of E/D tasks to a domain containing all the possible values that they can take with respect to all the designated number of rounds.

- Parallelization and sequence constraints:

The target application is mapped to a distributed platform. Therefore, the tasks on different processors can run in parallel, while the executions of different tasks on the same processor must not overlap with each other.

- Deadline constraint:

As all dependencies have been defined, we formulate the deadline constraint as follows: the last task of the application must finish its execution before the deadline $D$, i.e.,

$$
\operatorname{StartTime}\left(\tau_{\text {last }}\right)+E T\left(\tau_{\text {last }}\right) \leq D .
$$

If multiple tasks can be the last one in execution, meaning that none of them has a succeeding task, then a "virtual" last task with 0 execution time is added into the task graph that depends on all the potential last tasks. Then the deadline constraint is checked on this virtual task instead. 
Optimization objectives The two steps of the optimization have different objectives. In Step I, the objective is to find the maximal number of rounds $N$ that all the E/D pairs can perform without violating any constraint, including the final deadline constraint. While in Step II, the objective is to maximize the cost presented in Equation (4.1) based on the number $N$ obtained from Step I.

\subsubsection{Heuristic Approach}

Although the CLP formulation outlined above returns the optimal solutions, it cannot scale to large systems due to its computational complexity. In this section, we describe our heuristic alternative for solving the optimization problem which can handle large designs efficiently. The heuristic approach is based on two well-known algorithms, list scheduling (LS) [EPPD00] and simulated annealing (SA) [KJV83]. The former handles the schedulability test, and the latter optimizes the system with respect to the cost function.

Step I is achieved by gradually adding two rounds to all E/D tasks, whose ETs are also increased by the ETs of doing two rounds of RC6 on corresponding processors, until the system cannot be scheduled within the deadline limit. LS must be applied on the system for schedulability check after each increment of the rounds. This is because that, after each increment, ET of the E/D tasks changes. Consequently, the critical path determining the new end-to-end delay of the system may be different from the one before. Therefore, we cannot simply derive the end-to-end delay by adding the extra ETs to the length of the original critical path. The final obtained value of $N$ is the largest number of rounds that all the E/D tasks can perform without breaking the system schedulability, and will be used as the initial value for Step II. The pseudocode for this step is shown in Algorithm 1.

After the first step, there may exist time slacks that some E/D task pairs can use to undertake extra encryption and decryption rounds. So distributing these slacks in a smart way is crucial for getting closer result of Cost (Equation 4.1) to the optimum. We have used a SAbased heuristic driven by the second objective to approach good so- 


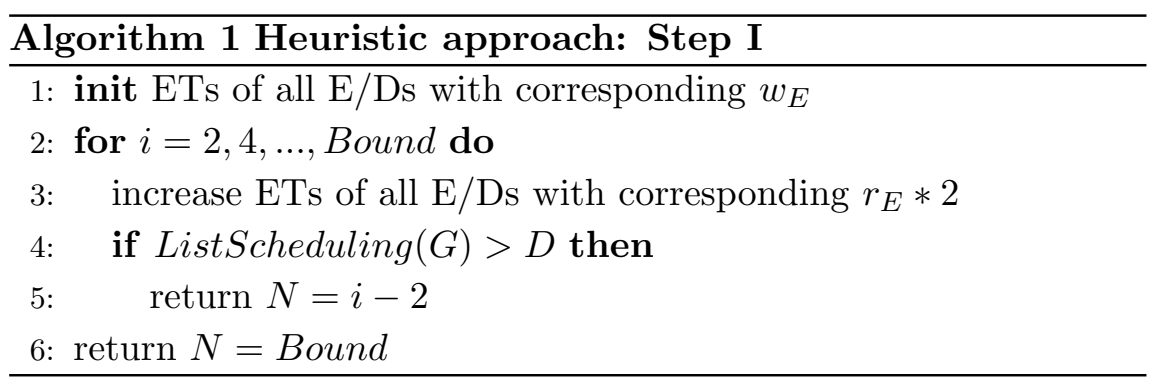

lutions. At the beginning, Cost and all $x_{i}\left(i \in\left\{1,2, \ldots, n_{M}\right\}\right)$ are initialized with currentcost and $N$, and the ETs of the E/Ds are also initialized correspondingly. Then SA randomly selects one E/D pair, e.g., $c e_{i}$ and $c d_{i}$, to manipulate each time. Both increment and decrement manipulations are allowed and randomly decided to be performed on $x_{i}$, but decrement moves leading to $x_{i}<N$ are prohibited. If the updated system can be scheduled by the list scheduling algorithm, then the program proceeds to check whether currentcost is higher than Cost. Otherwise, this pair of $c e_{i}$ and $c d_{i}$ are restored to the previous state. Now if currentcost is higher than Cost, then Cost is updated as currentcost. If not, a random number $r n \in(0,1)$ is generated and compared with $\exp (-\delta / t p)$, where $t p$ is the current temperature (control parameter for the SA algorithm), and $\delta$ is the cost decrement caused by this move. The current state and currentcost are kept only in case $r n<\exp (-\delta / t p)$. This helps the search procedure to escape from a local optimum.

The above optimization procedure will terminate when no improvement is found in a certain number of consecutive steps. The set of decision variables $x_{i}\left(i \in\left\{1,2, \ldots, n_{M}\right\}\right)$ hold the numbers of rounds for all $\mathrm{E} / \mathrm{D}$ pairs. And the system schedule is retrievable from the LS schedule table. The pseudocode for the second step is given in Algorithm 2. The SA parameters, including initial temperature, temperature reduction scheme and stopping condition, are set accordingly on different problem sizes from our observations in extensive experiments. 


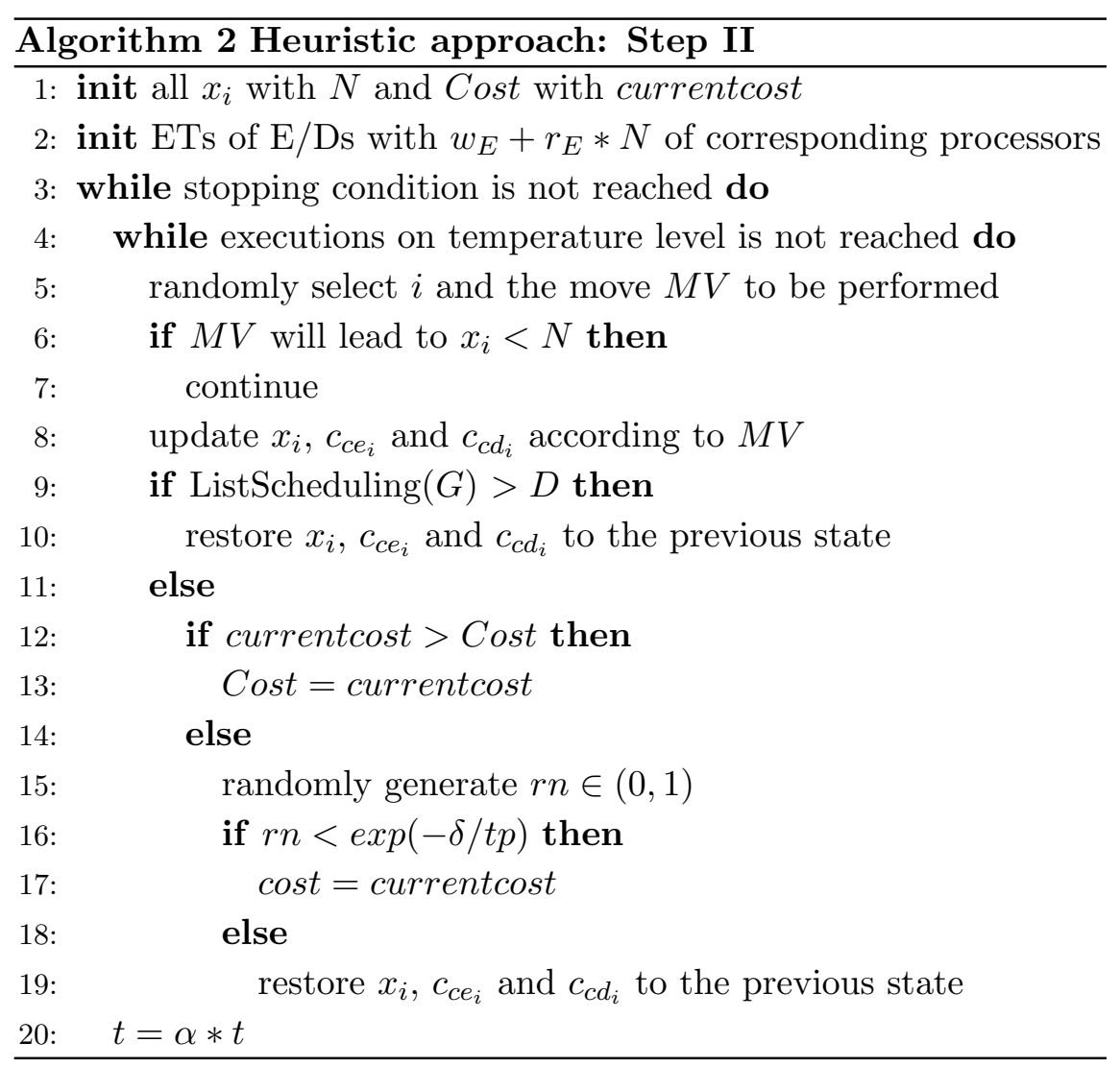

\subsubsection{Experimental Results}

We have performed experiments on randomly generated task graphs with 10, 15, 20, 30, 40, 50, 60, 70, 80, 90 application tasks (excluding the later added $\mathrm{E} / \mathrm{D}$ tasks) that were mapped on 2, 3, 3, 4, 4, 5, $5,6,6,7$ processors respectively. On each problem size, 50 different applications have been randomly produced. All experiments were performed on a Linux machine having a four-core Intel Xeon CPU with $2.66 \mathrm{GHz}$ frequency and $8 \mathrm{~GB}$ RAM.

The experiments with the CLP formulations were implemented in ECLiPSe constraint programming system [AW06], and were conducted with a timeout setup. If the ECLiPSe engine can find the optimal solution of an experiment before the timeout restriction, it returns the optimum for this application. Otherwise, it stops execu- 
tion without producing any result. This setup is used to restrict the overall optimization time of the extensive CLP experiments; otherwise, the CLP engine may run for extremely long time for some cases, and even may not terminate. The computational complexity of the CLP executions is therefore characterized by two parameters. One is the average execution time of those experiments that terminate and return optimal solutions. The other is the percentage of cases that fail to retrieve the optimums within the timeout restriction. In contrast, the proposed heuristic-based techniques will always terminate, and produce good solutions. Note that CLP formulation is problem specific, that is, each test application and task to processor mapping gives a different set of constraints. It is impossible to manually formulate CLP problems for all the test applications. So we developed an automatic CLP code generator that takes a task graph and given mapping, and returns a complete CLP formulation.

We set the timeout bounds of Step I and Step II as 300 and 1800 seconds, respectively, in all CLP experiments. The comparison of the average execution time (of both steps) of the finished CLP instances and corresponding heuristic executions is illustrated in Figure 4.9. The CLP experiments successfully found the optimal solutions for all the 50 test applications only on the smallest size with 10 tasks in the application. For graph size 15 and 20, there were 35 and 22 cases, respectively, out of 50 in which the optimums were retrieved before the timeout. While, starting from size 30, the CLP engine cannot anymore find the optimal solution for any of the CLP formulations, so their execution times are dismissed in Figure 4.9.

As we can see, the execution time of the CLP experiments grows exponentially as the graph size increases. Therefore, finding the optimal solution using the CLP formulation, is only feasible for very small applications. Figure 4.9 shows that the execution time of the proposed heuristics is much smaller than that of the CLP solutions. Of course, the execution time grows also aggressively due to the nature of the simulated annealing algorithm, but the average execution time of the heuristic is only around 900 seconds even for the biggest task graphs (with 90 non-E/D tasks).

The result comparison of the two approaches is presented in Fig- 


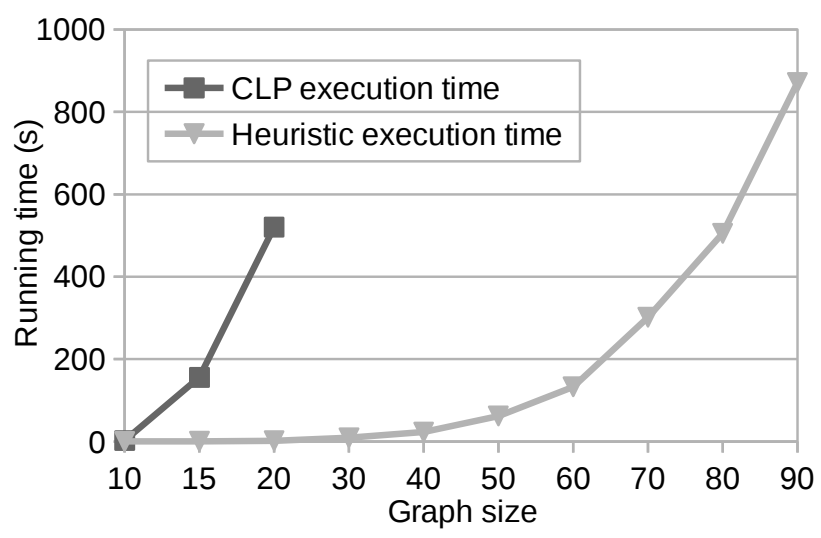

Figure 4.9: Average execution time of finished CLP experiments and heuristic approach

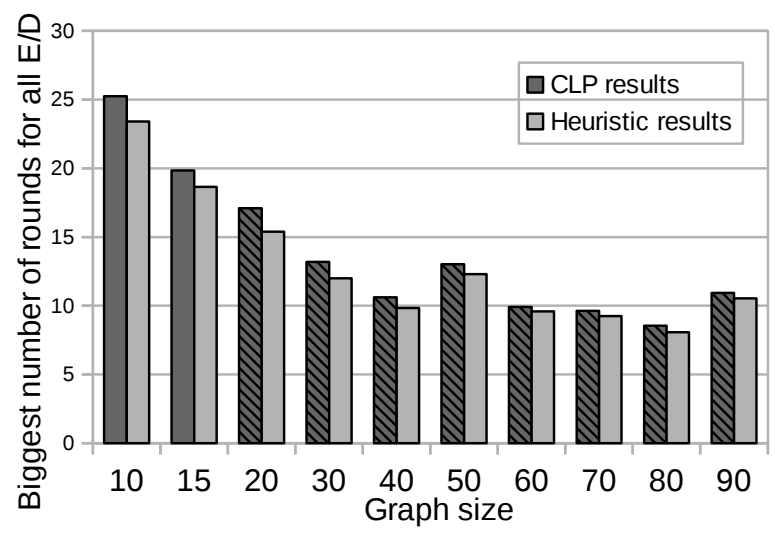

Figure 4.10: Result comparison of step I

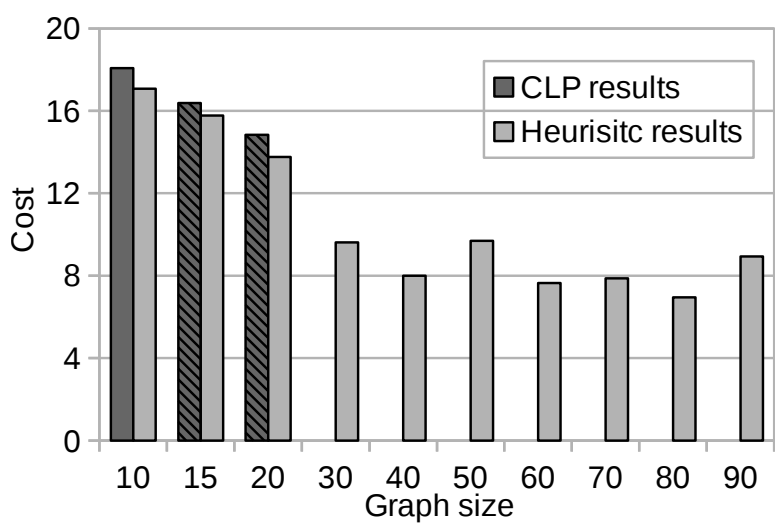

Figure 4.11: Result comparison of step II 
ure 4.10 and 4.11 for Step I and II, respectively. When comparing the results, the experiments that CLP failed to find the optimal solutions before timeout are eliminated. So we only analyze the CLP-obtained optimums and their correspondences returned by the proposed heuristics. For example, only 22 experiments of graph size 20 are used to generate the data in Figure 4.11. In both Figure 4.10 and 4.11, the dark gray bars without hatchings (left ones for size 10 and 15 in Figure 4.10, and for size 10 in Figure 4.11) indicate that all the CLP experiments found the optimal solutions, while the rest with hatchings present the results of only the finished CLP executions. The light gray bars are the results obtained by our heuristic approach. Figure 4.10 illustrates the comparison of $N$ for Step I. Averagely, the results produced by the heuristic is $93.83 \%$ of the optimums. In Step II, the costs were obtained by setting the weights as $\alpha=0.7$ and $\beta=0.3$. As shown in Figure 4.11, the final Cost returned by our heuristic approach and the optimal solutions retrieved by CLP are presented, and $94.5 \%$ of the optimal result can be achieved by the presented heuristic. From graph size 30, the results of CLP formulations are missing since none of the experiments found the optimal solution within our timeout bound.

\subsubsection{A Real-Life Case Study}

We also evaluated the proposed techniques on a real-life application, i.e., an adaptive cruise controller (ACC), similar to the one described in [Pop03]. The ACC application automatically maintains a safe following distance from the preceding vehicle. It also has the possibility of autonomous changes of the maximum speed depending on the speed-limit regulations, and assists the driver to brake in case of emergency.

The ACC application is composed of 22 tasks and 15 message communications. The application is constrained by a global deadline of 500 time units. The tasks are distributed to three microprocessors connected by a bus. The original task graph and the ETs of the tasks are shown in Figure 4.12, and the task-to-processor allocation is illustrated in Figure 4.13. 


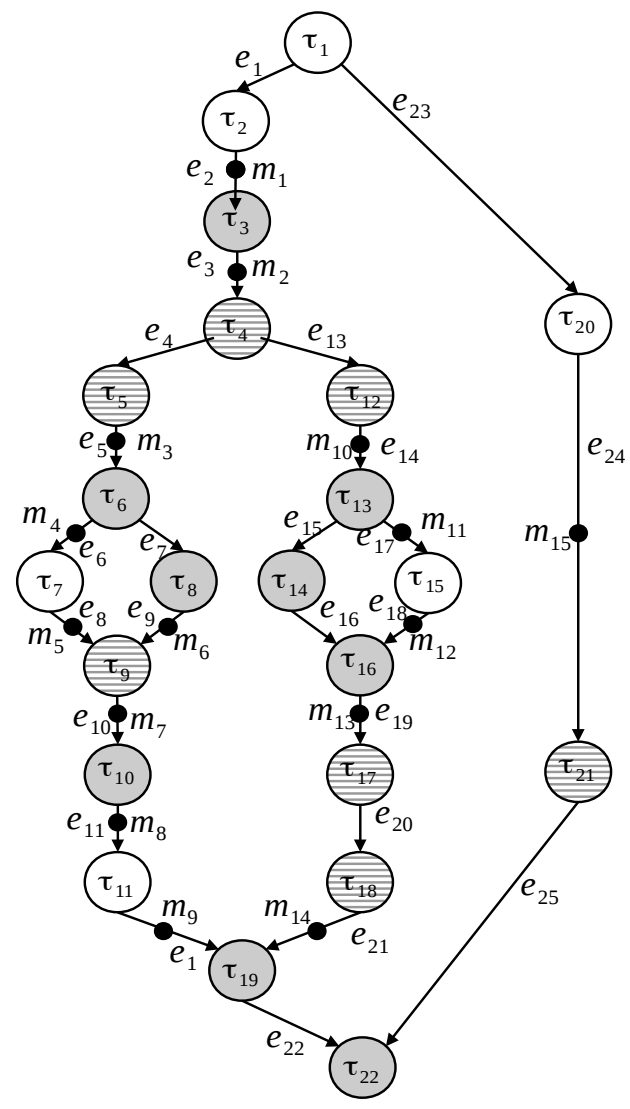

Process mapping

Processor $p_{1}: \tau_{1}, \tau_{2}, \tau_{7}$, $\tau_{11}, \tau_{15}, \tau_{20}$

Processor $p_{2}: \tau_{3}, \tau_{6}, \tau_{8}$, $\tau_{10}, \tau_{13}, \tau_{14}, \tau_{16}$, $\tau_{19}, \tau_{22}$

Processor $p_{3}: \tau_{4}, \tau_{5}, \tau_{9}$, $\tau_{12}, \tau_{17}, \tau_{18}, \tau_{21}$

Bus $p_{4}: m_{1}, m_{2}, m_{3}, m_{4}$, $m_{5}, m_{6}, m_{7}, m_{8}, m_{9}, m_{10}$, $m_{11}, m_{12}, m_{13}, m_{14}, m_{15}$

Payload comm. time

$c_{m_{1}}=1, c_{m_{2}}=1, c_{m_{3}}=1, c_{m_{4}}=1$,

$c_{m_{5}}=1, c_{m_{6}}=1, c_{m_{7}}=1, c_{m_{8}}=1$,

$c_{m_{9}}=1, c_{m_{10}}=1, c_{m_{11}}=1, c_{m_{12}}=1$,

$c_{m_{13}}=1, c_{m_{14}}=1, c_{m_{15}}=1$

Execution time

$c_{\tau_{1}}=0, c_{\tau_{2}}=27, c_{\tau_{3}}=22$,

$c_{\tau_{4}}=18, c_{\tau_{5}}=19, c_{\tau_{6}}=13$,

$c_{\tau_{7}}=33, c_{\tau_{3}}=12, c_{\tau_{0}}=25$,

$c_{\tau_{10}}=16, c_{\tau_{11}}=18, c_{\tau_{12}}=21$,

$c_{\tau_{13}}=5, c_{\tau_{14}}=10, c_{\tau_{15}}=23$,

$c_{\tau_{16}}=15, c_{\tau_{17}}=6, c_{\tau_{18}}=14$,

$c_{\tau_{19}}=15, c_{\tau_{20}}=21, c_{\tau_{21}}=15$,

$c_{\tau_{22}}=0$

Figure 4.12: An adaptive cruise controller

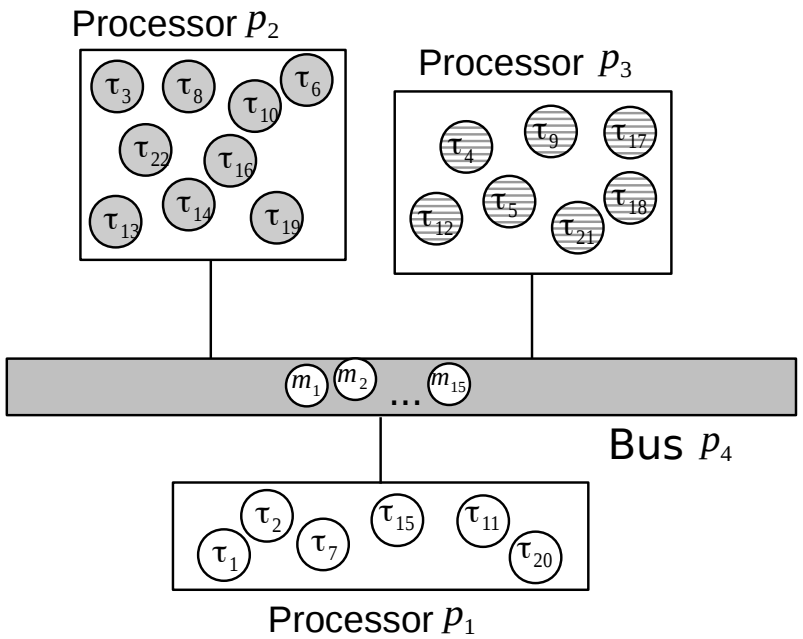

Figure 4.13: Hardware architecture of ACC 
Table 4.2: Result comparison of the ACC application

\begin{tabular}{|l|c|c|c|c|}
\hline & $N$ & Cost & Time 1 & Time II \\
\hline CLP & 12 & - & 9 & - \\
\hline Heuristic & 12 & 10.59 & 0.03 & 2.315 \\
\hline
\end{tabular}

The results returned by CLP and our heuristics are presented in Table 4.2, in which $N$ is the maximal number of rounds that all E/D tasks can handle, and Cost is the final optimization result obtained with $\alpha=0.7$ and $\beta=0.3$. Time I and Time II indicate the execution time (in seconds) for solving the first and second step, respectively. The CLP formulation for the Step II cannot find the optimal solution using any of the available search acceleration methods within our timeout setup of 1800 seconds. So no result is given in the "Cost" and "Time II" columns of CLP formulation. As can be observed, our heuristic found identical $N$ as the optimum in the first step, and returns the result within a very short period.

\subsection{Implementation Optimization}

Now let us look at another type of hardware architecture of the end computational node, in which each node is formed of a microprocessor and some attached FPGA area. The microprocessors in the system can be of different types and architecture as in the previous section. The FPGA co-processor shares the same memory with the processor, and is used to offload certain task executions. Since tasks mapped to FPGA can run in parallel with the tasks on processor of the same node (if no data dependency), and many tasks can be executed more efficiently in hardware than software, the system performance can be highly accelerated.

The characteristics of FPGAs match well with the core operations of IBCs, i.e. bit-substitution, XOR, and lookup table operations. In fact, the IBCs usually have good suitability, high performance, and small area consumption on FPGAs [EYCP01, DPR00]. In addition, 
VHSIC Hardware Description Language (VHDL) and Verilog implementations of IBC algorithms are also easily accessible ${ }^{1}$. Therefore, we only consider offloading the $\mathrm{E} / \mathrm{D}$ tasks in the following discussions to simplify the designer's role in using the presented techniques. By offloading and parallelizing the E/D tasks, the global performance will then be enhanced. Moreover, due to the fact that the life cycle of cryptographic algorithms is significantly lower than the life time of most RTESs (which may be used for tens of years), we can flash the FPGAs with up-to-date algorithms when needed without replacing the hardware, which is much more convenient comparing with having application-specific integrated circuit (ASIC) hardware implementations of given cipher algorithms.

Additionally, as already mentioned in Section 3.1.1, modern FPGA devices also allow modification of only a part of the gate array (called partial dynamic reconfiguration capability, abbr. as PDR). This capability offers even more flexibilities to the system designers, since such PDR enabled FPGAs can have part of the device reconfigured at run-time, while the rest keeps operational. Meanwhile, the FPGAs without PDR capability can only be reconfigured off-line. We will consider both kinds of FPGAs in this section. Such platforms with FPGA co-processors usually are designated to be more powerful in order to accommodate more challenging distributed applications. For such systems, we want to find the minimal total FPGA area that can help the system to implement the desired IBC protections on all message exchanges as well as to meet the deadline constraint.

\subsubsection{Motivational Example}

Let us consider the application $G(\mathcal{T}, E)$ of Figure 4.14 with task to computational node mapping given as Figure 4.15. The on-board FPGA co-processors beside the microprocessors are depicted by the hatched areas. The application consists of tasks $\mathcal{T}=\left\{\tau_{1}, \tau_{2}, \tau_{3}, \tau_{4}, \tau_{5}\right\}$ and data dependencies $E=\left\{e_{1}, e_{2}, e_{3}, e_{4}, e_{5}\right\}$. There are four message transmissions, i.e., $\left\{m_{1}, m_{2}, m_{3}, m_{4}\right\}$, based on the given map-

\footnotetext{
${ }^{1}$ Otherwise, the designer has to develop FPGA modules for all the application tasks.
} 


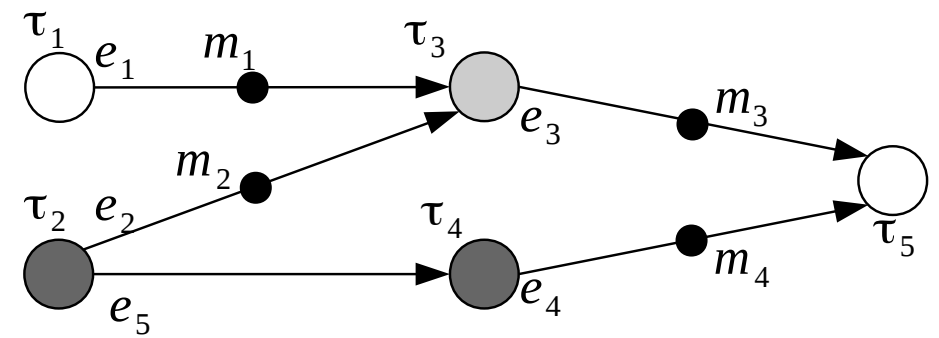

Figure 4.14: Another illustrative application

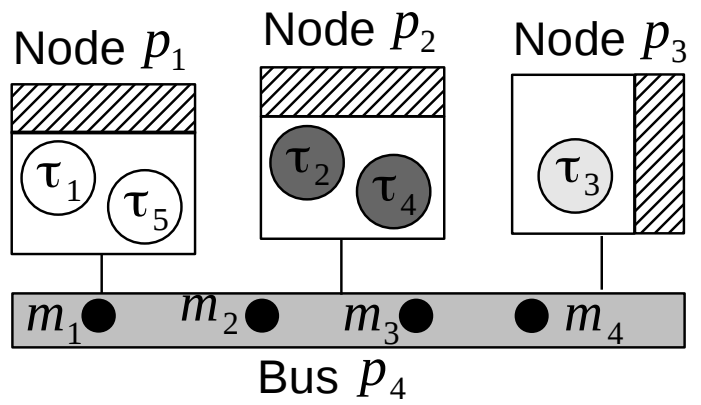

Figure 4.15: An FPGA-accelerated architecture

ping. Task $\left\{\tau_{1}, \tau_{5}\right\},\left\{\tau_{2}, \tau_{4}\right\}$ and $\left\{\tau_{3}\right\}$ are mapped to the computational nodes $p_{1}, p_{2}$ and $p_{3}$, respectively. The communication messages $\left\{m_{1}, m_{2}, m_{3}, m_{4}\right\}$ are transferred over the bus (denoted as $p_{4}$ ). The ETs of the tasks are $\{60,180,50,140,150\}$, respectively, and the transmission times (TTs) of all the messages are assumed to be 20 time units. The system is constrained by a global deadline $D$ of 600 time units.

As mentioned in the previous section, we need to encrypt the messages before sending, and decrypt them after receiving on another node to make the internal communication confidential using software only implementations of RC6. In this section, we consider that the $\mathrm{E} / \mathrm{D}$ tasks can be implemented either in hardware or software. However, implementing all cryptographic operations in software very likely cannot provide a schedulable solution due to the imposed security and 
real-time constraints. But we can make use of the onboard FPGAs to accelerate the $\mathrm{E} / \mathrm{D}$ processes. Let us consider the scenario that the designer choose to implement the AES standard of the 128-bits version on all messages. Since the AES decryption performs similar operations as the encryption, but in reverse order, (and replaces several inner operations, e.g., SHIFTROWS, with their inverse operations), the required amount of FPGA area and the execution times of AES E/D tasks are roughly the same. Therefore, we assume that the area overhead of implementing one E/D task in FPGA is 1 unit. Consequently, the extra hardware overhead to system is the total FPGA units used.

Let us illustrate the schedules corresponding to different solutions. Assuming that all the messages are in plaintext manner (i.e., no E/D operations were performed), the corresponding schedule is shown in Figure 4.16. The end to end delay of the application is 490 units, and, as can be observed, we have some time slacks that can be used to perform E/D operations on the messages. The ETs of E/D tasks are determined by the function in Equation 3.4. In this system, the designated AES variant to be implemented is AES-128bit version with rounds $x_{i}=10$ on all messages. The values of $\left(w_{E D_{i}}, r_{E D_{i}}\right)$ of software and FPGA implemented E/D tasks are set to be $(4,10)$ and $(2$, $4)$, respectively.

If we do not introduce FPGA units into the system (for new designs) or utilize the existing FPGAs (for off-the-shelf systems), we have to carry out all $\mathrm{E} / \mathrm{D}$ tasks in software on the microprocessors, as discussed in the previous section. In this case, in order to satisfy the deadline of 600 , we can only encrypt the messages with 3 rounds, which is not a satisfactory solution. The schedule can be found in Figure 4.17. A simple approach that tries to reach the required number of 10 rounds, while respecting all constraints, is to accommodate all E/D tasks in FPGA. This leads to an end-to-end delay of 594 and hardware overhead of 8 as shown in Figure 4.18.

If we look closer at the schedule in Figure 4.18, we can find that the encryption operations of $m_{2}$ and $m_{4}$ may share the same FPGA unit without violating the deadline constraint. The same goes for decryption operations of $m_{1}$ and $m_{2}$. In addition, we can save one 


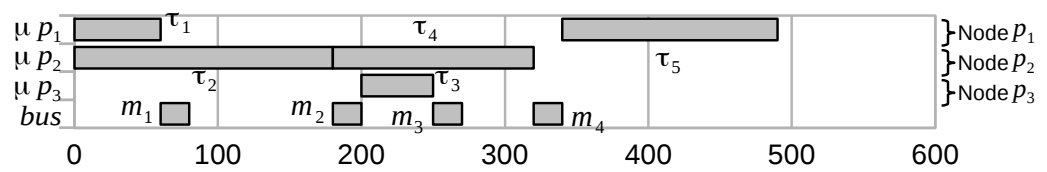

Figure 4.16: Schedule without cryptographic protections

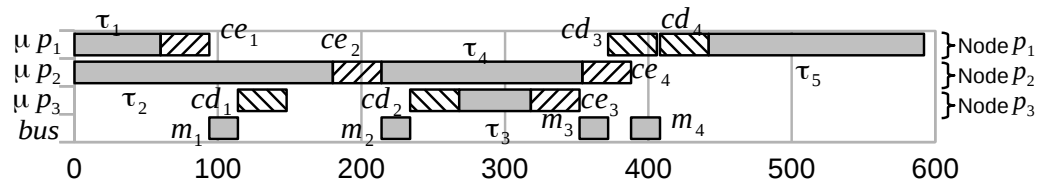

Figure 4.17: Schedule of software only solution

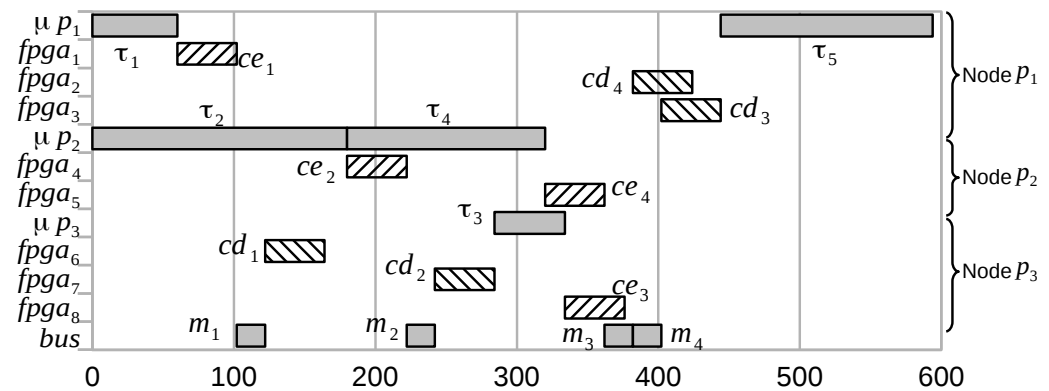

Figure 4.18: Schedule of assigning FPGA to all E/Ds

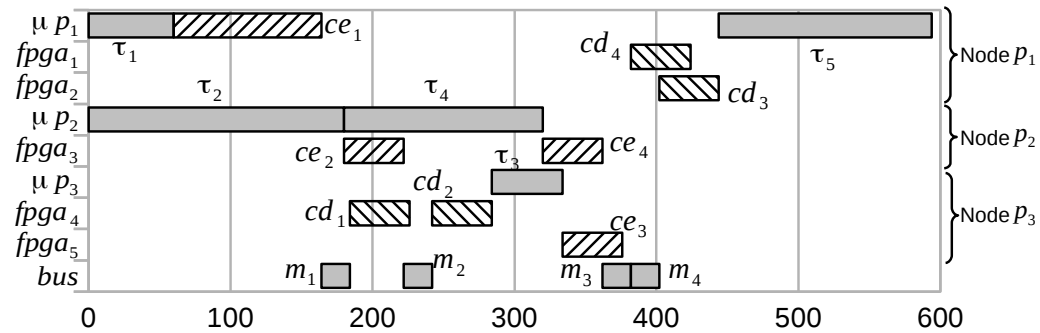

Figure 4.19: Schedule of the optimal solution for static FPGA

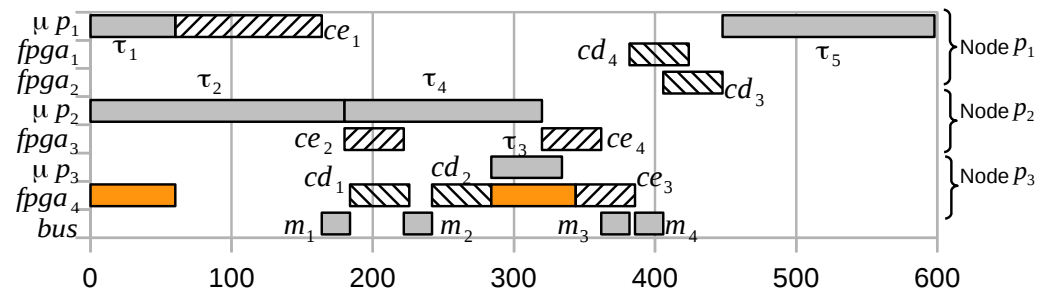

Figure 4.20: Schedule of the optimal solution for PDR-enable FPGA 
Table 4.3: Results of different solutions in Figure 4.16-4.20

\begin{tabular}{|l|c|c|c|}
\hline Solution & Rounds & HW overhead & Schedule \\
\hline No E/D & 0 & 0 & Figure4.16 \\
\hline Software & 3 & 0 & Figure4.17 \\
\hline Straight-forward & 10 & 8 & Figure4.18 \\
\hline Static configuration & 10 & 5 & Figure4.19 \\
\hline PDR enabled & 10 & 4 & Figure4.20 \\
\hline
\end{tabular}

extra FPGA unit by implementing the encryption operation of $m_{1}$ in software. By this, we save three FPGA units without sacrificing the protection level, as can be observed in Figure 4.19. By this, the system is able to encrypt all messages with the required 10 rounds of AES, assisted by 5 FPGA units. In fact, this is the optimal solution, if FPGAs with static configuration are used.

Furthermore, if the FPGA supports $\mathrm{PDR}^{2}$, we can also achieve the same level of security using even less hardware area. If the reconfiguration time is assumed to be 60 units, the schedule of the optimal solution is illustrated in Figure 4.20. The FPGAs on $p_{1}$ and $p_{2}$ only undertake E/D tasks of the same kind (all are encryptions, or all are decryptions), so they do not need to be reconfigured at run-time. While, the FPGA unit on $p_{3}$ must be reconfigured dynamically (depicted as orange rectangles), since it is shared by both kinds of cryptographic operations. The numbers of achieved rounds and hardware overheads of all the above solutions are listed in Table 4.3.

\subsubsection{Problem Formulation}

The application is modeled as a task graph $G(\mathcal{T}, E)$ that is mapped to a distributed hardware platform. The ETs and TTs of ordinary tasks and messages are known. The values of software and FPGA implemented $w_{E D_{i}}$ and $r_{E D_{i}}$ are given. The hardware cost implied by an E/D task is considered to be 1 unit as already mentioned. The application is constrained by a given end-to-end delay $D$, and

\footnotetext{
${ }^{2}$ Remind that PDR stands for partial dynamic reconfiguration
} 
is required to meet a specific confidentiality protection level, that is given by the set $X=\left\{x_{1}, x_{2}, \ldots, x_{n}\right\}$. The variable $x_{i}$ indicates an IBC variant with $x_{i}$ rounds that need to be applied on message $m_{i}$. Different messages can have different security demands reflecting their importance or specific design requirement.

We are interested in finding the minimal overall number of FPGA units $F N$ that need to be added into the system, such that the system reaches the designated confidentiality protection, and, at the same time, the real-time constraints are satisfied. Therefore, the objective is to find the appropriate implementation for all $\mathrm{E} / \mathrm{D}$ tasks and the corresponding system schedule that lead to the minimal hardware overhead. In the following sections, we shall present our proposed techniques and corresponding experimental evaluations on two different FPGA technologies, which are, FPGA with static configuration and with PDR capability.

\subsubsection{Proposed Techniques}

We first formulate the design optimization problem using CLP, and then present our heuristic approaches that can be employed even on large designs. In order to make the system more flexible to be scheduled and analyzed, we explicitly represent the message E/D operations as independent tasks using the same method as in Section 4.2.3. Remind that now each computational node has both a microprocessor and an FPGA co-processor to use, where the message E/D tasks can both be allocated to. Figure 4.21 represents the updated application corresponding to the one in Figure 4.14. For example, the encryption operation of message $m_{1}$ is explicitly captured as $c e_{1}$, and can be mapped to the microprocessor or an FPGA unit on computational node $p_{1}$.

\subsubsection{FPGAs with Static Configuration}

CLP formulation The CLP formulation for the statically configured FPGAs shares two sets of constraints with the ones presented in Section 4.2.3.1, which are the dependency constraints and the schedulability constraint. So we only focus on the new sets of constraints 


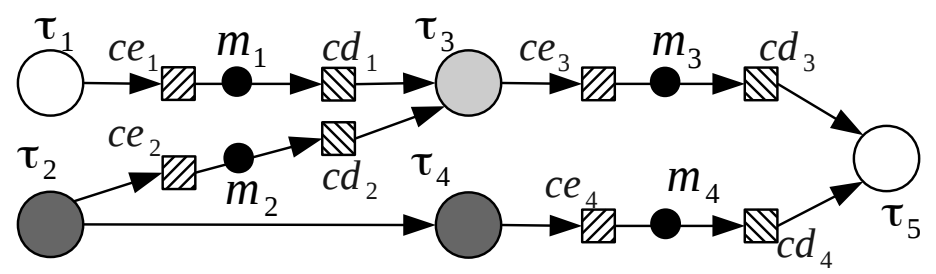

Figure 4.21: Reconstructed task graph of Figure 4.14

in this section. We denote an encryption or decryption task as $e d_{i}$ to simplify the following discussions.

- Implementation constraints

The first set of constraints restricts that an E/D task can only be implemented once, either in software or in FPGA, i.e.,

$$
s w_{e d_{i}}+\sum_{j=1}^{U} h w_{e d_{i}}^{j}=1 .
$$

where, $s w_{e d_{i}}, h w_{e d_{i}}^{j} \in\{0,1\} . e d_{i}$ is implemented in software if $s w_{e d_{i}}=1$, or on the $j$ th FPGA unit of $\mathcal{F}\left(e d_{i}\right)$ if $h w_{e d_{i}}^{j}=1$. $U$ is a constant that indicates the size of available areas on the FPGA co-processor. For existing off-the-shelf platforms, $U$ represents the actual available areas on a co-processor. For the design from scratch of a new system, $U$ is set to a large number only for CLP implementation purposes.

- Execution time constraints

Similar to the execution time constraints presented in Section 4.2.3.1, the non-E/D tasks and messages have fixed ETs and TTs. While, the ETs of E/D tasks depend on their actual implementations, i.e., in software or FPGA, as follows:

$$
E T\left(e d_{i}\right)= \begin{cases}w_{E D}^{\mu P}+r_{E D}^{\mu P} * x_{i}, & \text { if } s w_{e d_{i}}=1, \\ w_{E D}^{F P G A}+r_{E D}^{F P G A} * x_{i}, & \text { otherwise. }\end{cases}
$$


The values of $\left(w_{E D}^{\mu P}, r_{E D}^{\mu P}\right)$ and $\left(w_{E D}^{F P G A}, r_{E D}^{F P G A}\right)$ (refer to Equation 3.4) are the ET parameters of E/D tasks executed on processor and FPGA, respectively.

- Resource sharing constraints

The E/D tasks on the same computational node cannot share the same FPGA unit, if they are not of the same type. For example, if the $j$-th FPGA unit is occupied by the implementation of an encryption task with a certain IBC variant, then this unit may only be reused by the encryption tasks of the same IBC variant. This is formulated as

$$
\forall p_{i} \in P \text { and } \forall j \in\{1, \ldots, U\},
$$

However, this does not restrict that all the IBC variant of the same type should always share the same unit, since multiple FPGA implementations can be necessary when the deadline constraint is tight.

Optimization objective If a potential FPGA unit on $p_{i}$ is assigned with at least one E/D task, the corresponding FPGA areas have to be added into the system or occupied on the existing FPGA areas. Therefore, the optimization objective is to find the minimal number $F N$ of FPGA units that are needed, such that all constraints are satisfied. This can be represented as follows,

$$
F N=\sum_{\forall p_{i} \in P} \sum_{j=1}^{U} N_{j}^{\prime},
$$

where,

$$
N_{j}^{\prime}= \begin{cases}0, & \text { if } \sum_{\forall e d_{k} \text { on } p_{i}} h w_{e d_{k}}^{j}=0 \\ 1, & \text { otherwise. }\end{cases}
$$




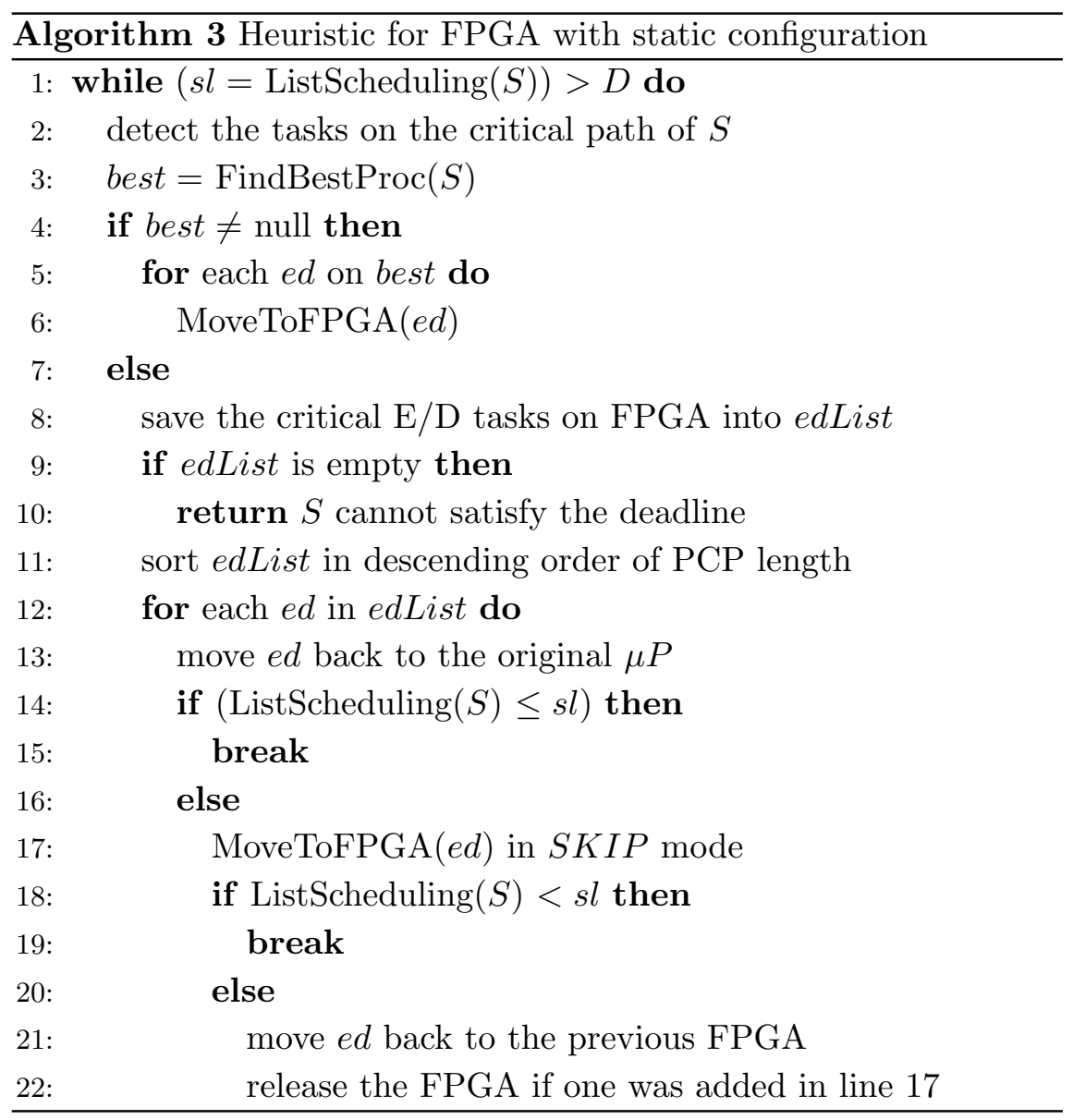

Heuristic Given the CLP formulation of a certain problem, a CLP solver will return the optimal solution that satisfies the constraints outlined above, if such a solution exists. Due to the complexity of solving CLP problems, we propose a heuristic alternative for handling large system designs that is divided into two parts, resource allocation and list scheduling [EPPD00]. The former controls the outer codesign problem of FPGA allocation and resource sharing. The latter tries to find a feasible system schedule. The pseudocode is shown in Algorithm 3.

We first detect the tasks on the critical path of the given system, $S$, i.e., a task graph with given mapping decisions, (line 2 of Algorithm 


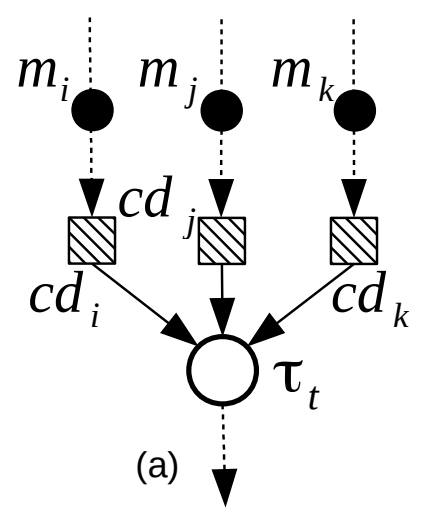

(b)

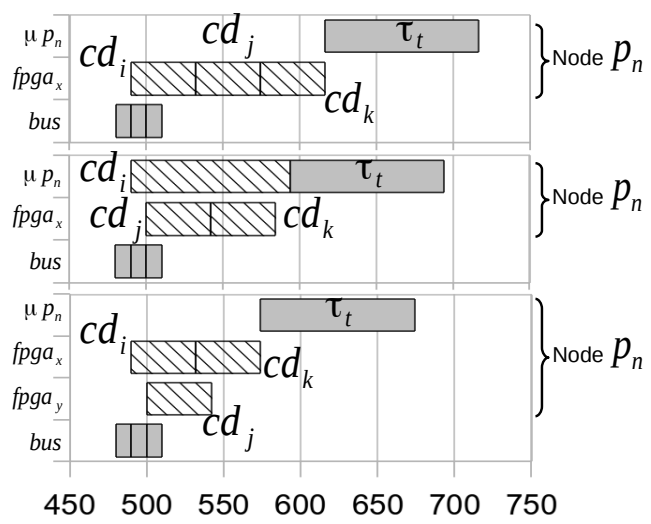

Figure 4.22: An example with 3 consecutive messages

3). Then FindBestProc $(S)$ returns the microprocessor carrying the most number of critical (on the critical path) software implemented encryption tasks (or decryption tasks) (line 3). If two microprocessors have the same number of critical encryption or decryption tasks, the one with the longest total partial critical path (PCP) length [EPPD00] of the critical tasks is chosen. After that, all encryption or decryption tasks on microprocessor best are moved to a new FPGA unit (line 5-6). As, in this case, an FPGA unit can only be configured for encryption or decryption tasks, the function MoveToFPGA $(e d)$ checks whether ed and each FPGA unit belong to the same computational node, and whether the unit is for the same type of IBC variant.

If there is no software implemented critical E/D task, the algorithm tries to find the critical E/Ds on FPGAs, and save them into a list edList (line 8). IF edList is empty, it means that no E/D task is left on the critical path. Hence, the application cannot be further accelerated by hardware implementation of E/D tasks, and, thus, the heuristic is terminated. Otherwise, we sort edList in descending order of their PCP lengths, and try to allocate each E/D task ed $\in$ edList in an appropriate implementation (line 11-22). Figure 4.22 is an illustrative example for this.

Assuming that the task graph in Figure 4.22 (a) is part of a bigger system. The ET of $\tau_{t}$ is 100 time units, and TT of the messages is 10 
time units. The $\left(w_{E D_{i}}, r_{E D_{i}}\right)$ values of software and FPGA implemented E/D tasks are assumed to be $(4,10)$ and $(2,4)$, respectively. The messages $m_{i}, m_{j}$ and $m_{k}$ are received at time 490, 500 and 510, respectively. Let us consider the following scenario. In order for the system to meet the global deadline, $\tau_{t}$ must finish its execution before 700 time units. Currently, all the three decryption tasks $c d_{i}, c d_{j}$ and $c d_{k}$ are on the PCP, and are allocated on the same FPGA unit leading to the schedule in Figure 4.22 (b). As can be noticed, $\tau_{t}$ finishes at time 716, which means that the global deadline will be violated. The algorithm tries to move $c d_{i}$ back to the $\mu P$ that it originally belongs to. This will lead to an end-to-end delay shorter than the previous case, so the algorithm keeps the current system setting, and breaks from the inner loop (line 13-15). The schedule can be found in Figure 4.22 (c).

In another case, if $\tau_{t}$ must finish before 680 time units, reallocating $c d_{i}$ back to the original microprocessor will not give a schedulable solution, so the algorithm tries to allocate the next task $c d_{j}$ in edList on an FPGA unit that it was not be mapped to before (line 17), e.g., a new FPGA unit possibly or another existing FPGA unit on the same computational node that is free for $c d_{j}$. This leads to the schedule in Figure 4.22 (d). Then, the algorithm breaks from the inner loop (line 19). Otherwise, it moves $c d_{j}$ back to the FPGA unit which it was mapped to previously, and release the FPGA unit if a new one was occupied or added into the system (line 21-22). The algorithm continues until the system can be scheduled within the global deadline, and returns the final solution. Or, it terminates when edList is empty, which means that the system cannot satisfy the deadline constraint while achieving the designated confidentiality protection.

We have two settings that help avoid deadlocks. Each E/D task has a history list keeping track of the FPGAs that it had been implemented on. If it needs to be moved to FPGA in SKIP mode (line 17), it avoids being moved to an FPGA unit in its history list. And we ignore the $\mathrm{E} / \mathrm{D}$ tasks that have been moved too many times, and keep them in the microprocessor or FPGA unit where it appears the most times. The length of the history list and value of maximal moves can 
be tuned for each problem size to reach the best optimization results.

\subsubsection{Partial Dynamic Reconfiguration}

PDR enabled FPGAs allow reconfiguring a portion of the FPGA areas, while the other parts remain functional. In this case, different types of operations, i.e. encryption and decryption tasks, can share the same FPGA unit, if there is enough time for reconfiguration in between. The PDR capability increases the flexibility of resource sharing, thereby reducing the hardware overhead.

CLP formulation The CLP formulation for the PDR enabled FPGAs is similar with the previous case, so we only focus on the ones that differ, namely, the resource sharing and reconfiguration constraints. The E/D tasks can be implemented on the same FPGA unit, which needs to be reconfigured if a task is of different kind with the previous one. We define a new kind of tasks, called the PDR tasks, to capture the reconfiguration procedures of the FPGAs. The ET of the PDR tasks can be 0 or $\mathcal{E}^{P D R}$. That is, a PDR task is a virtual task if its $\mathrm{ET}$ is 0, or a valid task otherwise with a constant execution time $\mathcal{E}^{P D R}$ given by the designer that reflects the partial dynamic reconfiguration overhead for an $\mathrm{E} / \mathrm{D}$ module.

- Partial dynamic reconfiguration:

The FPGA units must be reconfigured on the fly to support resource sharing between encryption and decryption operations. These constraints can be formulated as follows. 
$\forall p_{i} \in P$ and $\forall f p_{j} \in\left\{\right.$ all potential FPGA units on $\left.p_{i}\right\}$, $\forall e d_{s} \in\left\{\right.$ all E/D tasks on $\left.p_{i}\right\}$ $e d_{t}$ is the previous task of $e d_{s}$ and $h w_{e d_{t}}^{f p_{j}}=1$,

$$
E T\left(\tau_{e d_{s}}^{f p_{j}}\right)=\left\{\begin{array}{l}
0, \text { if }\left\{\begin{array}{l}
h w_{e d_{s}}^{f p_{j}}=0 \\
h w_{e d_{j}}^{f p_{j}}=1 \text { and } E\left(e d_{s}\right)=E\left(e d_{t}\right)
\end{array}\right. \\
\mathcal{E}^{P D R}, \text { if } h w_{e d_{s}}^{f p_{j}}=1 \text { and } E\left(e d_{s}\right) \neq E\left(e d_{t}\right)
\end{array}\right.
$$

where, $\tau_{e d_{s}}^{f p_{j}}$ is the PDR task for $e d_{s}$ on potential FPGA unit $f p_{j}$, and

$$
E\left(e d_{s}\right)=\left\{\begin{array}{l}
0, \text { if } e d_{s} \text { is an encryption task } \\
1, \text { if } e d_{s} \text { is a decryption task }
\end{array}\right.
$$

- PDR dependencies:

The executions of E/D tasks and PDR tasks on the same FPGA unit cannot overlap with each other. An E/D task can only start its execution after the module has been successfully reconfigured for its use, which is already captured by dependency constraints.

Optimization objective The objective is to minimize the total number of FPGA units that need be added into the system for new designs or to be occupied on existing systems, as mentioned in the previous static configuration approach (refer to Equation 4.7).

Heuristic The structure of our proposed heuristic for PDR enabled FPGAs is similar to that of Algorithm 3. We also guide the resource distribution by analyzing the $\mathrm{E} / \mathrm{D}$ tasks on the critical path. But when looking for the microprocessor best (line 3), the algorithm counts the number of critical E/D tasks of both encryption and decryption, since they can share the same FPGA unit via dynamic reconfigurations. If an E/D task ed needs to be moved to FPGA (the MoveToFPGA(ed) function), the algorithm only checks whether ed 
and each FPGA unit belong to the same computational node. In addition, we insert a set of new tasks capturing the reconfiguration operations when we schedule the application. In order to avoid deadlocks, the ideas of maximal moves and history lists are adopted from Section 4.3.3.1. To avoid repetitions, we shall skip the details of the algorithm.

\subsubsection{Experimental Results}

We have performed experiments on six sets of applications having 10, 15, 20, 40, 80 and 120 tasks (excluding E/D tasks) that are mapped to $2,3,4,7,10$ and 15 processors respectively. All experiments were performed on a Linux machine having a quad-core Intel Xeon CPU with $2.66 \mathrm{GHz}$ frequency and 8GB RAM. In the experiments, we set the $\left(w_{E D}, r_{E D}\right)$ values for software and FPGA as $(4,10)$ and $(2,4)$, respectively. The messages are assumed to have the same confidentiality requirement, that is AES-128 version with 10 rounds. We modified the automatic CLP code generator mentioned in Section 4.2.4 to obtain correct CLP formulations with the new properties specified in the previous sections. The formulated CLP problems were solved by the ECLiPSe constraint programming system [AW06], and were carried out with a timeout setup of 1800 seconds.

\subsubsection{FPGA with Static Configuration}

Due to the fact that solving the CLP problems does not scale even for middle-sized systems, we also compare our heuristic with a greedy straight-forward approach. This approach repeatedly assigns a new FPGA unit to take over the E/D tasks from the microprocessor that currently has the most number of $\mathrm{E} / \mathrm{D}$ tasks. It terminates when the system can be scheduled within the deadline, and returns the solution. Otherwise, it terminates when there is no software implemented E/D task left, which means that the system cannot be protected with the designated requirements, while satisfying the timing constraints, using this greedy approach.

For each set of applications, we have performed experiments on 20 individual applications that have the same number of application 


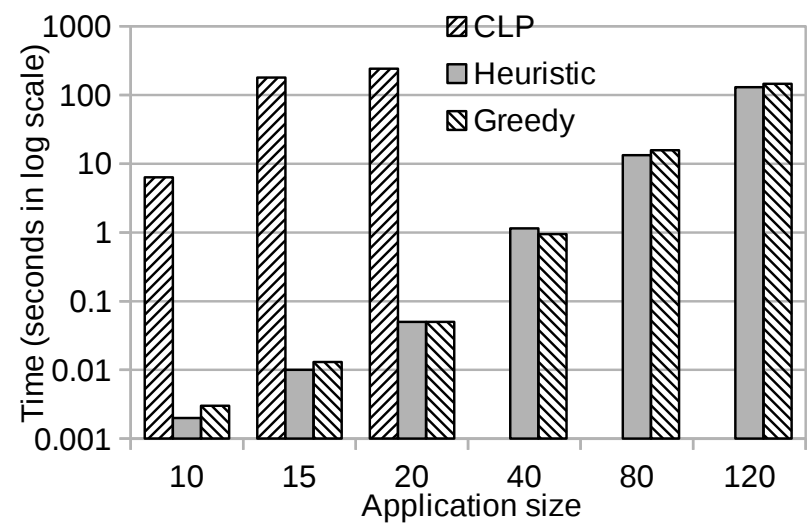

Figure 4.23: Optimization time with statically configured FPGA

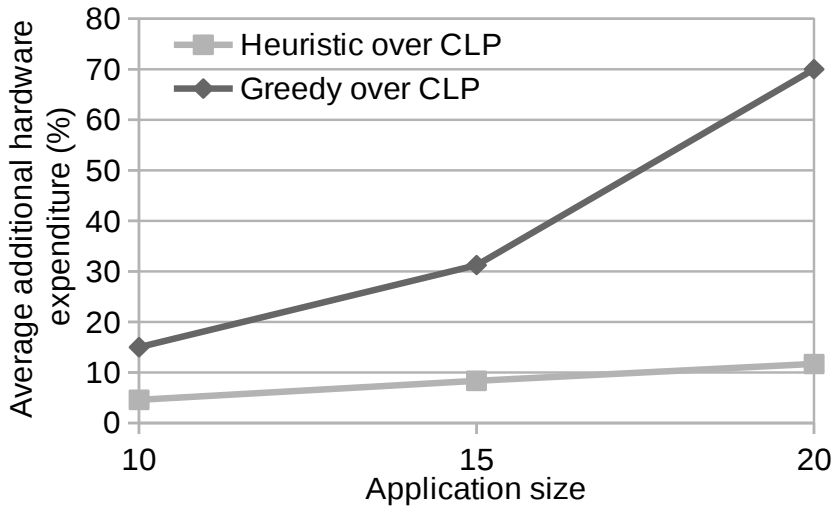

Figure 4.24: Results with statically configured FPGA (1)

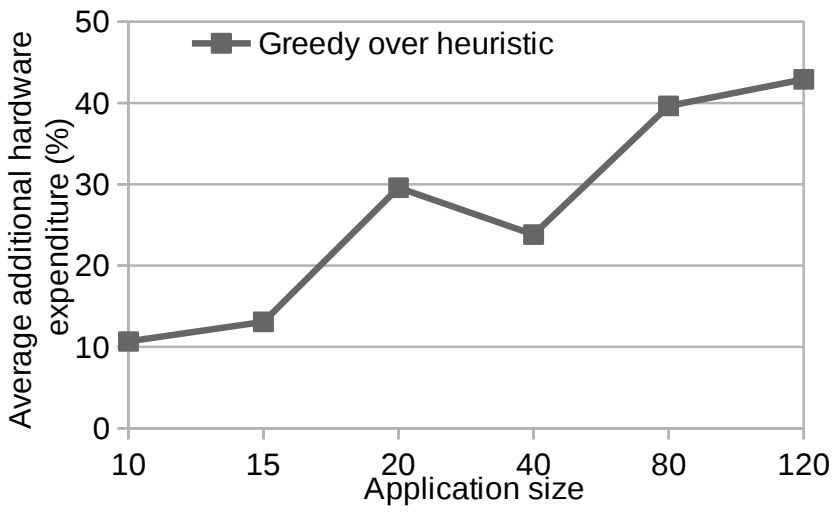

Figure 4.25: Results with statically configured FPGA (2) 
tasks and similar amount of bus communications. The average optimization times of CLP, our heuristic and the greedy approach are presented in Figure 4.23, in which the $y$-axis is in logarithmic scale. For the times of CLP, we only considered those experiments which terminated before the timeout (for example, in the case of application size 20, only 5 out of 20 CLP runs finished before the timeout). Starting from size 40, no CLP experiment could find the optimal solution within the timeout restriction.

When comparing the results obtained by two different approaches, we calculate the average additional hardware expenditure (AAHE) of one approach over the other. This is formulated as

$$
A A H E=\frac{1}{n}\left(\sum_{\forall i \in n} \frac{F N_{i}^{\prime}-F N_{i}}{F N_{i}}\right) * 100 \%
$$

where, $F N_{i}$ and $F N_{i}^{\prime}$ are the obtained results (refer to Equation 4.7) with the two approaches for the same application, and $n$ is the number of valid experiments, i.e., in which both approaches found solutions. Figure 4.24 presents the AAHE of our proposed heuristic over CLP and of the greedy approach over CLP for problem sizes of 10, 15, and 20 , in which at least some of the CLP formulations were solved. As can be observed, our heuristic approach provides very close results to the optimums. For example, on the largest application size, 20, where ECLiPSe managed to solve some experiments, our heuristic only requires about $11 \%$ extra FPGA units compared to the optimal solutions. Figure 4.25 depicts the AAHE of the greedy approach over our proposed heuristic on all application sizes. We can notice that our heuristic approach saves significant amount of FPGA units compared with the greedy method.

\subsubsection{Partial Dynamic Reconfiguration}

We have conducted the same set of experiments as those in static configuration considering FPGAs with PDR capability. The optimization comparison of the CLP and heuristic approach can be found in Figure 4.26. Starting from application size 40, no execution time is presented for the CLP formulation, since none of the experiments finished within the timeout limit. 


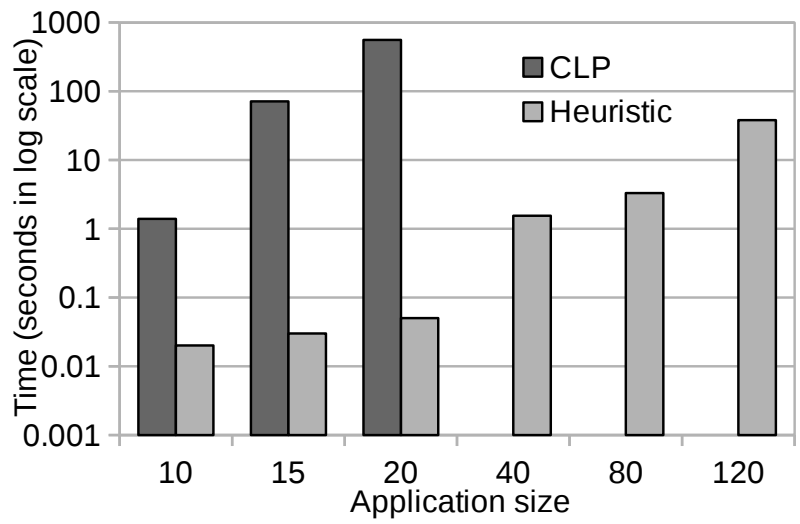

Figure 4.26: Optimization time with PDR enabled FPGA

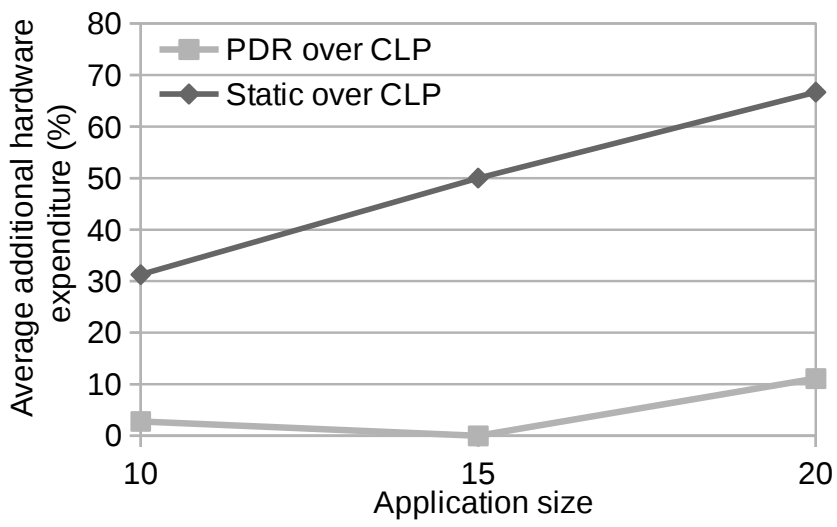

Figure 4.27: Results with PDR enabled FPGA (1)

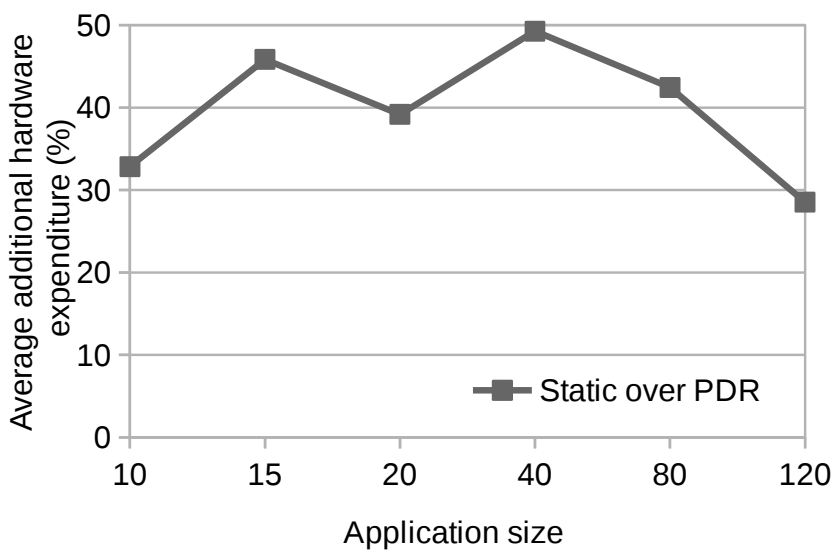

Figure 4.28: Results with PDR enabled FPGA (2) 
We use the same analytical method (Equation 4.9) as before to compare the quality of results. In Figure 4.27, we compare the optimal solutions obtained by CLP with the results returned by our heuristic approaches for both FPGA techniques. The experiments that CLP failed to find the optimum within the timeout setup are ignored. The hardware savings using PDR enabled FPGA over static configured FPGA for all application sizes are illustrated in Figure 4.28. It can be observed that our heuristic performs well comparing with the optimal results obtained from CLP, and achieves large FPGA unit savings (33\% on average over all problem sizes) if PDR-enabled FPGAs are used in the system.

\subsection{Summary}

In this chapter, we have presented our optimization techniques for protecting the confidentiality of internal communication using iterated block ciphers for distributed real-time embedded systems. We investigated two design problems with respect to two different architecture configurations, one with microprocessor only and the other with also FPGA co-processors. We first formulated the problems in CLP, and then proposed heuristic alternatives for solving the problems more efficiently both in terms of time and result quality.

The presented techniques can be either applied as a security patch on the off-the-shelf distributed RTESs, or employed in the early design phase of any new systems where the internal communication confidentiality is required. 


\section{Chapter 5}

\section{Design of Secure Multi-Mode Embedded Systems}

$T_{\text {embedded systems. While considering real-time and quality of }}^{\text {HIS chapter addresses the design challenges of secure multi-mode }}$ service requirements, we also study the emerging security and power efficiency demands. Modern RTESs very often are not developed for a fixed single purpose, but instead designed for undertaking various processing requests. This leads to the concept of multi-mode RTESs, in which the number and nature of active tasks change during runtime. Under dynamic situations, providing high performance along with various design concerns becomes a really difficult problem. We present our design optimization techniques that enhance quality of service under timing, security, and energy constraints. Due to the complexity of exactly solving this problem, we propose an efficient heuristic-based technique that generates near optimal solutions.

The rest of this chapter is organized as follows. Section 5.1 highlights the hardware and application model used in this chapter. Section 5.2 outlines the design objectives we face in each mode. Section 5.3 presents an illustrative example as a motivation. Section 5.4 formulates the overall design optimization problem. The proposed techniques are elaborated in Section 5.5. The experimental evalua- 


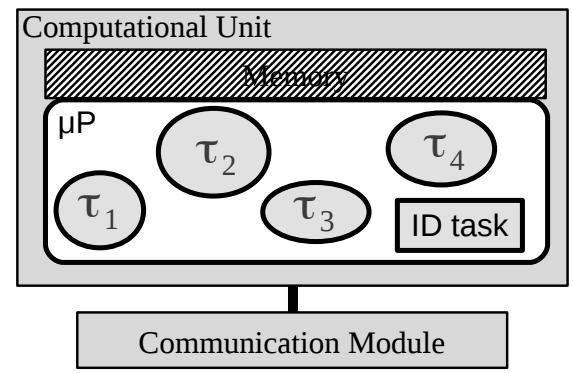

Figure 5.1: An illustrative multi-mode system

tions and a real-life case study are presented in Section 5.6 and 5.7, respectively. Section 5.8 summarizes the whole chapter.

\subsection{System Model}

\subsubsection{Hardware Model}

We consider a uniprocessor platform based on a DVFS-enabled embedded processor which handles all computations (Figure 5.1). As discussed in Section 3.1.2, the supply voltage (and implicitly the frequency) of the processor can be selected at run-time from a discrete set, depending on concrete operational requirements. If the real-time constraints are tight, the voltage (and frequency) could be increased in order to finish task executions earlier. If, on the contrary, the available energy in the system is limited, the voltage (and frequency) could be reduced in order to lower the power consumption.

The system has a communication module, via which it interacts, by wire or wirelessly, with other peers or service centers, and an onboard memory, that can be used to store pre-defined system configurations. In this chapter, a system configuration contains the resource allocation decisions, cipher variant selections for message E/Ds, period assignment for the intrusion detection (ID) task, and voltage/frequency decisions for all tasks for a certain mode. We shall detail these aspects in the following sections. 


\subsubsection{Application Model}

\subsubsection{Task Model}

At any moment in time on the system, there is a set of active tasks representing the current mode $M$ of the system. In this chapter, we consider the imprecise computation model for tasks [LSL $\left.{ }^{+} 94\right]$. That is, the tasks must deliver timely results and provide a minimal given quality of service. If longer execution time is available, better quality results can be produced. For example, an image processing task can deliver more accurate output if longer execution time is affordable. The tasks are preemptive and periodic. We assume that task executions are independent (i.e., without any precedence constraints or data dependencies).

Formally, a task $\tau_{i} \in \mathcal{T}$ is composed of a mandatory and an optional execution, and is associated with a set of design attributes denoted as $\left(\mathcal{E}_{i}^{m}, \mathcal{E}_{i}^{o}, \mathcal{P}_{i}, \mathcal{D}_{i}, \mathcal{L}_{i}, C_{\text {eff }}, \mathcal{Q}_{i}^{m}, \mathcal{F}_{i}\right) . \mathcal{E}_{i}^{m}$ is the execution time (ET) of the mandatory part. $\mathcal{E}_{i}^{o}$ is the maximal amount of time for the optional execution, i.e., the actual additional execution time $c_{i}^{o} \in\left[0, \mathcal{E}_{i}^{o}\right]$. Both $\mathcal{E}_{i}^{m}$ and $\mathcal{E}_{i}^{o}$ are nominal ETs, that is, they are obtained with respect to the highest available processor frequency $f^{\max }$. Thus, the actual ET of $\tau_{i}$ at frequency $f_{i}$ can be calculated as

$$
c_{i}\left(f_{i}\right)=\frac{\left(\mathcal{E}_{i}^{m}+c_{i}^{o}\right) \cdot f^{\max }}{f_{i}} .
$$

$\mathcal{P}_{i}$ is the release period of $\tau_{i}$, and $\mathcal{D}_{i}$ is its relative deadline $\left(\mathcal{D}_{i} \leq \mathcal{P}_{i}\right)$. The attribute $\mathcal{L}_{i}$ is the set of messages associated with task $\tau_{i}$. Each message $m_{i j} \in \mathcal{L}_{i}$ is associated with a length $l_{i j}$ (in number of blocks of the chosen IBC), i.e.,

$$
l_{i j}=\left\lceil\frac{m_{i j} \text { length in bits }}{\text { block length in bits }}\right\rceil,
$$

a weight $w_{i j}$ representing its relative importance, and a minimal quality of confidentiality (QoC) requirement, $Q o C_{i j}^{\min }$. $C_{e f f_{i}}$ is the effective switching capacitance of task $\tau_{i}$ reflecting its power hungriness (refer to Equation 3.1). $\mathcal{Q}_{i}^{m}$ is the constant quality of service (QoS) value produced by the mandatory execution of $\tau_{i}$. $\mathcal{F}_{i}$ is the 


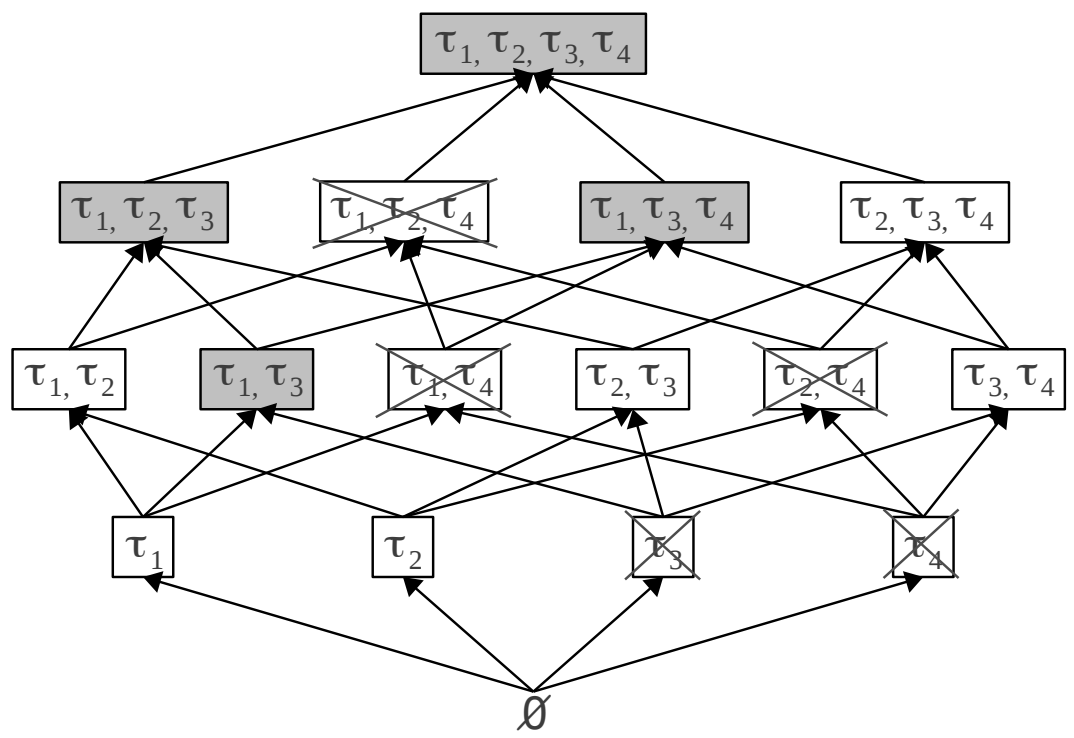

Figure 5.2: The Hasse diagram of modes for Figure 5.1

reward function that will be introduced in Section 5.2.1, expressing the amount of additional QoS produced by the extra execution $c_{i}^{o}$ of the optional part.

\subsubsection{Execution Modes}

All the tasks that might occur in the system are given as a set $\mathcal{T}=$ $\left\{\tau_{1}, \tau_{2}, \ldots, \tau_{n}\right\}$. At different points of time, the set of active tasks can be different and is dynamically changing at run-time. The currently active set of tasks defines the current mode $M \subseteq \mathcal{T}$. When new tasks come or existing tasks terminate, the system switches to a new mode. The complete set of modes for a given system is the power set of $\mathcal{T}$, denoted with $\mathcal{M}$, having cardinality $|\mathcal{M}|=2^{|\mathcal{T}|}$. However, certain modes can be excluded due to functionality constraints. In the rest of the chapter, we are interested only in the modes that can occur at run-time, and we shall refer to them as the functional modes $\mathcal{M}^{\text {func }} \subseteq \mathcal{M}$.

Mode $M \in \mathcal{M}$ is called a supermode of $M^{\prime} \in \mathcal{M}$, if $M^{\prime} \subset M$. Similarly, $M^{\prime}$ is called a submode of $M$. The sets of all supermodes 
and submodes of $M$ are denoted with $\overline{\mathcal{M}}(M)$ and $\underline{\mathcal{M}}(M)$, respectively. The mode containing all the tasks in $\mathcal{T}$ is referred to as the root mode. Functional modes that do not have any functional supermodes are called top functional modes, denoted by $\mathcal{M}_{\uparrow}^{\text {func }}$. Let us consider the example system in Figure 5.1. Four application tasks may occur in the system, i.e., $\tau_{1}, \tau_{2}, \tau_{3}$, and $\tau_{4}$. The corresponding partial order capturing the relations of all potential modes is depicted in Figure 5.2 as a Hasse diagram. The functionally excluded modes, e.g., $M^{124}=\left\{\tau_{1}, \tau_{2}, \tau_{4}\right\}, M^{14}=\left\{\tau_{1}, \tau_{4}\right\}, M^{24}=\left\{\tau_{2}, \tau_{4}\right\}$, and $M^{3}=\left\{\tau_{3}\right\}$, are marked out with a cross. The only top functional mode is $\mathcal{M}_{\uparrow}^{\text {func }}=\left\{\tau_{1}, \tau_{2}, \tau_{3}, \tau_{4}\right\}$, which is also the root mode.

\subsubsection{Scheduling Model}

We assume that the system is scheduled according to the earliest deadline first policy (EDF). Thus, whether the system is schedulable can be verified by a processor utilization test with task deadlines. First, the load caused by the execution of $\tau_{i}$, together with the potential $\mathrm{E} / \mathrm{D}$ tasks on its communication messages, with respect to its deadline, is

$$
U_{i}\left(f_{i}\right)=\frac{c_{i}\left(f_{i}\right)}{\mathcal{D}_{i}}+\sum_{m_{i j} \in \mathcal{L}_{i}} \frac{c_{i j}^{E D}\left(f_{i}\right)}{\mathcal{D}_{i}},
$$

where $c_{i j}^{E D}\left(f_{i}\right)$ is the E/D time (at the given processor frequency $f_{i}$ ) for message $m_{i j}$ using the chosen cipher variant $C_{i j}$, i.e.,

$$
c_{i j}^{E D}\left(f_{i}\right)=\frac{l_{i j} \cdot c_{C_{i j}} \cdot f^{\max }}{f_{i}} .
$$

The variable $c_{C_{i j}}$ is the corresponding ET (measured at the highest processor frequency $f^{\max }$ ) of the selected IBC variant $C_{i j}$ for encrypting/decrypting one block of message $m_{i j}$, and $l_{i j}$ is the length of message $m_{i j}$ in number of blocks.

Now we can test the system schedulability: a set of tasks is schedulable by EDF if and only if the total processor utilization with respect to deadlines is not more than $100 \%$. The utilization $U_{i}\left(f_{i}\right)$ of a task $\tau_{i}$ at a certain processor frequency $f_{i}$ was defined in Equation 5.2. 
Thereby, the schedulability of a certain configuration for mode $M$ can be examined by the following condition:

$$
U_{M}=\sum_{\tau_{i} \in M} U_{i}\left(f_{i}\right)+U_{I D} \leq 1 .
$$

The variable $U_{I D}$ is the utilization of the intrusion detection task, which we shall elaborate in Section 5.2.3.

\subsection{Design Objectives}

In this section, we shall introduce the design objectives of this chapter. Our ultimate design goal is that, no matter which mode and what constraints the system is running under, an efficient operation solution will be produced with respect to the four aspects described in the following subsections.

\subsubsection{Quality of Service}

The demands of delivering high quality of service (QoS) are increasing in RTES designs. QoS is usually associated with the pleasantness of user experience, e.g., the quality of cell phone conversation, or accuracy of control performance, e.g., fuel injection decision to the car engine. Throughout this thesis, the computational requests are modeled as tasks. There exists different QoS models of task executions. In this chapter, we consider the imprecise computation model [LSL ${ }^{+} 94$ ] where the delivered QoS of a task is given as a function of its actual execution time, which can be composed of two parts. The first part is mandatory to be executed, if $\tau_{i}$ is released. This part has a fixed execution efforts $\mathcal{E}_{i}^{m}$ from which the actual execution time can be calculated based on concrete processor frequencies. The second part is optional with a variable execution efforts $c_{i}^{o}$ that is in the range $\left[0, \mathcal{E}_{i}^{o}\right]$. The upper bound of execution $\mathcal{E}_{i}^{o}$ can be 0 , indicating that there is no additional execution associated with $\tau_{i}$. Similarly, the actual execution time of the optional part at a given frequency can also be derived correspondingly from $c_{i}^{o}$. 
The mandatory part delivers a constant QoS value, $\mathcal{Q}_{i}^{m}$, that task $\tau_{i}$ delivers minimally. The optional part leads to additional QoS depending on the actual extra execution time, $c_{i}^{o}$, allocated. We would like to emphasize that only the first, mandatory, part is obligatory for each task. Thus, some tasks may not have any optional part, which means that the quality of service produced by these tasks, at each activation, will be $\mathcal{Q}_{i}^{m}$. For the other tasks with optional parts, the more execution is performed, the higher QoS the task produces. Then the QoS value produced by $\tau_{i}$ as a whole is as follows,

$$
Q o S_{i}=\mathcal{Q}_{i}^{m}+\mathcal{F}_{i}\left(c_{i}^{o}\right),
$$

where $\mathcal{F}_{i}$ is the non-decreasing reward function of task $\tau_{i}$. Thereby, the total QoS delivered by the system in mode $M$ is calculated as

$$
Q_{o} S_{M}=\sum_{\tau_{i} \in M} Q o S_{i}
$$

\subsubsection{Quality of Confidentiality}

Let us denote the $j$-th message generated by task $\tau_{i}$ as $m_{i j}$. In this chapter, we shall assume the use of RC6 (refer to Section 3.2.1.1) due to its wide range of strength/overhead trade-off. Assuming that the RC6 variant applied on $m_{i j}$ is $C_{i j}$, then the quality of confidentiality (QoC) protection for $m_{i j}$ is quantified as

$$
Q o C_{i j}=\frac{e^{\operatorname{Strength}\left(C_{i j}\right) / M A X}-1}{e-1},
$$

where, $M A X$ is the highest protection strength value required in the system, e.g. 118 delivered by RC6-16 in Table 3.1. The set of all messages over which task $\tau_{i}$ interacts with the environment is captured in $\mathcal{L}_{i}$. The weight representing the relative importance of message $m_{i j}$ is denoted as $w_{i j}$. Now we can define the QoC delivered by the system in a mode $M$ as follows,

$$
Q \circ C_{M}=\frac{\sum_{\tau_{i} \in M} \sum_{m_{i j} \in \mathcal{L}_{i}} Q o C_{i j} \cdot w_{i j}}{\sum_{\tau_{i} \in M} \sum_{m_{i j} \in \mathcal{L}_{i}} w_{i j}} .
$$

When the system is switching into another mode and the IBC selection for a message is to be changed, the system will send out an 
encrypted notification message to the corresponding communication peer to notify the update. This is carried out only when the requirement changes and the IBC selection is also changed. The extra time overhead of generating and transmitting the notification messages is very small compared to the overall computation and communication load due to normal functionality. Therefore, we did not explicitly model this process.

\subsubsection{Intrusion Detection Accuracy}

Another design objective is to maximize the intrusion detection accuracy. As described in Section 3.2.2, we quantify the accuracy as $I D A=\frac{\mathcal{P}_{\mathcal{I D}}^{\min }}{\mathcal{P}_{I D}}$ (Equation 3.11). The ID task has two associated parameters, i.e., a constant $\mathrm{ET}, \mathcal{E}_{I D}$, and the minimal required release interval $\mathcal{P}_{I D}^{\min }$, namely the minimal period, that ID task executes with, in order to achieve the highest detection accuracy. The actual execution time of the ID task is then

$$
c_{I D}\left(f_{I D}\right)=\frac{\mathcal{E}_{I D} \cdot f^{\max }}{f_{I D}},
$$

where $f_{I D}$ is the assigned frequency to the ID task. We assume $\mathcal{D}_{I D}=$ $\mathcal{P}_{I D}$ (the deadline is equal with its current period). Then, we can calculate the processor utilization of the ID task as follows,

$$
U_{I D}=\frac{c_{I D}\left(f_{I D}\right)}{\mathcal{P}_{I D}}
$$

\subsubsection{Average Power Consumption}

The last design goal is to reduce the overall average power consumption (APC) of the system for the mode. Knowing all the components of microprocessor power consumption (see Section 3.1.2), we can calculate its average power consumption by considering the utilization of all the tasks, since the dynamic power is consumed only when a task is actively running on the processor. The dynamic power consumption caused by execution of task $\tau_{i}$ is as follows,

$$
\operatorname{Pow}_{i}^{\text {Dyn }}=C_{e f f_{i}} V_{d d i}^{2} f_{i} .
$$


Correspondingly, we have the dynamic power consumption of the ID task as follows,

$$
\operatorname{Pow}_{I D}^{\text {Dyn }}=C_{\text {eff }}{ }_{I D} V_{d d I D}^{2} f_{I D} .
$$

The power consumed by the E/D operations on $\tau_{i}$ related communication messages is then

$$
\operatorname{Pow}_{\mathcal{L}_{i}}^{D y n}=C_{\text {eff }}^{\text {ED }}{ } V_{d d i}^{2} f_{i} .
$$

$C_{\text {eff ED }}$ is the switching capacitance of the chosen IBC algorithm on the target processor. The E/D operations share the same voltage and frequency with $\tau_{i}$. All the three power consumptions above only occur when the corresponding tasks are actively running on the processor. Now we can calculate the contribution of task $\tau_{i}$ to the APC in mode $M$ as

$$
\begin{aligned}
\text { Pow }_{i} & =\text { Pow }_{i}^{\text {Dyn }} \cdot \frac{c_{i}\left(f_{i}\right)}{\mathcal{P}_{i}}+\operatorname{Pow}_{\mathcal{L}_{i}}^{\text {Dyn }} \cdot \sum_{m_{i j} \in \mathcal{L}_{i}} \frac{c_{i j}^{E D}\left(f_{i}\right)}{\mathcal{P}_{i}} \\
& =V_{d d_{i}}^{2} \cdot\left(C_{e f f_{i}} \cdot \frac{\left(\mathcal{E}_{i}^{m}+c_{i}^{o}\right) \cdot f^{m a x}}{\mathcal{P}_{i}}+\sum_{m_{i j} \in \mathcal{L}_{i}} C_{e f f_{E D}} \cdot \frac{l_{i j} \cdot c_{C_{i j}} \cdot f^{\text {max }}}{\mathcal{P}_{i}}\right) .
\end{aligned}
$$

Similarly, we have the contribution of the ID task as follows,

$$
\begin{aligned}
\text { Pow }_{I D} & =C_{\text {eff }}{ }_{I D} V_{d d I D}^{2} f_{I D} \cdot \frac{c_{I D}\left(f_{I D}\right)}{\mathcal{P}_{I D}} \\
& =V_{d d_{I D}}^{2} \cdot C_{e f f_{I D}} \cdot \frac{\mathcal{E}_{I D} \cdot f^{\text {max }}}{\mathcal{P}_{I D}} .
\end{aligned}
$$

Now we can calculate the APC of the processor in a certain mode $M$ as follows,

$$
\begin{aligned}
\text { Pow }_{M} & =\text { Pow }^{\text {Stat }}+\text { Pow }^{\text {On }}+\text { Pow }^{\text {Dyn }} \\
& =\text { Pow }^{\text {Stat }}+\text { Pow }^{\text {On }}+\text { Pow }_{I D}+\sum_{\tau_{i} \in M} \text { Pow }_{i} .
\end{aligned}
$$

As can be observed from Equation 5.13, Equation 5.14, and Equation 5.15, for a given resource allocation decision (task additional execution times, cipher selection decisions, and ID task period assignment), an effective way to reduce the system average power consumption is to reduce the supply voltages $V_{d d}$ during execution of the 
Table 5.1: Task attributes for Figure 5.1

\begin{tabular}{|c|c|c|c|c|c|c|c|c|c|}
\hline Task & $\mathcal{E}^{m}$ & $\mathcal{E}^{o}$ & $\mathcal{P}=\mathcal{D}$ & $\mathcal{L}$ & $C_{\text {eff }}$ & $\mathcal{Q}^{m}$ & $\alpha$ & $\beta$ & $\gamma$ \\
\hline$\tau_{1}$ & 300 & 400 & 1500 & $\left\{m_{11}\right\}$ & 8 & 4 & 0.008 & 0.1 & 0.08 \\
\hline$\tau_{2}$ & 400 & 600 & 2300 & $\emptyset$ & 5 & 5 & 0.016 & 0.13 & 0.09 \\
\hline$\tau_{3}$ & 600 & 300 & 2500 & $\left\{m_{31}\right\}$ & 9 & 7 & 0.009 & 0.9 & 0.17 \\
\hline$\tau_{4}$ & 500 & 700 & 2000 & $\left\{m_{41}\right\}$ & 6 & 4 & 0.011 & 0.08 & 0.12 \\
\hline
\end{tabular}

tasks, using the dynamic voltage and frequency scaling (DVFS) technique. Recent works $\left[\mathrm{PPS}^{+} 13, \mathrm{HMGM} 13, \mathrm{LPD}^{+} 14\right]$ have shown that, for modern microprocessors, the switching between voltages (and frequencies) is performed very efficiently with overheads at the scale of $\mu s$ (time) and $\mu J$ (energy). Such overheads are negligible compared with the duration and energy consumption of the actual applications. Therefore, we ignore the overhead due to switching between voltages, and focus on the overall power consumption of the application.

\subsection{Motivational Example}

Now let us consider the system illustrated in Figure 5.1 with given task parameters in Table 5.1. The reward functions of all tasks are set to have the form $Q o S_{i}=\mathcal{Q}_{i}^{m}+\alpha \cdot c_{i}^{o}+\beta \cdot \sqrt{c_{i}^{o}}+\gamma \cdot \sqrt[3]{c_{i}^{o}}$. Each task is associated with at most one message for illustration purposes. The length and importance weights $(l, w)$ of messages $m_{11}, m_{31}$, and $m_{41}$ are $(2,0.7),(3,0.3)$, and $(1,0.4)$, respectively. The processor supports 7 discrete supply voltage levels that are $1.2 \mathrm{~V}, 1.3 \mathrm{~V}, \ldots, 1.8 \mathrm{~V}$. Note that the value of the nominal task execution times are given according to the highest supply voltage $V d d^{\max }=1.8 \mathrm{~V}$, i.e., the highest frequency $f^{\max }=777 \mathrm{MHz}$, thus, are the shortest time values. We assume that the ID task takes 400 time units to finish a detection procedure at $f^{\max }$, and delivers the best protection with period $\mathcal{P}_{I D}^{\min }=1500$ time units (refer to Section 5.2.3).

Let us look at the case when the system is going to switch to mode $M^{134}=\left\{\tau_{1}, \tau_{3}, \tau_{4}\right\}$, in which task $\tau_{2}$ is not active. With the 
given attributes in Table 5.1, we can calculate the smallest processor utilization, that is, when the system only carries out the mandatory parts of $\tau_{1}, \tau_{3}, \tau_{4}$, and does not pursue any security protections or extra QoS. We can find that, if the processor runs at the highest frequency, the utilization is $U=\mathcal{E}_{1}^{m} / \mathcal{P}_{1}+\mathcal{E}_{3}^{m} / \mathcal{P}_{3}+\mathcal{E}_{4}^{m} / \mathcal{P}_{4}=0.69$. This indicates that the processor is underutilized if all the tasks are executed at $V d d=1.8 \mathrm{~V}$. Therefore, in order to improve the power efficiency of the system, we can lower the processor supply voltage until the system schedulability cannot be guaranteed any longer. If all tasks share the same voltage level, then the lowest voltage that the system can operate at correctly with executing only the mandatory parts of the tasks is $V d d=1.4 V(f=554 \mathrm{MHz})$. However, this is not the most power efficient solution $\left(\right.$ Pow $\left._{M^{134}}=0.93 W\right)$. If we assign $1.4 \mathrm{~V}, 1.3 \mathrm{~V}$, and $1.5 \mathrm{~V}$ to $\tau_{1}, \tau_{3}$, and $\tau_{4}$, respectively, then we can reach the lowest power consumption of Pow $_{M^{134}}^{\prime}=0.92 \mathrm{~W}$, which is the optimal power for mode $M^{134}$ under the current setting. Note that the system provides minimal QoS, and does not carry out any security protections with this configuration.

If we want to maximize the delivered QoS, we would like to provide the maximal execution time to the optional parts, i.e., $c_{1}^{o}=400$, $c_{3}^{o}=300$, and $c_{4}^{o}=700$. However, this cannot lead to a feasible solution even if we set the supply voltage to the maximal value $V d d=$ $1.8 \mathrm{~V}$ for all the three tasks. In fact, the highest achievable QoS $\left(Q o S_{M}=41.51\right)$ can be obtained by having $c_{1}^{o}=30.15, c_{3}^{o}=300$, and $c_{4}^{o}=339.46$. And the supply voltages are all set to $V d d=$ $1.8 \mathrm{~V}$ to ensure system schedulability. Consequently, the system power consumption is $\operatorname{Pow}_{M^{134}}=2.06 \mathrm{~W}$. However, there is absolutely no security protection in this case, since the extra computation efforts are all dedicated to improve the QoS performance, and no message $\mathrm{E} / \mathrm{D}$ or ID operation is performed.

Alternatively, we can protect the system communication, namely the messages $m_{11}, m_{31}$, and $m_{41}$, with the highest confidentiality protection $\left(Q o C_{M}=1\right)$, that is to encrypt them with the strongest cipher variant available, e.g., as RC6-16 from Table 3.1. Thereby, we need to devote 140,210 , and 70 time units (at $V d d=1.8 V$ ) on encrypting $m_{11}, m_{31}$, and $m_{41}$, respectively. Consequently, we have 
the power consumption of $P_{0} w_{M}^{134}=2.07 \mathrm{~W}$. In fact, the condition $Q o C_{M}=1$ can be reached with the optimal power efficiency by setting $V d d_{1}=1.7 \mathrm{~V}, V d d_{3}=1.8 \mathrm{~V}$, and $V d d_{4}=1.6 \mathrm{~V}$ that lead to Pow $_{M^{134}}=1.89 \mathrm{~W}$. In the same way, the highest ID level $(I D A=1)$ can also be independently optimized, i.e., to carry out the ID task with period $\mathcal{P}_{I D}=\mathcal{P}_{I D}^{\min }=1500$. To this end, the most power efficient solution can be reached by assigning $V d d_{1}=1.8 \mathrm{~V}, V d d_{3}=1.6 \mathrm{~V}$, $V d d_{4}=1.8 \mathrm{~V}$, and $V d d_{I D}=1.8 \mathrm{~V}$, which give $\operatorname{Pow}_{M^{134}}=1.84 \mathrm{~W}$.

The four design scenarios outlined above (to independently minimize system power consumption, or maximize QoS, QoC, or IDA) represent cases in which the system either only undertakes the mandatory computations or ignores extra QoS or other security demands. In practice, none of these extreme corner cases may be attractive. Instead, the designer may prefer to achieve a reasonable balance among the four objectives, which makes the underlying design problem for each mode $M$ a multi-objective optimization problem along these four dimensions. The solutions to this problem can be captured in a $4 \mathrm{D}$ space having infinite amount of points. However, due to the time and memory limitations, we cannot afford to find and store all the optimal points. Thereby, in the rest of this chapter, we refer to a subset of finite near-optimal points from the solution space as the Pareto Space $S_{M}$ of mode $M$. In other words, $S_{M}$ represents the set of solutions that we find and store in memory. The points on $S_{M}$ cannot dominate each other, that is, be better in all design dimensions, and are considered to be equally good but with different emphasizes.

A solution $s \in S_{M}$ consists of the resource allocation decisions (processor bandwidth and voltage selections) for the four objectives, i.e., a certain delivered QoS (as result of the allocated optional execution time to application tasks), QoC (as result of the cipher variant assignments), IDA (as result of the chosen period of the ID task), and APC (as result of the allocated processor bandwidth and voltages to the tasks). For example, the Pareto space for mode $M^{134}$ is depicted in Figure 5.3. For illustration purposes, we try to plot the 4D Pareto space within a 3D dimension. Thereby, we denote the fourth design objective, i.e., the average power consumption, using the color-map shown on the right side of the plot. If a point is more towards the 


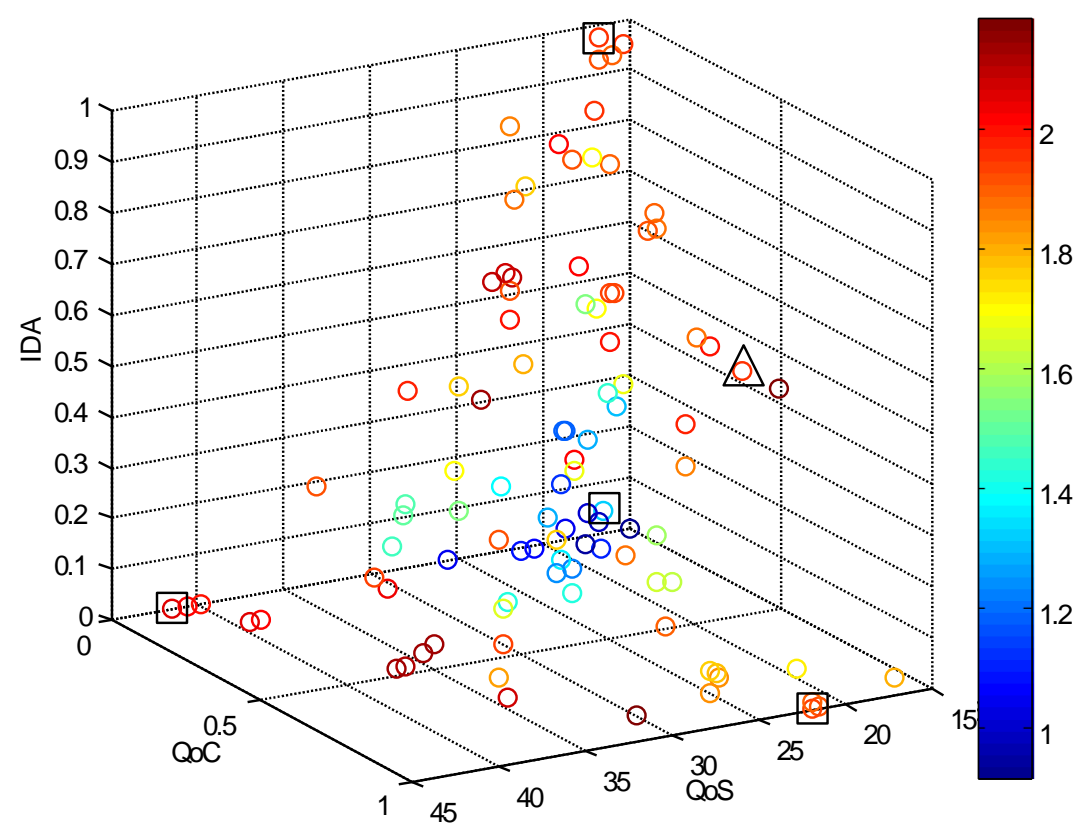

Figure 5.3: Pareto space of mode $M^{134}$

red (or blue), then it implies higher (or lower) power consumption. The solutions to the four aforementioned corner cases are highlighted with squares.

Let us assume that, during run-time, the points shown in the Pareto space in Figure 5.3 are stored in memory, and the system switches to mode $M^{134}$. The question at this moment is how to distribute the processor bandwidth among execution demands, e.g., how much to provide to security services and at which voltage level to run the tasks. Based on the actual system status and external threat level, the security monitor or remote control center determines the security requirement levels as $Q o C^{R}$ and $I D A^{R}$ for confidentiality and intrusion detection, respectively. The system power manager retrieves the current battery information, and sets the maximal power budget $P o w^{R}$. With the constraints $Q o C^{R}, I D A^{R}$, and $P o w^{R}$, the run-time manager can look for an operation point from the Pareto space that satisfies all constraints, and produces the highest QoS. This procedure is performed every time the system is entering a new mode, or any of 


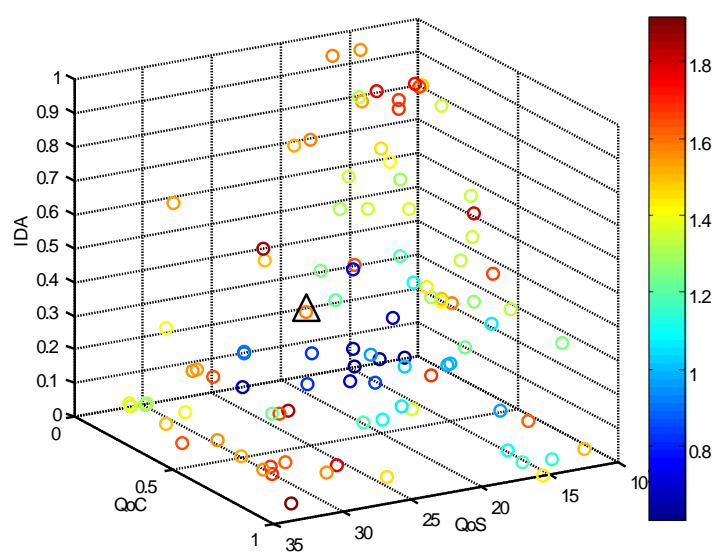

Figure 5.4: Derived solution space for mode $M^{13}$ from $M^{123}$

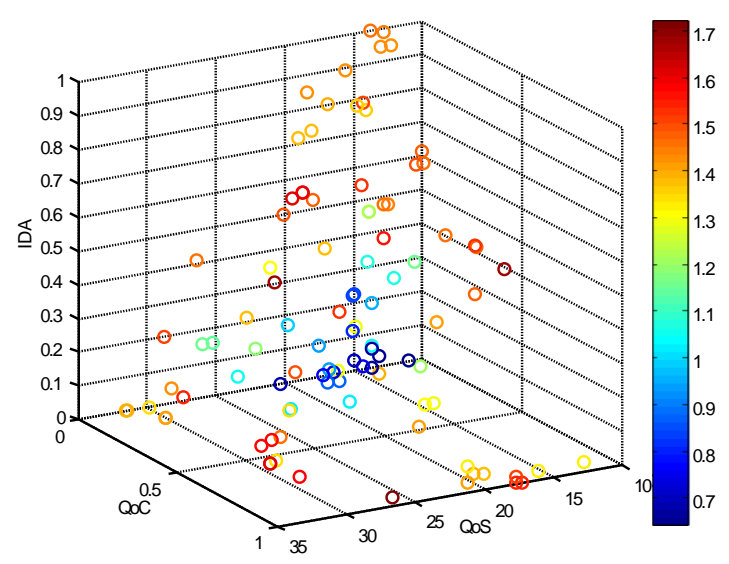

Figure 5.5: Derived solution space for mode $M^{13}$ from $M^{134}$

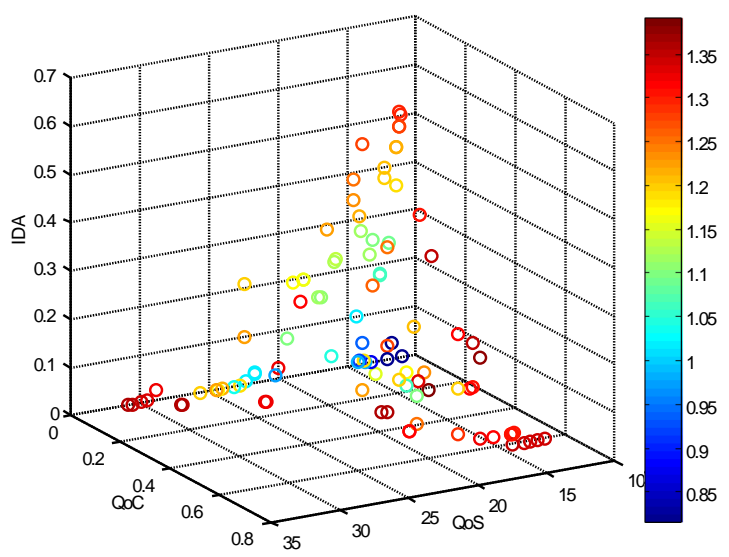

Figure 5.6: Derived solution space for mode $M^{13}$ from $M^{1234}$ 
the system states (e.g., security threats and battery status) change. For example, if the current constraints are $Q o C^{R}=0.7, I D A^{R}=0.5$, and $P_{o w}{ }^{R}=2.2 \mathrm{~W}$, the run-time manager can choose the resource allocation decision specified by the point $(18.8,0.71,0.52,2.19)$ (marked with a triangle in Figure 5.3). In fact, $Q o S=18.8$ is the highest value that can be achieved under the given constraints (security, power, and schedulability). The system configurations for this operation point are as follows, $c_{1}^{o}=3, c_{3}^{o}=8.15, c_{4}^{o}=6.67, C_{11}=$ RC6-12, $C_{31}=$ RC6-12, $C_{41}=$ RC6-12, $\mathcal{P}_{I D}=2881.28, V d d_{1}=1.8, V d d_{3}=1.8$, $V d d_{4}=1.8$, and $V d d_{I D}=1.8$.

If all functional modes have their corresponding Pareto spaces stored in memory, then the above procedure can be repeated when new modes are entered or system states are changed, and allows determining the best operation points based on the current situation, on-the-fly. However, this is only the ideal case, and there are several reasons why this is not possible:

- The number of functional modes to be processed grows exponentially with the increasing number of tasks $|\mathcal{T}|$. Thus, it can be impossible, even at design-time, to explore all functional modes and generate the Pareto spaces accordingly for large systems.

- Solving the multi-objective optimization problem identified earlier in this chapter is of exponential complexity.

- The size of available memory on the target system is limited, thus restricting the number of Pareto spaces that can be stored.

Thus, we can only afford to explore and save a limited amount of Pareto spaces for selected modes. Hence, the next question is, when the system enters a mode which has no Pareto space pre-stored, how a good operation point can be quickly identified. In this chapter, we propose an efficient on-line method that extrapolates an operation point from the available pre-stored spaces.

Let us consider the scenario that the system currently runs in mode $M^{134}$, and must switch to mode $M^{13}$ for which no Pareto space is pre-stored. In this case, the system tries to make use of the available Pareto spaces of its supermodes in order to find a good operation 
point for $M^{13}$. In this example, we assume that the Pareto spaces of modes $M^{123}, M^{134}$, and $M^{1234}$ are available in memory. What is needed is to derive an approximation of the Pareto space for the mode $M^{13}$ as a starting point, from the available Pareto spaces of its supermodes. Since all supermodes in $\overline{\mathcal{M}}\left(M^{13}\right)$ contain task $\tau_{1}$ and $\tau_{3}$ together with other tasks, the system can derive a solution space from the Pareto spaces of $\overline{\mathcal{M}}\left(M^{13}\right)$ by ignoring the utilization due to the non-existing tasks $M^{123} \backslash M^{13}=\left\{\tau_{2}\right\}, M^{134} \backslash M^{13}=\left\{\tau_{4}\right\}$, and $M^{1234} \backslash M^{13}=\left\{\tau_{2}, \tau_{4}\right\}$.

Figure 5.4, 5.5, and 5.6 depict the derived spaces for $M^{13}$ from its supermodes $M^{123}, M^{134}$, and $M^{1234}$, that are denoted as $S_{13}^{123}, S_{13}^{134}$, and $S_{13}^{1234}$, respectively. The next step is to look for a good operation point from the derived spaces for $M^{13}$ based on concrete operational requirements. Assuming that the current constraints are $Q o C^{R}=0.5$, $I D A^{R}=0.4$, and $P o w^{R}=2$, then we can look for the most promising operation point, i.e., that meets all the constraints and delivers the highest QoS value, directly from the derived spaces. In this case, we come to an operation point $s=(25.64,0.52,0.41,1.57)$ on the derived spaces $S_{13}^{123}$ (as marked in Figure 5.4 with a triangle). The corresponding system parameters are set as $c_{1}^{o}=0.48, c_{3}^{o}=176.74$, $C_{11}=\mathrm{RC} 6-8, C_{31}=\mathrm{RC} 6-12, \mathcal{P}_{I D}=3682.63, V d d_{1}=1.7, V d d_{3}=$ 1.7 , and $V d d_{I D}=1.7$. Let us remind that, this point $s$ is obtained by ignoring the computational resources allocated for task $\tau_{2} \in M^{123}$ that does not exist in mode $M^{13}$. Therefore, this solution may be sub-optimal, since a portion of the processor bandwidth is left unused. The current processor utilization with respect to this selected point is only $U=0.79$, and the power consumption is Pow $=1.57$. Therefore, we can further improve the QoS by extending, in the next step, the optional execution time of $\tau_{1}$ and $\tau_{3}$ to $c_{1}^{o}=225.75$ and $c_{3}^{o}=300$, respectively. By this, the system QoS is increased to $Q o S=34.22$ accordingly, and all operational constraints are still met. More details regarding the above procedure will be discussed in Section 5.5. 


\subsection{Problem Formulation}

We formulate the design problem into a two-stage optimization. At design-time, we prepare Pareto spaces for the system to use at runtime. This is captured as a multi-objective optimization problem on all functional modes. There are potentially $|\mathcal{M}|=O\left(2^{|\mathcal{T}|}\right)$ instances of optimization problems to be solved, which becomes infeasible to go through as $|\mathcal{T}|$ grows. Thus, we can only afford to explore a subset of $\mathcal{M}$ limited by the available off-line design optimization time. Then, depending on the size of available memory of the target platform, we select the Pareto spaces of the most rewarding modes to be stored in the memory. At run-time, the system chooses the best operation solution whenever needed, e.g., when the system is switching to another mode or the current security demands or available power budget is changed.

\subsubsection{Design-Time Optimization}

For each functional mode, we would like to find system designs considering four objectives, i.e. QoS (Equation 5.5), QoC (Equation 5.7), IDA (Equation 3.11), and APC (Equation 5.15). As already mentioned, the solutions to this problem can be represented by a $4 \mathrm{D}$ Pareto space. Theoretically, there are infinite number of operation points in this space, but we only can explore and store a finite number of them due to time and space limitations. Before going further, let us first introduce the method that we used to compare two solution spaces $S_{i}$ and $S_{j}$. We say that space $S_{i}$ outperforms $S_{j}$ if and only if the hypervolume [ZT98] $\mathcal{H}$ of $S_{i}$ is strictly larger than that of $S_{j} ; \mathcal{H}\left(S_{i}\right)>\mathcal{H}\left(S_{j}\right)$. The hypervolume is the volume of the objective space under the surface defined by the dominating points that represents the quality of a solution space. The calculation of hypervolume is performed according to the algorithm introduced in [Zit01]. Thereafter, we also define the hypervolume of a mode $M$. If $S_{M}$ is stored in memory, i.e., $M \in \mathcal{M}^{\text {mem }}\left(\mathcal{M}^{\text {mem }}\right.$ is the set of modes which have their Pareto spaces stored in memory), then $\mathcal{H}_{M}$ is the hypervolume $\mathcal{H}\left(S_{M}\right)$. Otherwise, $\mathcal{H}_{M}$ is defined as the hypervolume of the best derived space from its supermodes. Then the hypervolume of mode 
$M$ given the pre-stored Pareto spaces $\mathcal{M}^{\text {mem }}$ is calculated as

$$
\mathcal{H}_{M}\left(\mathcal{M}^{m e m}\right)=\left\{\begin{array}{l}
\mathcal{H}\left(S_{M}\right), \text { if } M \in \mathcal{M}^{m e m} \\
\max _{M^{\prime} \in\left(\overline{\mathcal{M}}(M) \cap \mathcal{M}^{m e m}\right)}\left(\mathcal{H}\left(S_{M}^{M^{\prime}}\right)\right), \text { otherwise. }
\end{array}\right.
$$

$S_{M}^{M^{\prime}}$ is derived for $M$ from its supermode $M^{\prime}$ by removing the resources occupied by tasks $M^{\prime} \backslash M$. That is, $S_{M}^{M^{\prime}}$ collects a set of solutions for mode $M$ which are obtained from the Pareto space of its supermode $S_{M^{\prime}}$ by ignoring the non-existing tasks. This means that $S_{M}^{M^{\prime}}$ is not the real Pareto space for $M$, but a space derived from its supermode, for example, the derived space $S_{13}^{123}$ in Figure 5.4.

We decouple the design-time optimization into three subproblems. The first subproblem is the multi-objective optimization for a given mode $M$. The second subproblem is the global design exploration of the Hasse diagram to obtain the subset $\mathcal{M}^{\text {impl }} \subseteq \mathcal{M}$ containing the candidate modes possibly to be stored in memory. The third subproblem is to select the most rewarding subset $\mathcal{M}^{\text {mem }} \subseteq \mathcal{M}^{\text {impl }}$ to be stored in memory depending on available memory space on the target system.

\subsubsection{Multi-Objective Optimization for A Given Mode}

The inputs to this subproblem are:

- the active tasks in mode $M$;

- the task attributes $\left(\mathcal{E}_{i}^{m}, \mathcal{E}_{i}^{o}, \mathcal{P}_{i}, \mathcal{D}_{i}, \mathcal{L}_{i}, C_{\text {effi }}, \mathcal{Q}_{i}^{m}, \mathcal{F}_{i}\right)$ for all $\tau_{i} \in M$, as well as $l_{i j}, w_{i j}$, and $Q_{m_{i j}}^{m i n}$ corresponding to all messages $m_{i j} \in \mathcal{L}_{i}$;

- execution time $\mathcal{E}_{I D}$ and the optimal period $\mathcal{P}_{I D}^{\min }$ (for achieving full accuracy) for the ID task;

- a strength/time trade-off table of selected cryptographic algorithms and variants (as Table 3.1).

The optimization constraint is the schedulability requirement as described in Equation 5.3, and must be satisfied in all solutions $s \in S_{M}$. 
The optimization objectives are

$$
\begin{aligned}
& \max Q o S_{M} \\
& \max Q o C_{M} \\
& \max I D A_{M} \\
& \min \operatorname{Pow}_{M}
\end{aligned}
$$

And the final output is a Pareto space $S_{M}$ with non-dominated solutions optimized for the four aforementioned objectives.

\subsubsection{Hasse Diagram Exploration}

In this subproblem, the Hasse diagram (see Section 5.1.2.2) capturing the whole set of modes is explored in order to find a subset of candidate modes $\mathcal{M}^{i m p l} \subseteq \mathcal{M}$ to be stored in memory. The inputs to this subproblem are

- the whole set of modes $\mathcal{M}$ modeled as a Hasse diagram;

- the set of functional modes $\mathcal{M}^{\text {func }}$ that may occur at run-time;

- the top functional modes $\mathcal{M}_{\uparrow}^{\text {func }} \subseteq \mathcal{M}^{\text {func }}$ with no functional supermode.

The output for this subproblem is the implementations ${ }^{1}$ of selected modes $\left\{S_{M, \forall M \in \mathcal{M}^{i m p l}}: \mathcal{M}^{i m p l} \subseteq \mathcal{M}^{\text {func }}\right\}$. The constraints are

- each functional mode $M \in \mathcal{M}^{\text {func }}$ must have its own implementation or an implementation of at least one of its supermodes to ensure functionality correctness, i.e.

$$
\forall M \in \mathcal{M}^{\text {func }},(\{M\} \cup \overline{\mathcal{M}}(M)) \cap \mathcal{M}^{i m p l} \neq \emptyset .
$$

This implies the requirement that the top functional modes must be implemented, i.e.,

$$
\forall M \in \mathcal{M}_{\uparrow}^{f u n c}, M \in \mathcal{M}^{\text {impl }} ;
$$

\footnotetext{
${ }^{1}$ We refer to the Pareto space of a mode as its implementation.
} 
- the available optimization time.

The design objective for this problem is to select a set of candidate modes $\mathcal{M}^{i m p l}$ such that the total hypervolume $H$ of all functional modes is maximized, i.e.

$$
\max H=\sum_{M \in \mathcal{M}^{f u n c}} \mathcal{H}_{M}\left(\mathcal{M}^{i m p l}\right),
$$

where, $\mathcal{H}_{M}$ can be calculated according to Equation 5.16 depending on whether $M \in \mathcal{M}^{i m p l}$ or not. Note that, in order to be consistent among the objectives and to correctly calculate the hypervolumes, we transfer the fourth objective of minimize Pow $_{M}$ to maximize the power saving Pow $_{M}^{\text {saving }}$ with respect to a given power budget.

\subsubsection{Selection of Candidate Modes}

In this subproblem, a subset of $\mathcal{M}^{i m p l}$ is identified such that the corresponding set of Pareto spaces is the most rewarding to be stored, at run-time, in memory. The inputs to this subproblem are

- the top functional modes $\mathcal{M}_{\uparrow}^{\text {func }}$ that must have their Pareto spaces in memory;

- the candidate modes $\mathcal{M}^{i m p l}$ and corresponding hypervolumes returned from subproblem 5.4.1.2;

- required memory size for storing each mode $M \in \mathcal{M}^{i m p l}$;

- the available memory space, $\mathrm{Mem}$, on the target system.

The output is the set of chosen modes $\mathcal{M}^{\text {mem }}$, for which the corresponding Pareto spaces are stored in memory, which are later used at run-time. The constraint is that the total memory size of storing $\left\{S_{M}: M \in \mathcal{M}^{m e m}\right\}$ cannot exceed the total size of available memory space $M e m$ on the target system.

\subsubsection{Run-Time Optimization}

At run-time, the system is expected to function with the best configuration, regardless of which mode the system is running on or what 
concrete constraints, e.g., security and power, the system has. This is achieved by realizing a solution selection mechanism which, whenever the system changes behavior (mode or/and constraints), finds an efficient operation point based on the pre-stored Pareto spaces. This problem takes the following as input,

- available Pareto spaces $\left\{S_{M}: M \in \mathcal{M}^{m e m}\right\}$ that are stored in memory;

- the mode $M$ that the system will run in;

- the security constraints, i.e, $Q o C^{R}$ and $I D A^{R}$, received from the run-time security monitor or external control center;

- the maximal power budget $P o w^{R}$.

The constraints are that all the security and real-time requirements must be met, i.e.,

$$
\begin{aligned}
Q_{o} C_{s} & \geq Q_{o} C^{R} \\
I D A_{s} & \geq I D A^{R} \\
P o w_{s} & \leq P o w^{R} \\
U_{s} & \leq 1 .
\end{aligned}
$$

The goal is to find an efficient operation point $s$ based on the prestored Pareto spaces, that delivers as high QoS as possible. If $M \in$ $\mathcal{M}^{m e m}$, then $s$ is directly selected from the stored Pareto space $S_{M}$. Otherwise, $s$ is chosen from one of the derived spaces of the supermodes $M^{\prime} \in\left(\overline{\mathcal{M}}(M) \cap \mathcal{M}^{m e m}\right)$. Moreover, the free resource released due to non-existing tasks (tasks in $\mathcal{M}^{\prime} \backslash \mathcal{M}$ ) should be further distributed to maximize QoS.

\subsection{Proposed Techniques}

In this section, we shall elaborate on our proposed framework for solving the design optimization problem formulated in the previous section. We present the overall flow diagram of our design framework in Figure 5.7, which, as can be observed, is divided into two phases, an off-line (Section 5.5.1) and an on-line phase (Section 5.5.2). 


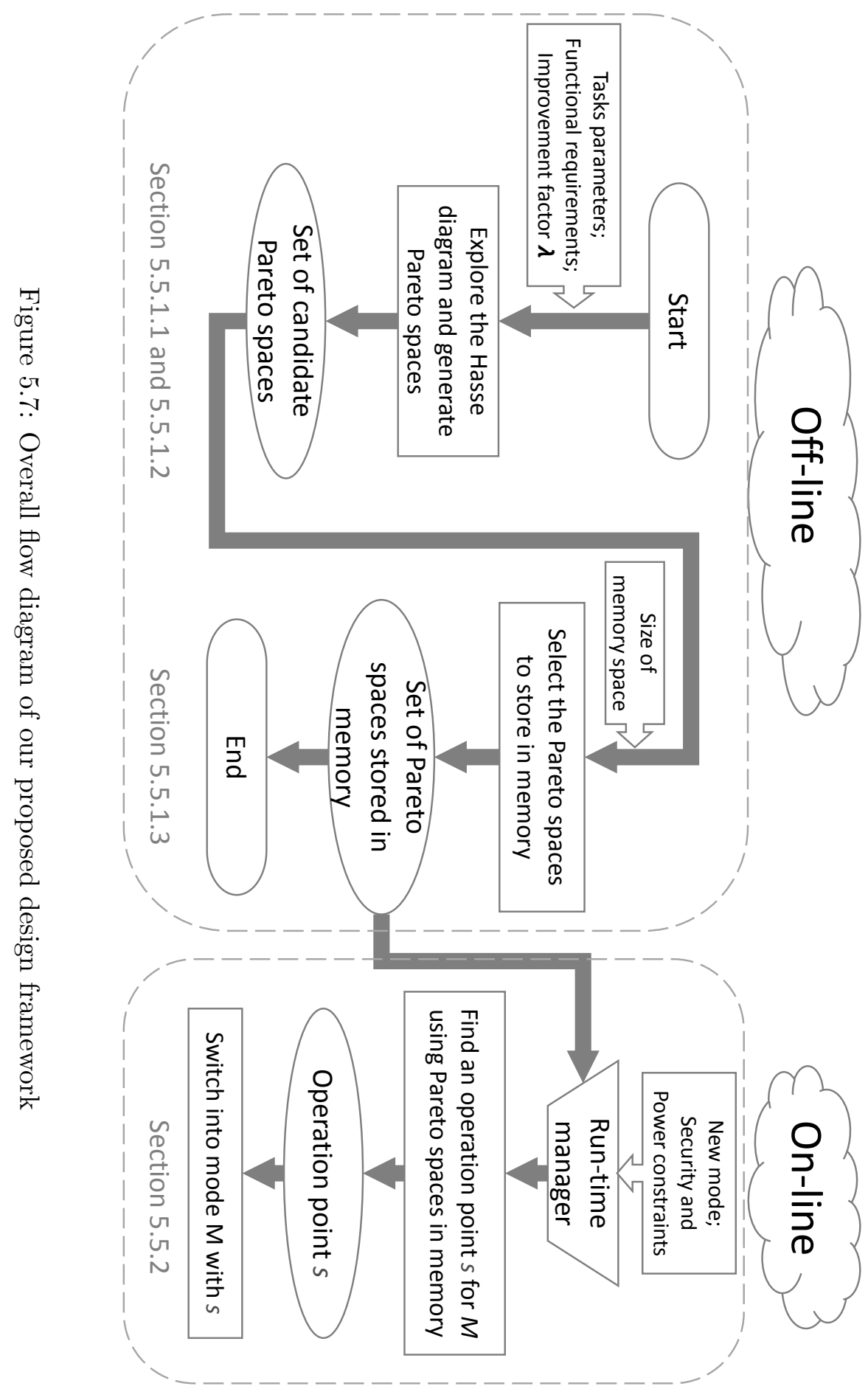




\subsubsection{Design-Time (Off-line Phase)}

\subsubsection{Multi-Objective Optimization for A Given Mode}

As described in Section 5.4.1.1, the system is expected to be optimized along four independent dimensions. However, it is impossible to find the optimal Pareto space even for one given mode, due to the huge computational complexity. Hence, we use a heuristic for generating a near-optimal Pareto space. We chose the genetic algorithm based multi-objective optimization framework NSGA-II [DPAM02] to solve the problem. NSGA-II mimics the process of natural selection via inheritance, mutation, selection, and crossover operations. We packed the following optimization variables as the gene of each individual:

- the additional execution time of each active task;

- the selected cipher or cipher variant on each communication message;

- the period of the ID task $\mathcal{P}_{I D}$;

- the assigned voltage $V_{d d}$ to each task.

Note that, we consider a discrete set of possible periods of the ID task and discrete supply voltages (and frequencies) to the processor.

After a user specified stopping condition is reached, NSGA-II returns a set of solutions that cannot dominate each other on all objectives, and represent the generated Pareto space $S_{M}$ for mode $M$. The parameters used in NSGA-II, e.g. number of generations, population size, and mutation rates, are fine-tuned for different problem sizes.

\subsubsection{Hasse Diagram Exploration}

The main challenge of solving the second subproblem is to efficiently explore the set of functional modes $\mathcal{M}^{\text {func }}$ within the given time and space budget, especially when the total number of tasks $|\mathcal{T}|$ and, thus, number of modes are large. Our proposed technique for approaching the problem is based on a breadth-first search of the Hasse diagram. The modes are explored in a top-down fashion and from the levels of modes with more tasks to the ones with less tasks. Exploration on 
a branch is stopped when there is no significant improvement from saving the Pareto space of a mode over using the derived spaces from its supermodes. This depth of exploration is determined by a user given variable $\lambda$, referred to as the improvement factor, that can be tuned by the designer to trade-off design-time against result quality. Therefore, instead of giving a design-time constraint explicitly, the user provides $\lambda$ as an input to the optimization problem to guide the trade-off. Before going further, let us introduce the definition of immediate submodes of $M$ as

$$
\mathcal{M}^{-}(M)=\left\{M^{\prime} \in \underline{\mathcal{M}}(M):\left|M^{\prime}\right|=|M|-1\right\} .
$$

More precisely, mode $M^{\prime}$ is an immediate submode of mode $M$, if $M^{\prime}$ is a submode of $M$, and has strictly one less task than $M$. For example, $M^{1}=\left\{\tau_{1}\right\}$ is an immediate submode of $M^{12}=\left\{\tau_{1}, \tau_{2}\right\}$, but not of $M^{123}=\left\{\tau_{1}, \tau_{2}, \tau_{3}\right\}$; thus, $M^{1} \in \mathcal{M}^{-}\left(M^{12}\right)$ and $M^{1} \notin$ $\mathcal{M}^{-}\left(M^{123}\right)$.

The main optimization procedure is presented in Algorithm 4. We first define three sets of modes, $\mathcal{M}^{\text {wait }}, \mathcal{M}^{\text {impl }}$, and $\mathcal{M}^{\text {skip }}$, to keep track of the modes that are to be processed, have been implemented, and have been ignored, respectively (Line 1). As already mentioned, the top functional modes $\mathcal{M}_{\uparrow}^{\text {func }}$ must be implemented. Therefore, we first apply NSGA-II on each $M \in \mathcal{M}_{\uparrow}^{\text {func }}$ in order to obtain the corresponding Pareto spaces that must be later stored in memory to guarantee functional correctness. After that, they are included in the implemented mode list $\mathcal{M}^{i m p l}$, and their intermediate submodes are added into the waiting list $\mathcal{M}^{\text {wait }}$ (Line 2-5).

After having produced the Pareto spaces for all $M \in \mathcal{M}_{\uparrow}^{\text {func }}$, we have a set of modes in $\mathcal{M}^{\text {wait }}$ that are to be processed. As long as there still exist modes in $\mathcal{M}^{\text {wait }}$, i.e., $\mathcal{M}^{\text {wait }} \neq \emptyset$, we take out the element from the set that has the most number of tasks $M=$ most_tasks $\left(\mathcal{M}^{\text {wait }}\right)$ as shown in Line 6-7. If $M$ demands to be processed, that is, $M$ is a functional mode, and has not been implemented or skipped, then we derive solution spaces $S_{M}^{M^{\prime}}$ (see Section 5.4.1) for $M$ from all its implemented supermodes $M^{\prime} \in\left(\overline{\mathcal{M}}(M) \cap \mathcal{M}^{i m p l}\right)$, and record the derived space that gives the highest hypervolume $\mathcal{H}_{D}$ (Line 


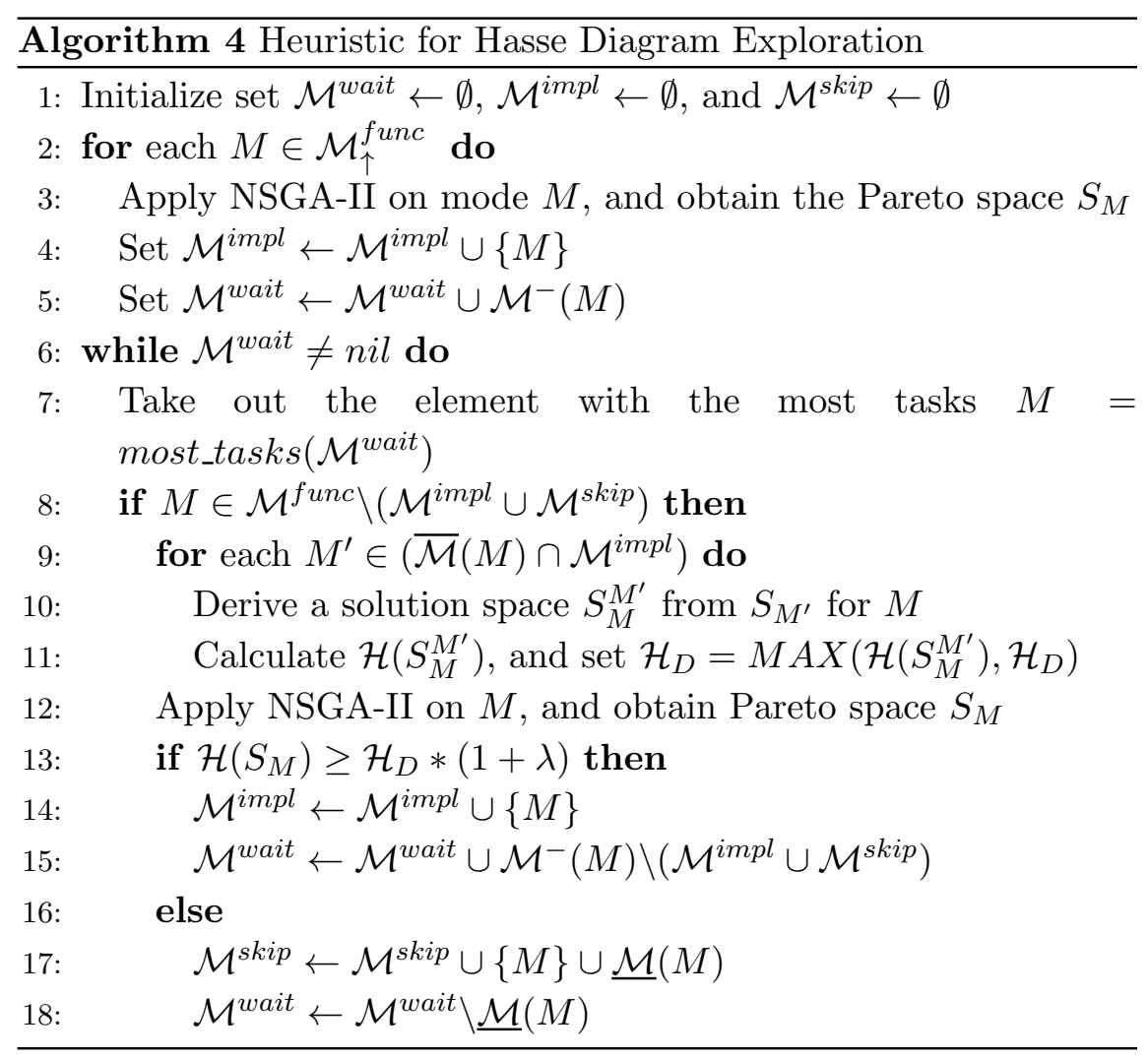

8-11). Thereafter, we apply NSGA-II on $M$ to get the actual space $S_{M}$ as shown in Line 12 .

Now we check whether $S_{M}$ deserves to be implemented and, potentially, stored by comparing the hypervolumes $\mathcal{H}\left(S_{M}\right)$ and $\mathcal{H}_{D}$, that captures how much we gain by having an actual implementation $S_{M}$ as opposed to using the derived spaces at run-time when mode $M$ occurs. We consider that implementing $M$ is sufficiently rewarding, if $\mathcal{H}\left(S_{M}\right) \geq \mathcal{H}_{D} *(1+\lambda)$. If the condition is satisfied, the immediate submodes of $M$, i.e., $\mathcal{M}^{-}(M)$, are added into $\mathcal{M}^{\text {wait }}$ (Line 13-15). Otherwise, the Pareto space of $M$ is discarded, and succeeding searches along its submodes are skipped (Line 16-18). The reason why we decide not to go through the submodes $\underline{\mathcal{M}}(M)$ is that, if $\mathcal{H}_{D} *(1+\lambda)>\mathcal{H}\left(S_{M}\right)$, the derived space is close to the Pareto space (with respect to the improvement factor $\lambda$ ). In other words, all tasks 
in $M$ have already enjoyed adequate resources in the solutions of the supermode $S_{M_{b}}$, in which they have to compete for resources with additional tasks $M_{b} \backslash M$. Moreover, the tasks in the submodes $\underline{\mathcal{M}}(M)$ are subsets of $M$. So they will also receive sufficient resources in the solutions of $S_{M_{b}}$. Therefore, we heuristically skip the submodes $\underline{\mathcal{M}}(M)$ to achieve faster optimization convergence. When the Hasse diagram exploration terminates, that is, $\mathcal{M}^{\text {wait }}=\emptyset$, we have a set of mode implementations $\mathcal{M}^{\text {impl }}$.

The improvement factor $\lambda(\lambda \geq 0)$ in Line 13 is a parameter that the designer can tune for changing the exploration depth, and thereby, trading-off solution quality with optimization time and maximal needed memory. The smaller $\lambda$ is, the more modes on the Hasse diagram are traversed. The most extensive exploration happens when $\lambda=0$, in which case all functional modes are analyzed.

\subsubsection{Selection of Candidate Modes}

After the previous stages, the designer has a set of modes $\mathcal{M}^{i m p l}$ with their generated Pareto spaces that are candidates to be stored in the target platform at run-time. However, the memory space of the system may not be sufficient to accommodate all of them. Thus, the last step of the design-time optimization is to further select the best modes to be stored in memory, depending on the actual memory constraint. We formulate this problem as an integer linear programming (ILP) problem. The expected output is the set of chosen modes $\mathcal{M}^{\text {mem }}$ that have their Pareto spaces stored in memory.

We assign each Pareto space $S_{M: M \in \mathcal{M}^{i m p l}}$ with a binary variable $\phi_{M}$. If $\phi_{M}=1$, mode $M$ will have its Pareto space stored in memory. Otherwise, $S_{M}$ is ignored. For all top functional modes, $\phi_{M}$ is set to 1, i.e., $\forall M \in \mathcal{M}_{\uparrow}^{\text {func }}, \phi_{M}=1$. The required memory size of each Pareto space is determined by the total number of points in the Pareto space obtained from NSGA-II times the number of active tasks in the mode. For example, the Pareto space for mode $M^{134}$ contains 100 operation points (see Section 5.3). Then the required memory space for saving $S_{M^{134}}$ is calculated as $100 \times 3 \times \alpha$. The coefficient $\alpha$ is the size of memory needed for saving all the corresponding system parameters, 
e.g., execution times of optional task executions, cipher selections, and voltage levels, of a task. The optimization goal of this ILP problem is to maximize the total hypervolume of the stored Pareto spaces under the memory constraint, similar to the classic Knapsack problem. We used the IBM ILOG CPLEX Optimization Studio to efficiently solve this ILP problem. As our experiments show (Section 5.6.1), solving this ILP problem takes negligible time compared with the first two subproblems.

\subsubsection{Run-Time (On-line Phase)}

At run-time, the system has a set of Pareto spaces $\left\{S_{M}: M \in \mathcal{M}^{m e m}\right\}$ stored in memory. Thus, the system can react to mode, security requirements, and power budget changes dynamically by switching to an operation point that delivers the highest QoS under the current constraints. There exist two potential scenarios when the system is expected to do an operation switch to mode $M$, namely the Pareto space of $M$ is stored in memory $\left(M \in \mathcal{M}^{m e m}\right)$ or not $\left(M \notin \mathcal{M}^{m e m}\right)$. We shall discuss these two scenarios separately in this section. The overall run-time optimization procedure is presented in Algorithm 5.

\subsubsection{1 $M \in \mathcal{M}^{m e m}$}

In this case, the Pareto space (a set of solutions) for mode $M$ is available in memory. Thereafter, the system can directly select the best operation point $s \in S_{M}$ that delivers the highest QoS with respect to given security and power constraints $Q o C^{R}, I D A^{R}$, and $P o w^{R}$ (Line 1-4). It can be possible that there exists no solution from the space $S_{M}$ satisfying all the constraints, even if the processor is set to the highest supply voltage and no optional task execution is undertaken. If such situation occurs, the run-time monitor will be notified, and emergency measures must be taken, e.g., to carry out only the mandatory executions of the tasks and turn off the communication, or shut down the system (Line 5-6). 


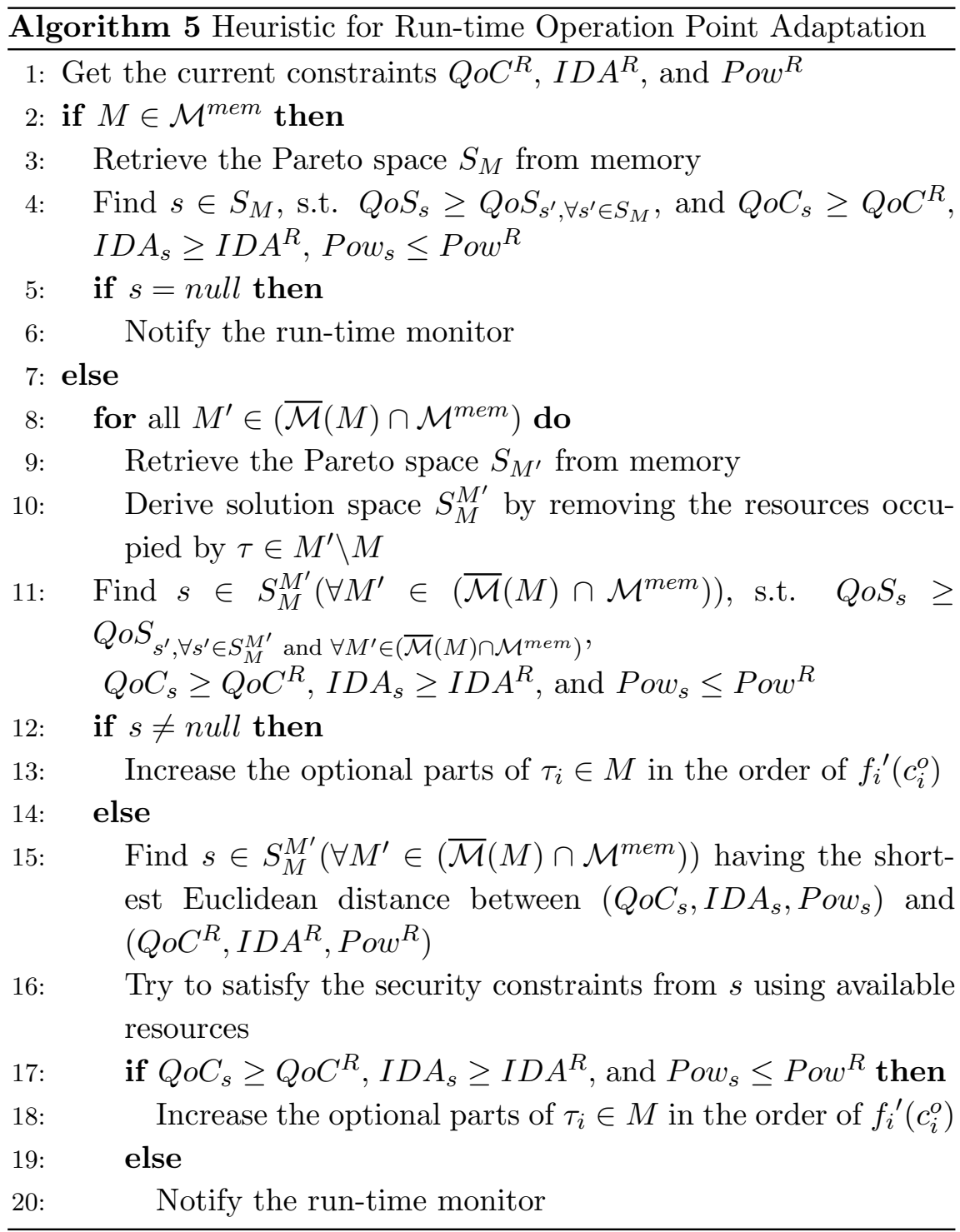

\subsubsection{2 $\quad M \notin \mathcal{M}^{m e m}$}

In this case, we do not have the Pareto space for $M$ stored in memory. Then, the first step is to derive solution spaces from the Pareto spaces of those supermodes of $M$ that are available in the memory, i.e., $M^{\prime} \in$ $\left(\overline{\mathcal{M}}(M) \cap \mathcal{M}^{\text {mem }}\right)$, as shown in Line 8-10. After that, we can look for an appropriate operation point $s \in S_{M}^{M^{\prime}}$ from all the derived spaces 
(Line 11) in which the constraints are satisfied, and the delivered QoS is the highest among all the points. Remember that the solution $s$ is obtained in the way that the resources assigned to tasks $M^{\prime} \backslash M$ are left unused. Therefore, starting from $s$, we can further redistribute the unused resources that were previously occupied by the tasks $M^{\prime} \backslash M$ to enhance system performance. There are two different situations that we must consider depending on whether the constraints can be satisfied with $s$ or not.

If $Q o C^{R}, I D A^{R}$, and Pow ${ }^{R}$ can be satisfied in $S_{M}^{M^{\prime}}$ ( $\left.s \neq n u l l\right)$, we shall get an operation point $s$ from the previous step, and then leave the security protections unchanged. Until now, we have not utilized the free resources that were previously occupied by the non-existing tasks in $M^{\prime} \backslash M$. Thereby, we can further improve the system QoS by increasing the optional task executions among the tasks $\tau_{i} \in M$ in the priority order of the derivative $f_{i}{ }^{\prime}\left(c_{i}^{o}\right)$ of the reward function (refer to Equation 5.4) until the real-time constraint or/and power budget is violated (Line 12-13). By this, resources are provided with priority to those tasks whose QoS deliveries are more sensitive to extra assigned processor bandwidth.

Otherwise $\left(s=\right.$ null), we start from the solution $s \in S_{M}^{M^{\prime}}$ that has the shortest Euclidean distance from $\left(Q o C_{s}, I D A_{s}, P o w_{s}\right)$ to $\left(Q \circ C^{R}, I D A^{R}, P o w^{R}\right)$ (Line 15). We first check whether $I D A_{s} \geq$ $I D A^{R}$ or not. If not, then we allocate unused processor bandwidth to achieve the required period of the ID task $\mathcal{P}_{I D s}=\mathcal{P}_{I D}^{\min } / I D A^{R}$. If the processor utilization bound and power budget are not violated, we continue with a similar procedure regarding the requirement $Q o C^{R}$. If $Q o C^{R}$ is not met, we distribute the currently available resources to improve the confidentiality level to the communication messages with priority to the messages with higher importance $w_{i j}$ (Line 16). In the end, if $P o w^{R}$ as well as $Q o C^{R}$ and $I D A^{R}$ are all satisfied, the same QoS improvement method is carried out as stated in the previous case (Line 17-18). In case any of the requirements $Q o C^{R}, I D A^{R}$, and $P o w^{R}$ cannot be achieved, the run-time monitor will be signaled (Line 19-20), and emergency measures are carried out. 


\subsection{Experimental Results}

We have conducted experiments on a Linux machine having a fourcore Intel Xeon CPU with $2.66 \mathrm{GHz}$ frequency and 8GB RAM. We have studied four different problem sizes having task numbers $|\mathcal{T}|=$ $4,6,8$, and 10 . On each problem size, 20 test applications were randomly generated, in which $80 \%$ of the modes are functional modes. The ID task execution time $\mathcal{E}_{I D}$ and minimal period $\mathcal{P}_{I D}^{\min }$ were set as 400 and 1500 time units. The mandatory execution time $\mathcal{E}_{i}^{m}$ and maximal optional execution time $\mathcal{E}_{i}^{o}$ of task $\tau_{i}$ were randomly chosen from the intervals $[500,1400]$ and $[100,800]$, respectively. Each task was associated with a set of messages, each of which had a length of $l_{i j} \in$ $\{1,2, \ldots, 8\}$ blocks and a random weight $w_{i j} \in[0,1]$. The reward functions were assumed to be of the form $f_{i}\left(c_{i}^{o}\right)=\alpha_{i} \cdot c_{i}^{o}+\phi_{i} \cdot \sqrt{c_{i}^{o}}+\gamma_{i} \cdot \sqrt[3]{c_{i}^{o}}$.

\subsubsection{Design-Time}

We first evaluated our design-time optimization technique. Five different improvement factors $\lambda$ were studied in the experiments, i.e. 0, $0.2,0.4,0.8$, and 1.6 , to evaluate the trade-off between optimization time and performance. The larger $\lambda$ was, the less modes were explored on the Hasse diagram. Thus, less optimization time was required, but with a potential sacrifice in result quality. The situation with $\lambda=0$ provided the most extensive exploration, in which the algorithm exhaustively visited the Hasse diagram, and generated Pareto spaces for all functional modes. We assumed that the available memory in the system was $70 \%$ of the total required memory ${ }^{2}$ of storing $\mathcal{M}^{\text {impl }}$, i.e., $M e m=\operatorname{Mem}\left(\mathcal{M}^{i m p l}\right) * 0.7$.

We have run the off-line step on problem sizes with 4, 6, 8, and 10 tasks. As mentioned earlier, for each of the four levels, we generated 20 instances resulting in a total 80 test sets. The experiments have been run with the five different values of $\lambda$ yielding a total of 400 cases. For each of these cases, a set of Pareto spaces has been obtained to be stored, at run-time, in the memory of the target system.

Let us remind that each functional mode $M \in \mathcal{M}^{\text {func }}$ must have

\footnotetext{
${ }^{2}$ We did not restrict the memory size under $\lambda=0$ for comparison purposes.
} 
its own implementation or an implementation of at least one of its supermodes stored in memory to ensure functional correctness. Therefore, the scenario when the system has only the Pareto spaces of the top functional modes, i.e. $\mathcal{M}^{\text {mem }}=\mathcal{M}_{\uparrow}^{\text {func }}$, is the minimal set of feasible solutions. Under this case, the total hypervolume $H_{\mathcal{M}_{\uparrow}^{f u n c}}$ is actually the minimum that could be obtained satisfying all functional requirements. Thereby, we chose this case as the baseline for comparison in our experiments. For each $\lambda$ on a given test application, we computed the achieved performance improvement $(P I)$ ratio as follows,

$$
P I=\frac{H_{\mathcal{M}^{\text {mem }}}-H_{\mathcal{M}_{\uparrow}^{\text {func }}}}{H_{\mathcal{M}_{\uparrow}^{\text {func }}}} * 100 \%,
$$

where $H_{\mathcal{M}^{\text {mem }}}$ is the total hypervolume of all functional modes under the situation when the Pareto spaces of modes in $\mathcal{M}^{\text {mem }}$ were stored in memory (refer to the cost function in Equation 5.18).

Figure 5.8 shows the obtained results comparing with the baseline solution $\mathcal{M}^{\text {mem }}=\mathcal{M}_{\uparrow}^{\text {func }}$. The horizontal and vertical axes indicate the number of tasks $|\mathcal{T}|$ and the average $P I$ in each experimental setup, respectively. It can be observed that, on the same problem size, bigger improvements can be achieved with smaller $\lambda$, since more modes are explored and stored. For example, with the problem size of 8 tasks, the obtained $P I$ values using $\lambda=0.2$ and $\lambda=1.6$ were $121 \%$ and $38 \%$, respectively. Of course, the general trend shows that, with a smaller $\lambda$, the performance improvements PI are larger, since the number of explored modes increases. However, it is also visible that with a $\lambda$ of, e.g., 0.4, already significant levels of PI can be achieved.

The average optimization time (in seconds) for each $\lambda$ and problem size is depicted in Figure 5.9. As can be noticed, no result was shown for the largest problem size with the smallest improvement factor, i.e., $|\mathcal{T}|=10$ and $\lambda=0$, in Figure 5.8 and 5.9. The reason is that the experiments took extremely long run-times that are beyond our timeout restriction. This reflects the fact that the Hasse diagram exploration with small $\lambda$ (or, in the extreme case, $\lambda=0$ ) induces huge computational cost. It becomes impractical to explore a big portion of the Hasse diagram using a small $\lambda$ for large system designs. However, 


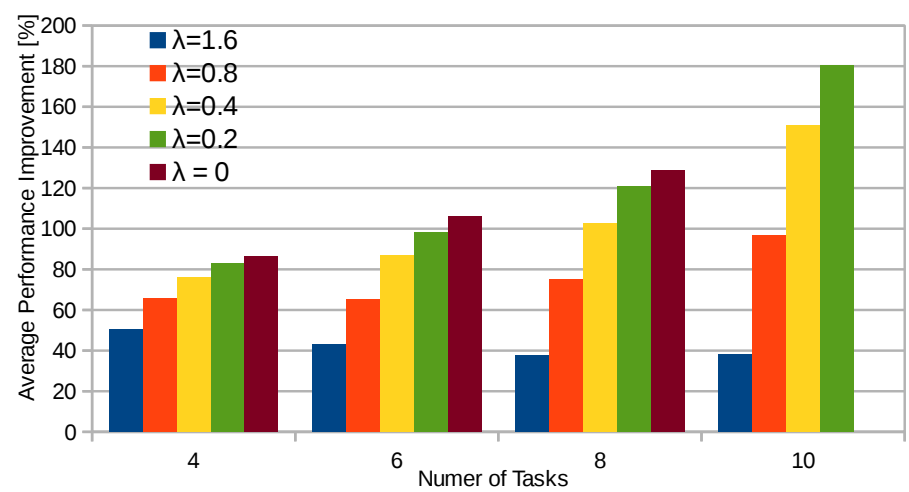

Figure 5.8: Performance improvement of off-line phase

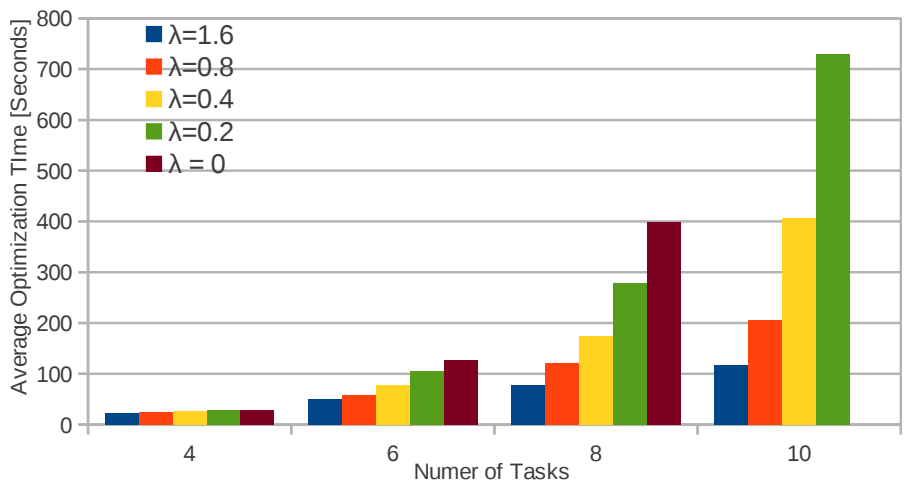

Figure 5.9: Optimization time of off-line phase

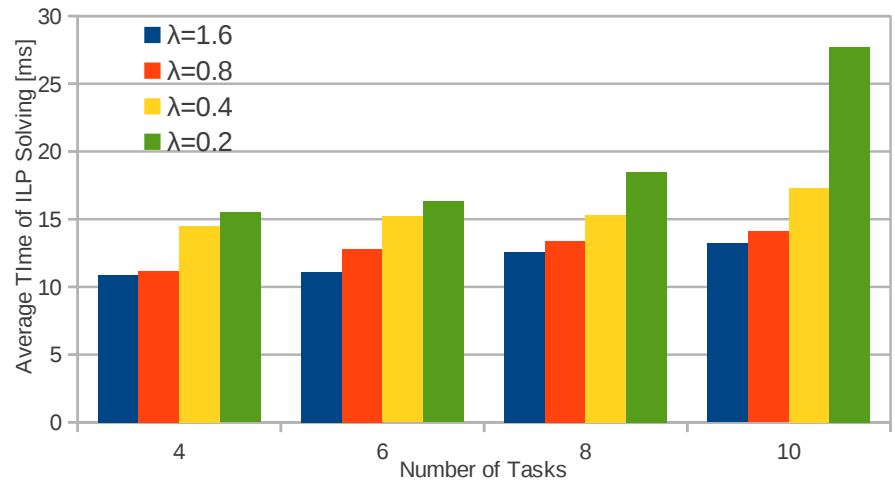

Figure 5.10: Average time of solving ILP 
as shown in the results, good result quality and fast optimization convergence can still be achieved using a larger $\lambda$.

The average time (in milliseconds) of solving the ILP problem formulated in Section 5.5.1.3 is illustrated in Figure 5.10. Note that the case of $\lambda=0$ is not presented, since all functional modes have their Pareto spaces stored in memory under this scenario. As can be seen, the overall average time that the CPLEX solver took is less than $15 \mathrm{~ms}$ among all the experimental settings, which is negligible comparing with the efforts of solving the first two steps in the off-line design phase.

\subsubsection{Run-Time}

The ultimate goal of the whole design optimization procedure is to make sure that, whenever the system changes modes or states, it always adapts to the situation with an efficient operation point. The design-time phase is our first step for achieving the goal. Our second set of experiments took a close look at the run-time system. To this end, we have considered the 400 cases discussed above, and we have executed the run-time system for each of them. For each of these 400 experiments, the run-time system has been exposed to a randomly generated sequence of 20 external requests. Each request is of the form $\left(M, Q o C^{R}, I D A^{R}, P o w^{R}\right)$ representing the mode and the constraints to be considered when choosing a new operation point. At least one of these four components of the new request differed from the previous request in the same sequence. For each case handled by the run-time system, we registered the QoS delivered with the selected operation point. Based on these experiments, we have answered the following three questions.

1) How is the QoS provided by our run-time algorithm compared to the situation when all Pareto spaces are stored in memory? This "ideal" situation means that, for all requests, the Pareto space corresponding to the requested mode is available (see Section 5.5.2.1) as opposed to having to extrapolate operation points from derived solution spaces in certain situations (see Section 5.5.2.2). In Figure 5.11 , we show the average degradation of QoS provided by the run- 


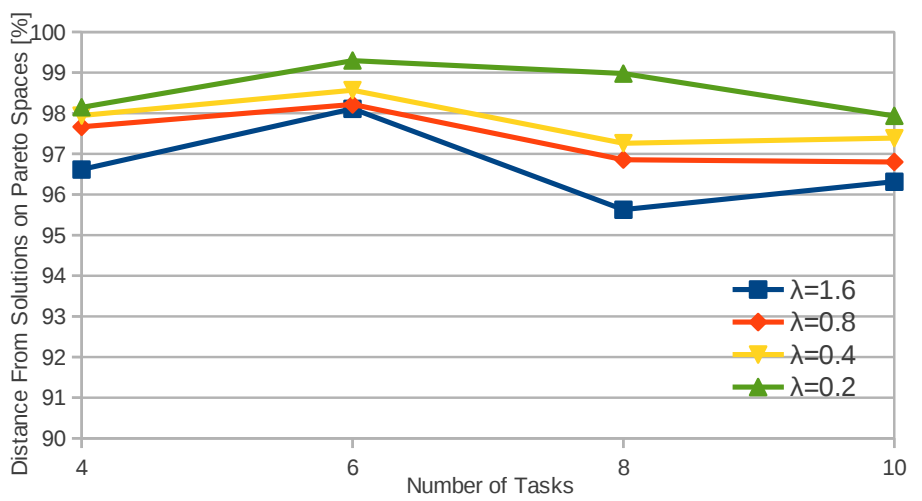

Figure 5.11: Average distance (\%) from solutions on Pareto spaces

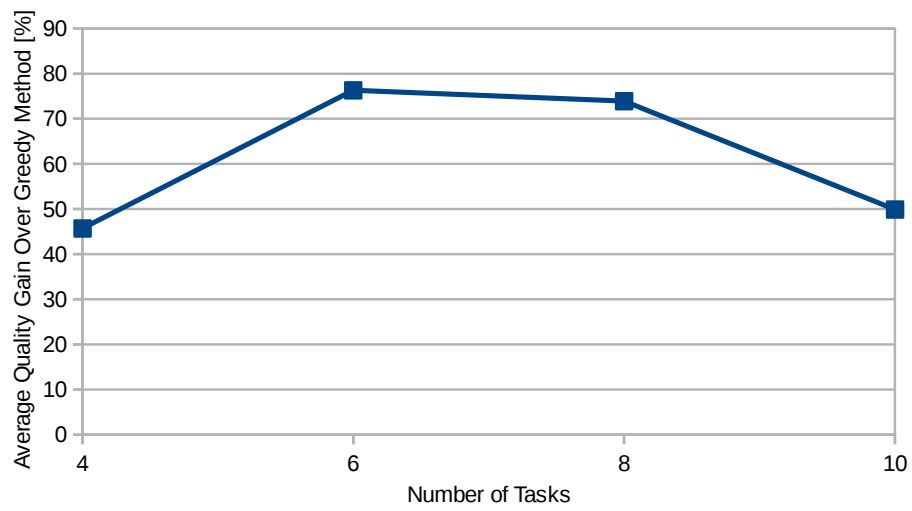

Figure 5.12: Average performance improvement over greedy method

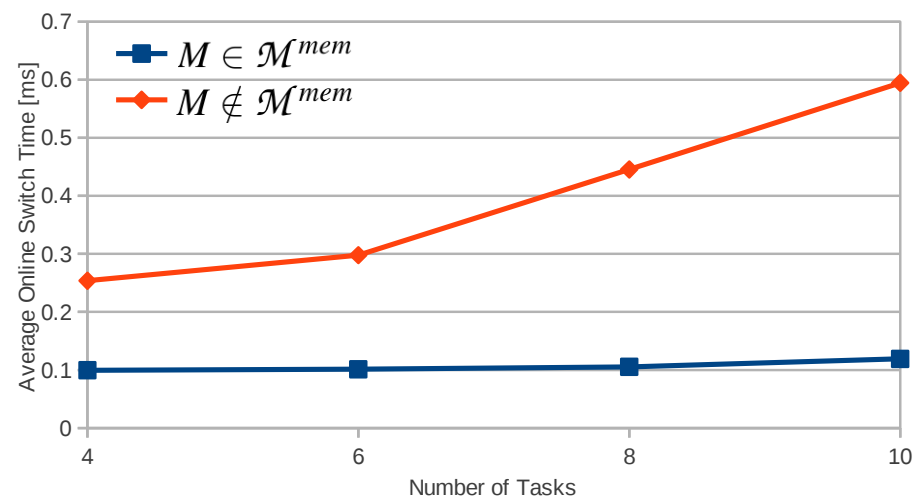

Figure 5.13: Average time of on-line operation point adaptation 
time system compared to the hypothetical case that Pareto spaces corresponding to all functional modes are available in memory. Not surprisingly, the degradations increase with the increase of $\lambda$, since the number of stored Pareto spaces decreases. For $\lambda=0.2$, the probability is high that, for the mode to be entered, the Pareto space is already stored in memory, or, at least, the space corresponding to a very close supermode is available. This probability is decreasing with larger values of $\lambda$. Thus, in all cases, a larger number of Pareto spaces are stored if a smaller $\lambda$ is chosen. It is, however, important to note that even for $\lambda=0.8$ this deviation is about $3 \%$ or less. This proves the capacity of our run-time heuristic to find efficient operation points even in the absence of the Pareto space corresponding to the entered mode.

2) Does it, at all, make sense to store Pareto spaces? If one carefully follows the run-time heuristic in Algorithm 5 and the discussion in Section 5.5.2.2, one can observe the sequence in Line 16-18 in which available resources are redistributed to satisfy constraints and improve QoS. In Algorithm 5, this redistribution is performed on the resources originally occupied by tasks $\mathcal{M}^{\prime} \backslash \mathcal{M}$. In fact, one can imagine a run-time heuristic that uses a similar technique to find an operation point. Instead of relying on pre-stored Pareto spaces, this technique would consider the whole processor bandwidth available, and use the above "redistribution" method to assign resources upon solving a new request. Such a scenario corresponds to the extreme case of our run-time system in Algorithm 5, in which $\mathcal{M}^{\text {mem }}=\emptyset$ (no Pareto space stored), and only Line 16-20 are executed. We have implemented such a simple run-time system, and compared the obtained QoS with that returned by our Pareto-space-based approach with $\lambda=0.4$. Figure 5.12 depicts the average QoS gains produced by our proposed technique, which lie between $40 \%$ and $80 \%$. This proves that using pre-calculated Pareto spaces is considerably increasing performance compared to a simple greedy resource distribution method.

3) What is the time overhead of adapting to an operation point onthe-fly? Since our proposed run-time system will be used on-line, the induced time overhead is another critical aspect to look at. Therefore, we also measured the execution times of the aforementioned run-time 
technique. Two situations need to be analyzed separately, that are when the Pareto space of the corresponding mode is available in memory, i.e., $M \in \mathcal{M}^{m e m}$ and when it is not, i.e., $M \notin \mathcal{M}^{m e m}$. The time overheads (in milliseconds) are illustrated in Figure 5.13. We can see that our run-time manager, on average, took roughly the same effort $(\approx 0.1 \mathrm{~ms})$ to find an operation point on all problem sizes under the case $M \in \mathcal{M}^{m e m}$. This can be correlated with the fact that, in the experiments, the number of operation points in the Pareto spaces returned by NSGA-II were set to be the same on all levels. Thus, the run-time system dealt with the same amount of information for selecting a point regardless of problem sizes. When the Pareto space for the corresponding mode is not available, i.e., $M \notin \mathcal{M}^{m e m}$, the average overheads for larger problem sizes were bigger than that for smaller sizes. This is due to that, for larger problem sizes, our run-time system operated on distributing resources to more tasks. However, as reflected by the upper line in Figure 5.13, the overhead grew linearly with problem sizes, since the computational load of the run-time manager in this case is proportional to the number of tasks.

\subsection{A Real-Life Case Study}

In order to further validate our proposed techniques, we applied the presented approach to a real-life benchmark that is a smartphone example. The system contains five applications, namely, a GSM codec [DB92], an MP3 decoder [Hag97], a JPEG codec [IJG98], an H.264 decoder [Cis14], and an NMEA 0183 parser [Moc14]. Each application is modeled as a task, and there is no data dependency among the tasks. They provide five different services to the user, i.e., phone calls/standby, online music playing, digital camera, video streaming, and navigation, respectively. The applications have been software profiled to retrieve the execution characteristics of the corresponding five tasks and task parameters. The hardware architecture was a DVFS-enabled SAMSUNG S3C2440 (ARM9) processor [SAM04].

We give a coexistence table as in Table 5.2, instead of presenting a full Hasse diagram to illustrate the functional modes. A "Y" (or "N") in Table 5.2 means that the two tasks can (or cannot) coexist at the 
Table 5.2: Task coexistence relations

\begin{tabular}{|c|c|c|c|c|c|}
\hline & GSM & MP3 & JPEG & H.264 & NMEA \\
\hline GSM & - & $\mathrm{Y}$ & $\mathrm{Y}$ & $\mathrm{Y}$ & $\mathrm{Y}$ \\
\hline MP3 & $\mathrm{Y}$ & - & $\mathrm{Y}$ & $\mathrm{N}$ & $\mathrm{Y}$ \\
\hline JPEG & $\mathrm{Y}$ & $\mathrm{Y}$ & - & $\mathrm{Y}$ & $\mathrm{Y}$ \\
\hline H.264 & $\mathrm{Y}$ & $\mathrm{N}$ & $\mathrm{Y}$ & - & $\mathrm{Y}$ \\
\hline NMEA & $\mathrm{Y}$ & $\mathrm{Y}$ & $\mathrm{Y}$ & $\mathrm{Y}$ & - \\
\hline
\end{tabular}

same time in the system. For example, the MP3 task and the H.264 task cannot coexist, due to competition for the audio playback hardware. Therefore, the modes that contain both tasks are functionally excluded. The task parameters of the five applications are given in Table 5.3. Note that, the GSM task has no optional execution part, and delivers constant QoS value at every activation. The other four tasks may carry out additional executions for achieving extra performance enhancement, e.g., better sound or image quality, with respect to the given reward functions shown in the last column of Table 5.3. The weights of $m_{11}, m_{21}, m_{41}$, and $m_{51}$ are considered equal, and their sizes are $64,512,2048$, and 32 bytes, respectively.

We have conducted the off-line optimization steps with five different improvement factors, i.e., $\lambda=0,0.2,0.4,0.8,1.6$. The memory limitation is set as $70 \%$ of total required memory size of the candidate Pareto spaces obtained from the Hasse diagram exploration ${ }^{3}$ with $\lambda$. The system has been exposed to a run-time test sequence consisting of 10 representative evaluation scenarios (first two columns of Table $5.4)$, each of which is of form $\left(M, Q o C^{R}, I D A^{R}, P o w^{R}\right)$ as seen in the previous section. The system runs under each scenario for a certain period between 5 seconds to 10 minutes which represents the most common use case of mobile phones. The ten consecutive scenarios are used to simulate different on-line requirements that the system may face. For example, the mode that the system is going to switch

\footnotetext{
${ }^{3}$ As an exception for $\lambda=0$, we remove the memory limitation to make sure that all modes have their Pareto spaces stored in memory for comparison purposes as in Section 5.6.2
} 


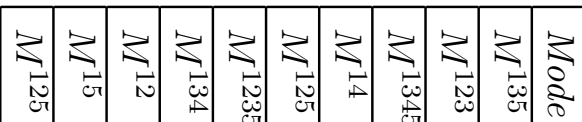

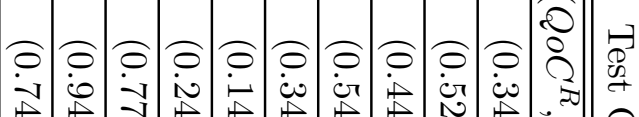

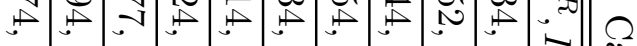

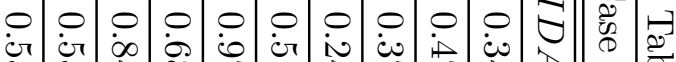

剀

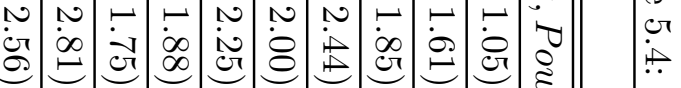

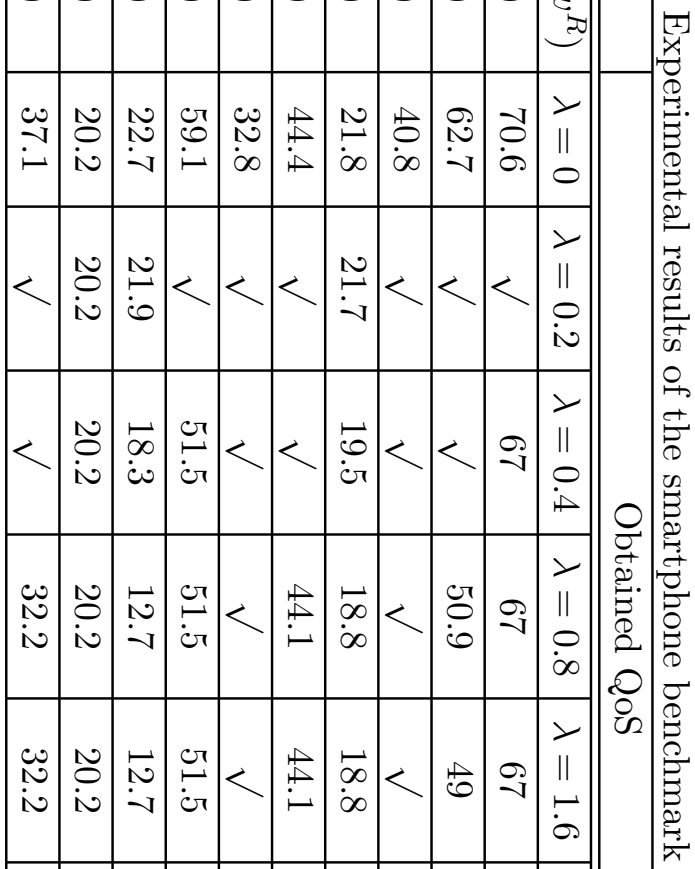

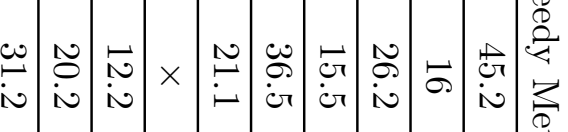

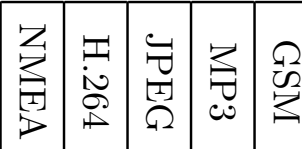

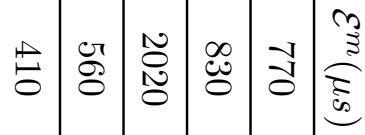

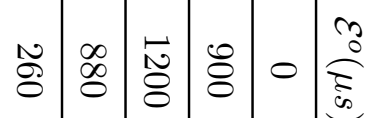

$\infty \tilde{D}^{\circ}$

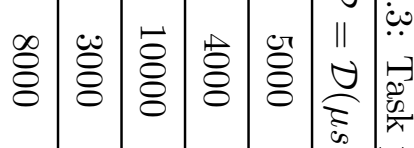
布

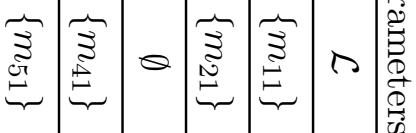

ט

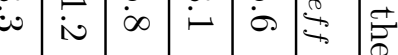

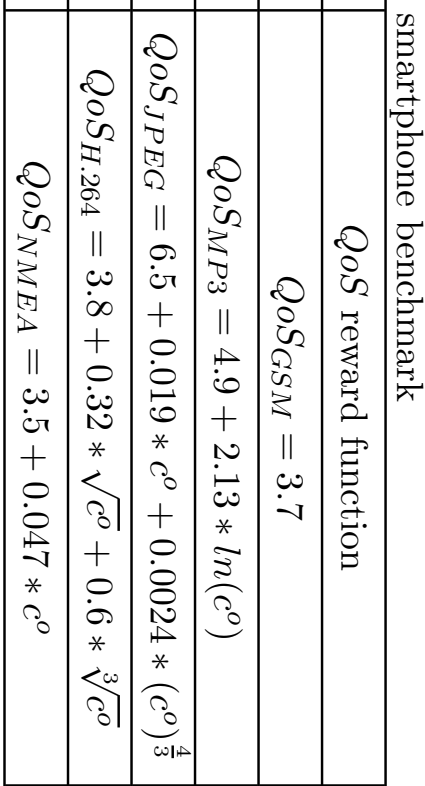


to can contain many tasks, e.g., $M^{1235}$ and $M^{1345}$, or only two tasks, e.g., $M^{12}$ and $M^{15}$. Additionally, some modes may appear more often than the others, e.g., $M^{125}$, for various reasons. At the same time, the concrete security and power constraints may vary, for example, one or both security requirements are high, e.g., (0.94, 0.55, 2.81); the power budget is very limited, e.g., $(0.34,0.34,1.05)$; or all the constraints are tight, e.g., $(0.77,0.84,1.75)$.

The QoS values that the system delivered under the experimental setups are presented in Table 5.4. The first two columns indicate the corresponding test case. The 3rd column depicts the results as if the Pareto space was stored in memory for that mode, and, thus, represents the best results. The 4 th to 7 th columns show the QoS values obtained using different $\lambda$ values in the off-line phase. For comparison purpose, we also conducted the greedy method described in Section 5.6.2 (in which the run-time optimization is directly applied with no Pareto space stored in memory) on the test sequence. The results from the greedy method are shown in the last column.

The check marks mean that the Pareto space for the mode is saved in memory. Therefore, the same result is retrieved as that in the third column (for $\lambda=0$, all Pareto spaces are stored in memory see footnote 3 ). The cross mark shows that the greedy method cannot find a solution for mode $M^{134}$ meeting all constraints $(0.24,0.68,1.88)$ simultaneously. As can be noticed in Table 5.4, for $\lambda=0.2$, Pareto spaces are available in 7 cases (in which case, the best results can be obtained); and high QoS values (very close to those in Column 3 of Table 5.4) can be reached in the other three cases. When choosing $\lambda=1.6$, the system can still produce much higher QoS than the greedy method. On average (discarding the 7th case in which the greedy method failed to find a satisfiable solution), our approach with $\lambda=1.6$ achieved $46.2 \%$ higher QoS than the greedy method.

The off-line optimization time and the on-line mode switching time are presented in Figure 5.14. The x-axis shows the five different settings of improvement factor $\lambda$. The optimization time of our off-line design phase for different $\lambda$ is represented by the continuous line, and is aligned to the $y$-axis on the left (in seconds). The time for finding an operation point at run-time using the proposed on-line techniques 


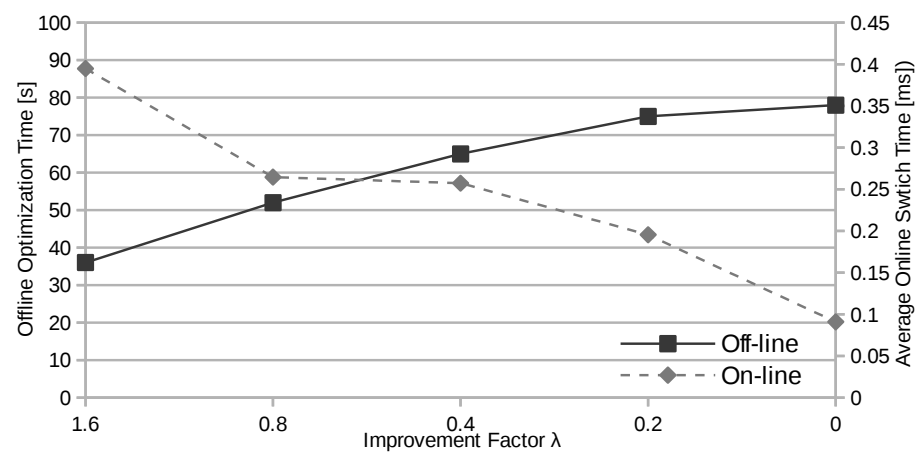

Figure 5.14: The off-line and on-line optimization overheads

is captured by the dashed line, and is aligned to the secondary y-axis on the right (in milliseconds). As can be noticed, the off-line optimization took longer time to finish for smaller $\lambda$, since more modes on the Hasse diagram got visited. Meanwhile, as $\lambda$ becomes smaller, the time overhead of the on-line phase is reduced. This is because that, with smaller $\lambda$, more Pareto spaces were stored in memory (since the memory limitation is set as $70 \%$ of the total required memory of $\mathcal{M}^{i m p l}$ ), and, consequently, more solutions could be directly obtained without deriving solution spaces and further processing the solutions. However, our proposed on-line heuristic is very efficient, and used only $0.24 \mathrm{~ms}$ to retrieve a solution on average $(0.72 \mathrm{~ms}$ in the longest case). Also worth mentioning, the time overhead of the on-line phase optimization was, in the worst case, $0.72 / 5000=0.014 \%$ of the duration of the next mode that is to be switched into. Therefore, the time overhead of our on-line heuristic is negligible.

\subsection{Summary}

In this chapter, we have presented a set of design optimization techniques for secure multi-mode embedded systems with dynamic task sets. We first analyzed a design problem for a single mode with four dimensions, namely, confidentiality protection, intrusion detection, power optimization, and QoS optimization. And then we discussed how to cope with the potentially large number of functional modes. 
After that, we studied the problem of on-line choosing the best operational point. However, the problems that we face impose huge computational complexity for solving exactly. Therefore, in order to master the complexity issues, we proposed a heuristic based two stage scheme as an alternative. The efficiency of the proposed techniques has been demonstrated by extensive experiments including a real-life case study. Note that the presented techniques are general enough to be also applied with other design requirements of multi-mode RTESs.

So far, we have discussed protecting the security aspects of RTESs by deploying extra security protection mechanisms, e.g., cryptography and intrusion detection. However, the actual implementations of such protection mechanisms may be under attacks, which weaken the overall protection strength. In the next chapter, we shall elaborate on preserving security of the underlying IBC implementations. 


\section{Chapter 6}

\section{Design of A Secure Scheduler Against Differential Power \\ Analysis Attacks}

$C^{\text {HAPTER } 4}$ and 5 have detailed our design techniques for secure $\bigcup$ RTESs. The goal was to efficiently carry out implementations of security protection mechanisms. However, as shown in Section 3.2.1.3, implementations of the security protection mechanisms on embedded platforms may be under severe threats. In this chapter, we shall present our solutions to resist such threats.

In order to achieve confidential communications, cryptographic algorithms, e.g., IBCs, need to be applied. However, the underlying cryptographic implementations leak side-channel information, e.g., power and sound, to the surrounding environment, which can be exploited by the attacker to retrieve secret information of the system. One of the most powerful and representative side-channel attacks is the differential power analysis attacks (DPAs). In this chapter, we shall discuss our solution against the DPAs. Theoretical guarantees and preliminary experimental results are presented to demonstrate the efficiency of the presented solution.

This chapter is organized as follows. Section 6.1 introduces the re- 


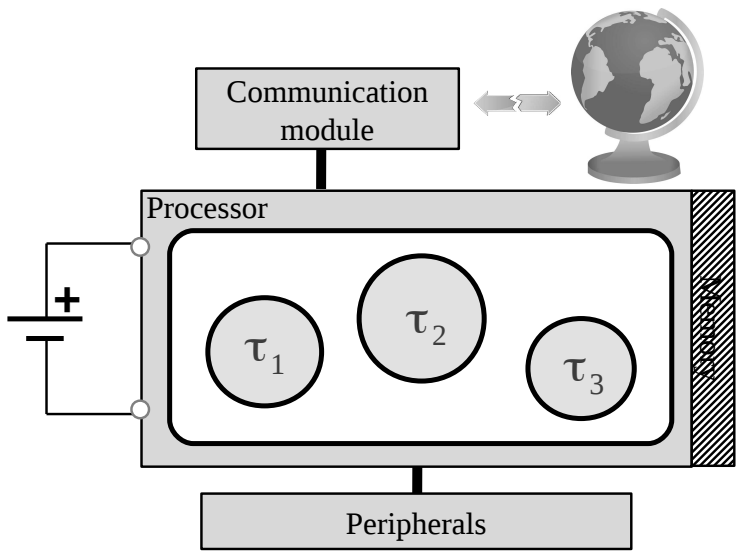

Figure 6.1: An illustrative system

lated hardware and application model. Section 6.2 and 6.3 present the fundamental motivations and an illustrative example, respectively. The presented concept is evaluated on two representative scheduling policies in Section 6.4. Section 6.5 elaborates our proposed scheduling policy that both guarantees the real-time properties of the system and resists DPA attacks. The results of the experimental evaluations on the proposed scheduler are presented in Section 6.6. Finally, Section 6.7 summarizes the chapter.

\subsection{System Model}

\subsubsection{Hardware Model}

The system we consider is a mono-processor RTES as shown in Figure 6.1. It is connected with various peripherals, e.g., sensors, actuators, and communication modules, via which it interacts with the environment or other peers. The microprocessor $\mu P$ and memory architecture are implemented with tamper-proof technology $\left[\mathrm{SCG}^{+} 03\right]$, making the computational operations and memory hierarchy not accessible. However, the power supply to the microprocessor may be approached. 


\subsubsection{Application Model}

There is a set of preemptive and periodic computation tasks $\mathcal{T}$ running on the microprocessor. The execution time and period (also the deadline) of task $\tau_{i} \in \mathcal{T}$ is denoted as $c_{i}$ and $\mathcal{P}_{i}$, respectively. Task $\tau_{i}$ may generate or/and receive a set of messages $\mathcal{L}_{i}$ to interact with other systems $\left(\mathcal{L}_{i}=\emptyset\right.$ if no message is associated). The length of message $m_{i j} \in \mathcal{L}_{i}$ is $l_{i j}$. The tasks are scheduled based on earliest deadline first (EDF), or rate-monotonic scheduling (RMS), or the proposed policy that will be elaborated in Section 6.5.

To make sure that the communication from/to the system is confidential, AES encryption and decryption operations [DR02] are required to be performed on all outgoing/incoming messages. The AES E/D procedure $\tau_{i j}^{A E S}$ on message $m_{i j}$ is considered to be part of the execution of task $\tau_{i}$ to simplify further discussions on scheduling. In other words, task $\tau_{i}$ (and execution time $c_{i}$ ) includes the normal computations of the task and the corresponding AES operations (and respective time overhead $c_{i j}^{A E S}$ ) on all associated messages $m_{i j} \in \mathcal{L}_{i}$.

\subsection{Time Dimension Shuffling Based Coun- termeasures}

As mentioned in Section 3.2.1.3, the measurement samples need to be noise-free and well aligned for DPA to be the most efficient. Having noise-free samples means that the power of the processor solely depends on the preformed operations and processed data. Thus, the attacker can make relatively accurate hypotheses on power consumptions, and the highest correlation $\rho_{\max }$ is obvious enough (see Section 3.2.1.3). This leads to the first type of countermeasures aiming at reducing the signal-to-noise-ratio (SNR) [TAV02], which is referred to as hiding in amplitude dimension. In this thesis, we shall focus on the second limitation of DPAs, that is, the less measured power values of the samples in column $P_{i}$ are due to executing the same leakage, the less efficient DPAs become.

As already discussed in this thesis, RTESs are demanded to provide strict guarantees on the timeliness of delivered results. Assigning 


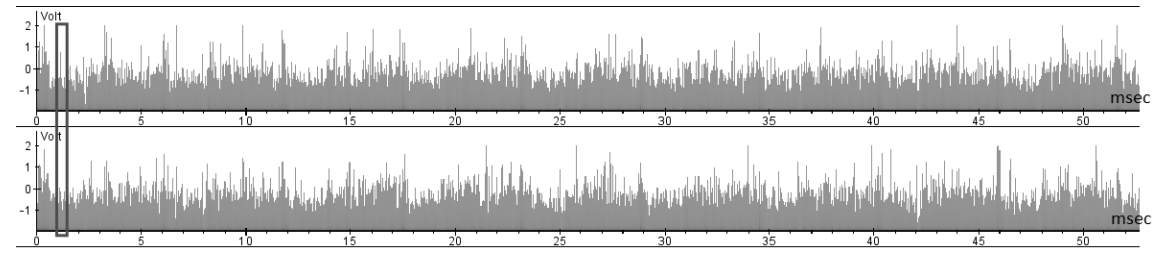

Figure 6.2: Power traces of AES on two messages with the same key

random delays into the execution as discussed in [CK09], to counteract against DPAs does not work in the context of RTES, since it may break the deadline constraints. However, dynamic scheduling algorithms [But11] serve, to a certain extent, the purpose of hiding in time dimension by their nature. Because of dynamic preemptions, the leakage fingerprints may occur at different times in different samples, which reduces the correlations between columns of the matrices $P$ and $H$ (see Section 3.2.1.3). The impact of real-time schedulers on DPA attacks has not been studied in the past. In this chapter, we propose an analytical framework to quantitatively capture the impact of task scheduling on the robustness of cipher implementations against DPA attacks, and also a scheduling policy that resists DPA attacks while guaranteeing real-time properties.

Figure 6.2 depicts two aligned power traces of AES on two different messages with the same key $K$. The leakage point of the first subkey $k_{1}$ occurs at $1.2 \mathrm{~ms}$ (highlighted in the rectangle). Assuming that two samples are sufficient to observe a high correlation with the hypothetical powers, then the attacker can already retrieve $k_{1}$, if the power traces like in Figure 6.2 are obtained. However, if the two leakages happened at different time, then she needs more samples to observe a dominating correlation for obtaining $k_{1}$. As just mentioned, DPA attacks work better if the operations at the same relative time point of all samples are the same. That is, in all the samples, the leakage points occur at the same relative time with respect to the starting time of the sample. Now the question is how to quantify the strength of an AES key under DPA attacks. 
Table 6.1: Task parameters for Figure 6.1

\begin{tabular}{|c|c|c|c|}
\hline Task & $c$ & $\mathcal{P}$ & $\mathcal{L}$ \\
\hline$\tau_{1}$ & 3 & 10 & $\emptyset$ \\
\hline$\tau_{2}$ & 8 & 20 & $\left\{m_{2}\right\}$ \\
\hline$\tau_{3}$ & 9 & 30 & $\emptyset$ \\
\hline
\end{tabular}

\subsection{Motivational Example}

As mentioned in Section 3.2.1.3, the highest probability $\hat{p}$ that the leakage point occurs at the same relative time point $\hat{t}$ determines the robustness of the key under analysis. One way to get $\hat{p}$ is through simulation of system execution, that is, to simulate and record the system execution and then to apply statistical methods on the recorded schedule. Thereafter, the robustness of the key can be calculated using Equation 3.10. Let us consider a simple example with three tasks as given in Table. 6.1. Task $\tau_{1}$ and $\tau_{3}$ do not have message communication, while, task $\tau_{2}$ generates one 128-bits long message $m_{2}$, and encrypts it with AES before sending it out over the communication module. In this example, we assume that the 8-th time unit of $\tau_{2}$ is the leakage point.

If the system is scheduled by the EDF policy, we shall get the schedule as shown in Figure 6.3 (a) for one hyperperiod $\mathcal{H P}$ of the task set $\mathcal{T}$. The hyperperiod of $\mathcal{T}$ is the least common multiplier of all task periods, i.e., $\mathcal{H P}=60$ in this case, and is the minimal time interval that the task execution pattern repeats. In other words, the system schedule is identically repeating after one hyperperiod. The gray rectangles represent the normal task executions including the non-leakage parts of AES, and the rectangles with hatching depict the leakage points. Assuming that the attacker measures the $\mu P$ power at each time unit, she will then obtain 60 discrete power values, each of which corresponds to a specific operation, e.g., task execution or AES, retrievable from the simulated schedule in Figure 6.3 (a). After that, she divides the whole obtained power trace into 3 individual samples based on $\mathcal{P}_{2}=20$ to align the samples (as depicted in Figure 


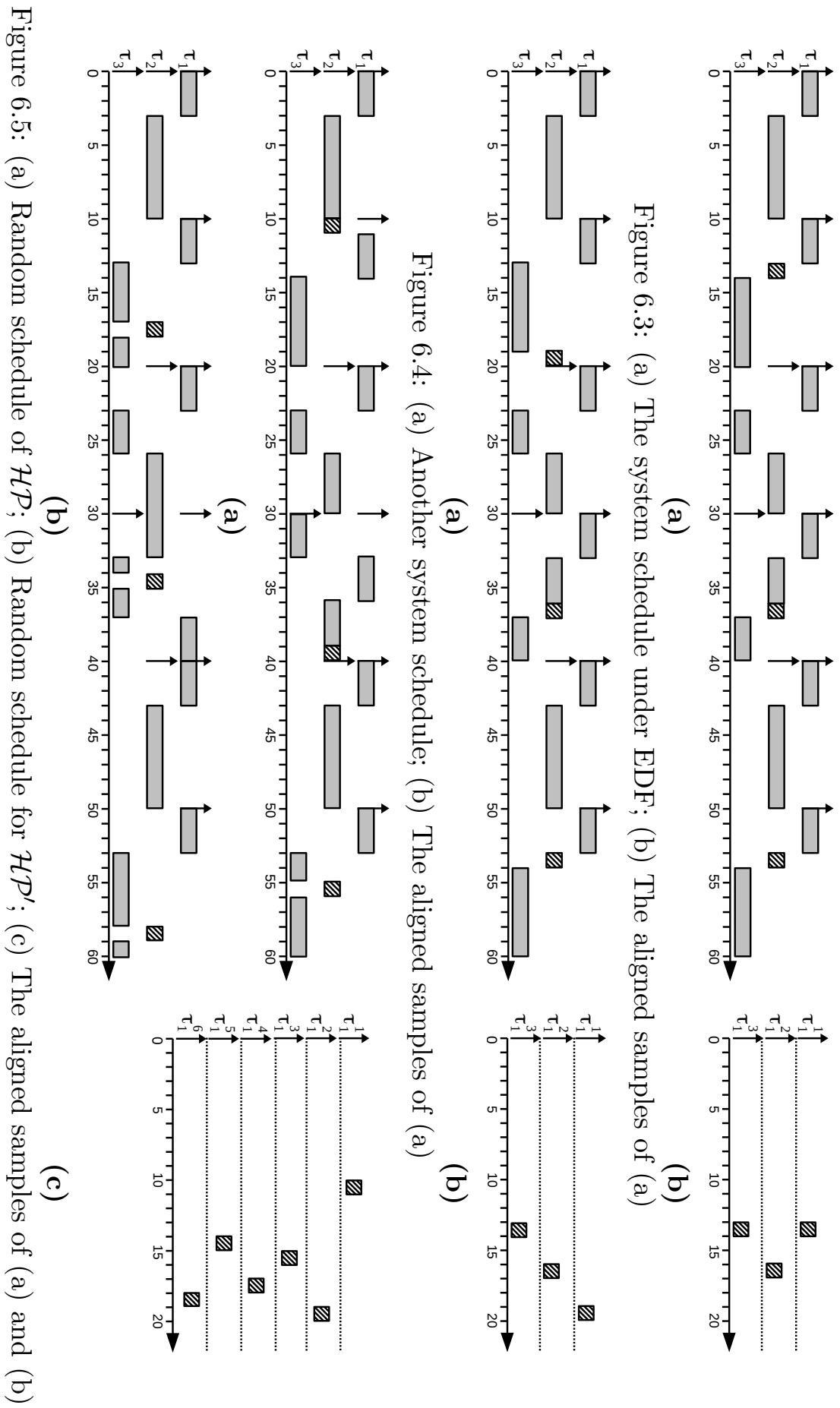


6.3 (b)). To highlight the leakage points, we do not show the nonleakage executions (the gray rectangles), which are independent from the leakages. After the hyperperiod, the same schedule will repeat. Therefore, we can find that the leakage occurs at relative time $\hat{t}=13$ with the highest probability $\hat{p}=\frac{2}{3}=0.67$. By now, we can calculate the robustness of the key $K_{2}$ using Equation 3.10, i.e., $\mathcal{R}_{2}=180$.

However, this does not seem to be a satisfying solution. An obvious alternative of scheduling the tasks is, for instance, to exchange the execution order of the first leakage point at $t=13$ with the first fraction of $\tau_{3}$ from $t=14$ to $t=19$ as shown in Figure 6.4 (a). If we align the samples as explained just now, we would get the result as in Figure 6.4 (b). We can observe that, now, the three leakage points occur at three different times, i.e., $t=20, t=16$, and $t=13$. However, this schedule repeats after one hyperperiod, making $\hat{p}=\frac{1}{3}=0.33$. Then the robustness of $K_{2}$ is $\mathcal{R}_{2}^{\prime}=620$.

An even better solution will be an enhanced random scheduler that can both guarantee the deadlines and reduce the value of $\hat{p}$. Figure 6.5 (a) illustrates the simulation of the system with such a scheduler under the first hyperperiod $[0, \mathcal{H P})$. As can be noticed, the leakage points occur at three different times, respectively. Furthermore, due to the randomness introduced by the scheduler, the simulation of the next hyperperiod (shown in Figure 6.5 (b)) gives a different schedule than Figure 6.5 (a). Thereby, if we align the two hyperperiods, we can get the samples as in Figure 6.5 (c). Now in this new scenario, in order to get the actual $\hat{p}$, we need to simulate the system execution for an extended timespan instead of just on hyperperiod, and, consequently, we would get $\hat{p}=0.11$ and $\mathcal{R}_{2}^{\prime \prime}=5460$.

\subsection{Proof of Concept on Existing Schedulers}

We have evaluated the impacts of scheduling policies against DPA attacks on two representative real-time schedulers, namely EDF and RMS [LL73]. We have conducted experimental analyses on a Linux machine with a quad-core Intel Xeon $2.66 \mathrm{GHz}$ CPU. The test applications were randomly generated under the requirements of different evaluation scenarios. Each task is associated with at most one mes- 
sage having length (in AES blocks) randomly selected from the set $\{0,1,2,3,4\}$ with probability $\{50 \%, 12.5 \%, 12.5 \%, 12.5 \%, 12.5 \%\}$, respectively. If $l_{i j}=0$, then $\tau_{i}$ does not have any communication demand, i.e., $\mathcal{L}_{i}=\emptyset$. The number of AES leakage fingerprints depends on the number of blocks of the message. We carried out experiments on EDF and RMS under three different scenarios to study the influence of the scheduler against DPA attacks from different perspectives.

\subsubsection{Evaluation under Different Processor Utilizations}

Now let us first have a look at how processor utilization influences the robustness of the system against DPA attacks under EDF and RMS. In order to judge which scheduler outperformed the other in an experiment, we have the following definition that focuses on which scheduler gives higher robustness for a key $k$ :

$$
f\left(\mathcal{R}^{E D F}(k), \mathcal{R}^{R M S}(k)\right)= \begin{cases}1 & , \text { if } \mathcal{R}^{E D F}(k)>\mathcal{R}^{R M S}(k) \\ 0 & , \text { if } \mathcal{R}^{E D F}(k)=\mathcal{R}^{R M S}(k) \\ -1 & , \text { if } \mathcal{R}^{E D F}(k)<\mathcal{R}^{R M S}(k) .\end{cases}
$$

Then, the overall performance indicator $\mathcal{F}$ for a set of experiments, i.e., the robustness of the secret key $\operatorname{Key}\left(\tau_{i j}^{A E S}\right)$ from all experiments under the same evaluation criteria, is defined as follows,

$$
\begin{aligned}
& \mathcal{F}\left(\operatorname{Key}\left(\tau_{i j}^{A E S}\right)\right)= \\
& \frac{1}{n} \sum_{\gamma=1}^{n} f\left(\mathcal{R}_{\gamma}^{E D F}\left(\operatorname{Key}\left(\tau_{i j}^{A E S}\right)\right), \mathcal{R}_{\gamma}^{R M S}\left(\operatorname{Key}\left(\tau_{i j}^{A E S}\right)\right)\right),
\end{aligned}
$$

where, $n$ is the total number of valid experiments where $\mathcal{L}_{i} \neq \emptyset$. The result $\mathcal{F}\left(\operatorname{Key}\left(\tau_{i j}^{A E S}\right)\right)$ captures the average overall superiority of the EDF scheduler over RMS. If EDF delivers higher robustness for $\operatorname{Key}\left(\tau_{i j}^{A E S}\right)$ in more cases, $\mathcal{F}>0$. Similarly, if RMS outperforms EDF on average, $\mathcal{F}<0$. EDF delivers better results in all the experiments, if $\mathcal{F}=1$. While, $\mathcal{F}=-1$ would indicate that RMS gives higher robustnesses in all cases.

The results presented in Figure 6.6 were obtained under 9 different utilization levels $U$ from 0.5 to 0.9 . On each level, 200 test applications with 5 tasks were randomly generated. The results demonstrate 


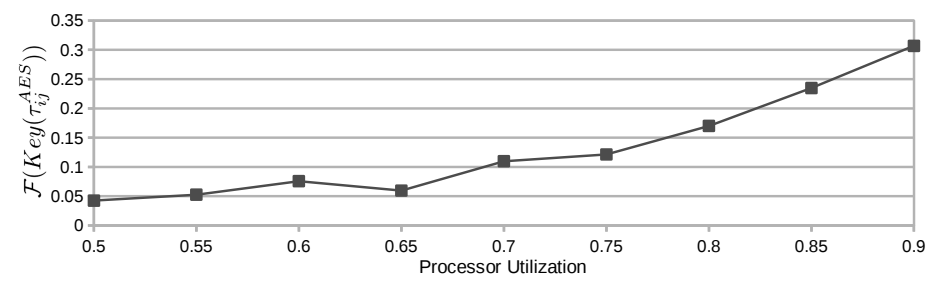

Figure 6.6: Results of 5 tasks under different processor utilizations

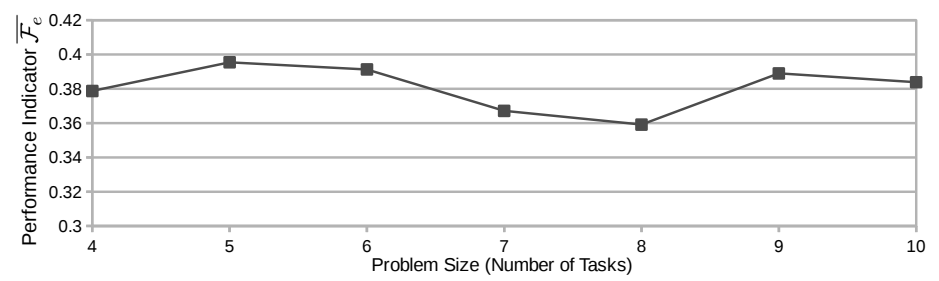

Figure 6.7: Results of different problem sizes

that, on average, EDF outperformed RMS in all situations. However, when the processor utilization was low, both policies provided similar protections on average, e.g., $\mathcal{F}=0.043$ at utilization $U=0.5$. This is because when the utilization is low, task executions are very sparsely spread over time. Therefore, the executions have little preemptions and, thus, occur more regularly at fixed time points for both EDF and RMS. As the utilization became higher, EDF delivered better and better robustness on average than RMS, e.g., $\mathcal{F}=0.307$ at utilization $U=0.9$. This can be explained by the fact that task priorities are dynamically changing based on their timelinesses in EDF, that introduces more randomness into the occurrences of tasks executions.

\subsubsection{Evaluation on Different Problem Sizes}

We also conducted experiments on seven different problem sizes having $|\mathcal{T}|=4,5, \ldots, 10$ tasks. On each problem size, we randomly generated 200 test applications with utilization $0.7 \leq U \leq 1$, and used the average performance indicator $\overline{\mathcal{F}_{e}}=\frac{1}{|\mathcal{T}|} \sum_{i=1}^{|\mathcal{T}|} \mathcal{F}\left(\operatorname{Key}\left(\tau_{i j}^{A E S}\right)\right)$ of all messages as the final indication for each problem size. The results are presented in Figure 6.7. From the results, we can notice that 


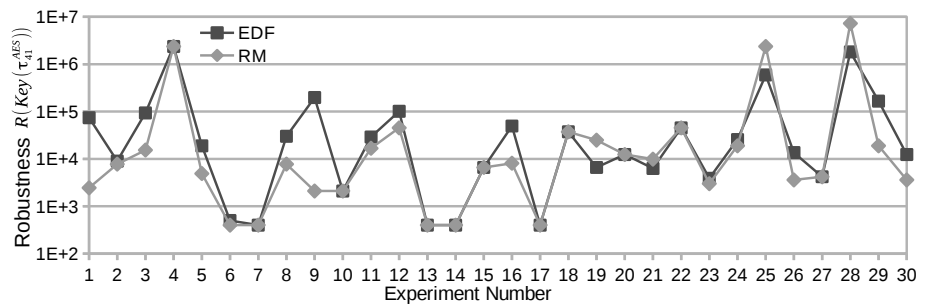

Figure 6.8: Robustness $R\left(\operatorname{Key}\left(\tau_{41}^{A E S}\right)\right)$ of 30 experiments under EDF and RMS

on average EDF outperformed RMS on all problem sizes under the utilization bound $0.7 \leq U \leq 1$.

\subsubsection{Evaluation on the Same Problem Size}

Figure 6.8 presents the actual robustness values of secret key $\operatorname{Key}\left(\tau_{41}^{A E S}\right)$ from 30 randomly selected valid experiments from the previous experiments, with number of tasks $|\mathcal{T}|=5$. As can be noticed, EDF provided better protection for $\operatorname{Key}\left(\tau_{41}^{A E S}\right)$ in 15 experiments, while RMS outperformed EDF in 4 tests (Experiment 19, 21, 25, and 28). In the rest of the cases, EDF and RMS gave the same robustness values. The experiments showed that, in the majority of cases, EDF produced higher robustness to $\operatorname{Key}\left(\tau_{41}^{A E S}\right)$. Also worth-mentioning, EDF delivered 4.83 times higher robustness on average in the experiments, and satisfied all deadline requirements. While, deadline misses were suffered in 2 cases when the system was scheduled with RMS. However, we cannot conclude that EDF was always preferable, since, in several cases, RMS delivered excellent results while also satisfying deadline constraints for all tasks, e.g., Experiment 19 and 28.

\subsection{A New Scheduling Policy for Thwarting DPA Attacks}

As demonstrated in the previous section, different scheduling policies have different influences on system robustness. However, neither EDF nor RMS was particularly designed to thwart DPA attacks. There- 
fore, in this section, we propose a new scheduling policy that aims to improve system robustness while satisfying all deadline constraints. For illustration purposes, we make the following simplification in the rest of this section: each task is associated with at most one message encryption occurring at the end of its execution, and the leakage point happens at the last $\delta$ time units of the task execution time. However, all the presented techniques and analyses can be easily extended to more practical cases in which a task may be associated with multiple messages, and the leakage point can occur anywhere within the task execution time.

\subsubsection{Proposed Scheduler: SPARTA}

What we propose in this section aims at the reduction of the alignment probability of leakage points in the columns of matrix $P$ (see Section 3.2.1.3). Such an approach of implementing countermeasures is often referred to as hiding in time dimension [MOP07]. Note that this type of countermeasure techniques are compatible with those implementing the hiding in amplitude dimension concept. It is recommended to implement multiple countermeasures to achieve the best protection.

In this section, we present our EDF-based scheduler named SPARTA and related theoretical guarantees. We use the following notations for describing the scheduler:

- $L_{s}$ : An ordered list of active tasks within time-interval $\left[T_{s}, T_{s+1}\right)$.

- $L_{s}^{\prime}$ : A list of tasks picked by SPARTA to be executed within $\left[T_{s}, T_{s+1}\right)$. Note that $L_{s}^{\prime} \subseteq L_{s}$.

- $r t_{i}$ : The execution time of a task $\tau_{i}$ to be carried out within a given time-interval.

- $e t_{i}$ : The execution time of a task $\tau_{i}$, ignoring the execution time of its leakage point, to be carried out within a given timeinterval; $e t_{i}=r t_{i}-\delta$

- $t_{i}^{\prime}$ : The time instant where the leakage of task $\tau_{i}$ starts.

- $\mathcal{X}_{i}$ : The leakage time-interval of task $\tau_{i}$, i.e., $\mathcal{X}_{i}=\left[t_{i}^{\prime}, t_{i}^{\prime}+\delta\right)$. 
- $Z_{s}$ : An ordered list of leakage points within $\left[T_{s}, T_{s+1}\right)$.

In the following, we describe the workflow of SPARTA:

1. The scheduler is activated at each task arrival. The time of the $s$-th activation of SPARTA is denoted as $T_{s}$. At time $T_{s}$, the scheduler considers the set of tasks to be executed within time-interval $\left[T_{s}, T_{s+1}\right)$, where $T_{s+1}$ is the time of the next task arrival. Then SPARTA decides a concrete schedule of these tasks to help thwart DPA attacks.

2. Unlike the EDF policy, which, at each task finish, executes the task that is closest to its deadline, SPARTA determines the schedule for the whole set of tasks that will be executed within $\left[T_{s}, T_{s+1}\right)$. It first sorts the tasks that are ready for execution at time $T_{s}$ based on the proximity to their respective deadlines. The resulting sorted list is captured by $L_{s}$ and the first entry of $L_{s}$ contains the task closest to its deadline.

3. The scheduler walks through $L_{s}$, starting from its first entry, and continues picking a task from $L_{s}$, until processor bandwidth within $\left[T_{s}, T_{s+1}\right)$ is fully utilized, or $L_{s}$ becomes empty. The list of tasks picked by the scheduler is kept in the list $L_{s}^{\prime}$ and $r t_{i}$ captures the execution time of $\tau_{i}$ to be carried out within $\left[T_{s}, T_{s+1}\right)$. Therefore, once the scheduler finishes walking through $L_{s}$, the following relationship must hold:

$$
\left(\sum_{\tau_{i} \in L_{s}^{\prime}} r t_{i}=T_{s+1}-T_{s}\right) \vee\left(L_{s}=\phi\right) .
$$

4. In this step, SPARTA decides a concrete schedule of tasks by first assigning leakage points at different time instants. Note that $e t_{i}$ captures the execution time of task $\tau_{i}$ to be carried out within $\left[T_{s}, T_{s+1}\right)$, ignoring the execution time of its leakage point. Therefore, et $t_{i}=r t_{i}-\delta$ (recall that $\delta$ is the execution time of a leakage point), for tasks with leakage points, and $e t_{i}=$ $r t_{i}$, otherwise. SPARTA randomly picks a task $\tau_{x_{1}}$, which has leakage point, from $L^{\prime}{ }_{s}$ and randomly allocates the leakage point 
at time $t_{x_{1}}^{\prime}$. In order to preserve the timing constraints, $t_{x_{1}}^{\prime}$ must be within the time interval $\left[T_{s}+e t_{x_{1}}, T_{s+1}\right)$. Once $t_{x_{1}}^{\prime}$ is assigned, SPARTA randomly selects another task $\tau_{x_{2}}$ having leakage point and randomly assigns its leakage point at time $t_{x_{2}}^{\prime}$, where $t_{x_{2}}^{\prime}$ satisfies the following condition:

$$
t_{x_{2}}^{\prime} \in\left\{\begin{array}{l}
{\left[T_{s}+r t_{x_{1}}+e t_{x_{2}}, T_{s+1}\right), \text { if } t_{x_{1}}^{\prime} \leq T_{s}+e t_{x_{1}}+r t_{x_{2}}} \\
{\left[T_{s}+e t_{x_{2}}, T_{s+1}\right) \backslash \mathcal{X}_{z_{1}}, \text { otherwise. }}
\end{array}\right.
$$

In general, SPARTA keeps an ordered list $Z_{s}$ for the allocated leakage points. $Z_{s}$ is ordered based on the time of occurrence of a leakage point and the first entry of $Z_{s}$ holds the leakage point occurring the earliest in time. Let us assume $n$ leakage points have been assigned (i.e. $\left|Z_{s}\right|=n$ ) and these leakage points are ordered in $Z_{s}$ as follows: $t_{z_{1}}^{\prime}<t_{z_{2}}^{\prime}<\ldots<t_{z_{n-1}}^{\prime}<t_{z_{n}}^{\prime}$. To assign the leakage point of a task $\tau_{x_{n+1}}$ (from $L_{s}^{\prime}$ ), we choose $t_{x_{n+1}}^{\prime}$, satisfying the following criteria:

$$
t_{x_{n+1}}^{\prime} \in\left\{\begin{array}{c}
{\left[T_{s}+\sum_{i=1}^{n} r t_{z_{i}}+e t_{x_{n+1}}, T_{s+1}\right)} \\
\quad \text { if } t_{z_{n}}^{\prime} \leq T_{s}+\sum_{i=1}^{n-1} r t_{z_{i}}+e t_{z_{n}}+r t_{x_{n+1}} \\
{\left[T_{s}+\sum_{i=1}^{n-1} r t_{z_{i}}+e t_{x_{n+1}}, T_{s+1}\right) \backslash \mathcal{X}_{z_{n}},} \\
\quad \text { if } t_{z_{n}}^{\prime}>T_{s}+\sum_{i=1}^{n-1} r t_{z_{i}}+e t_{z_{n}}+r t_{x_{n+1}} \\
\quad \wedge t_{z_{n-1}}^{\prime} \leq \sum_{i=1}^{n-2} r t_{z_{i}}+e t_{z_{n-1}}+r t_{x_{n+1}} \\
\ldots \quad \text { ot } \\
\quad \text { otherwise. }
\end{array}\right.
$$

This step ends when all the leakage points are allocated.

5. Once all the leakage points are assigned, we try to glue the 
task executions as tight as possible to reduce the number of preemptions. Let us assume that the list $Z_{s}$ is ordered as follows: $t_{z_{1}}^{\prime}<t_{z_{2}}^{\prime}<\ldots<t_{z_{l-1}}^{\prime}<t_{z_{l}}^{\prime}$, where $\left|Z_{s}\right|=l$. Our scheduler picks $\tau_{z_{1}}$ and assigns the remaining execution time $e t_{z_{1}}$ directly before $t_{z_{1}}^{\prime}$. Similarly, the remaining execution time of $\tau_{z_{2}}$ (i.e. et $\left.t_{z_{2}}\right)$ is alloted before $t_{z_{2}}^{\prime}$. Once the remaining execution time of all the tasks in $Z_{s}$ has been allocated, the tasks in $L^{\prime}{ }_{s} \backslash Z_{s}$ (tasks without leakage points), are considered. In particular, each such task is allocated by greedily occupying the first available slots.

As can be noticed, we consider each task to be associated with at most one message, and the leakage point always occurs at the end of the task execution. However, it is worthwhile to mention that the scheduler can be extended to support the situations where a task is associated with multiple messages, and the leakage point occurs at an arbitrary position in the execution. Both aspects can be solved by dividing the task into subtasks based on occurrences of leakage points. Subsequently, the assignments of leakage points can be carried out on these subtasks similar to Equation 6.5.

\subsubsection{Properties of SPARTA}

Now let us present the important theoretical properties of SPARTA that are related to schedulability and complexity.

\subsubsection{Schedulability Guarantee}

SPARTA satisfies the following crucial property.

Property 6.5.1. Let us assume that $Z_{s}$ holds $l$ entries after all leakage points have been assigned and the list $Z_{s}$ is ordered as follows: $t_{z_{1}}^{\prime}<t_{z_{2}}^{\prime}<\ldots<t_{z_{l-1}}^{\prime}<t_{z_{l}}^{\prime}$, where $\left|Z_{s}\right|=l$. Our scheduler guarantees that for all $i \in[1, l]$, the remaining execution time et $t_{z_{i}}$ of task $\tau_{z_{i}}$ is assigned before $t_{z_{i}}^{\prime}$.

Proof. To prove for the general case, we consider an arbitrary leakage point $t_{z_{n}}^{\prime}$. Let us distinguish between the following scenarios to show that the assignment of $t_{z_{n}}^{\prime}$ is safe, i.e., $\tau_{z_{n}}$ is able to finish its execution before $t_{z_{n}}^{\prime}$. 
- Case I: All the tasks $\tau_{z_{i}, \forall i \in\{1,2, \ldots, n-1\}}$ have been processed from list $L_{s}^{\prime}$ before task $\tau_{z_{n}}$ (cf. step 4 of SPARTA).

- Subcase A: $t_{z_{n}}^{\prime}>t_{z_{n-1}}^{\prime}$, because $t_{z_{n}}^{\prime}$ cannot be placed before any $t_{z_{i}}^{\prime}$, where $i \in[1, n-1]$. From Equation 6.5, we can observe that this scenario must happen for the first case, i.e., when the lower bound of $t_{z_{n}}^{\prime}$ is at least $T_{s}+\sum_{i=1}^{n-1} r t_{z_{i}}+e t_{z_{n}}$. This directly implies the fact that the non-leakage part of $\tau_{z_{n}}$ can finish before $t_{z_{n}}^{\prime}$. This is because of the assignment via Equation 6.5, as $t_{z_{n}}^{\prime} \geq$ $T_{s}+\sum_{i=1}^{n-1} r t_{z_{i}}+e t_{z_{n}}$.

- Subcase B: $t_{z_{n}}^{\prime}>t_{z_{n-1}}^{\prime}$, but $t_{z_{n}}^{\prime}$ could have been placed before some $t_{z_{i}}^{\prime}$, where $i \in[1, n-1]$, according to Equation 6.5. This means $t_{z_{n}}^{\prime}$ might, at least, be assigned before $t_{z_{n-1}}^{\prime}$, according to Equation 6.5. Based on the constraints starting from the second condition in Equation 6.5, we can observe that $t_{z_{n-1}}^{\prime}>T_{s}+\sum_{i=1}^{n-2} r t_{z_{i}}+e t_{z_{n-1}}+r t_{z_{n}}=T_{s}+$ $\sum_{i=1}^{n} r t_{z_{i}}-\delta$. From our hypothesis, we have $t_{z_{n}}^{\prime}>t_{z_{n-1}}^{\prime}$. Therefore, $t_{z_{n}}^{\prime}>t_{z_{n-1}}^{\prime}>T_{s}+\sum_{i=1}^{n} r t_{z_{i}}-\delta$. This also implies directly that the assignment of $t_{z_{n}}^{\prime}$ is safe.

- Case II: There is at least one task $\tau_{z_{i}}(i \in[1, n-1])$ that was processed from list $L_{s}^{\prime}$ after task $\tau_{z_{n}}$ ( $c f$. step 4 of SPARTA). Consider the last task, say $\tau_{z y}(y \in[1, n-1])$, with leakage point assigned within $\left[T_{s}, t_{z_{n}}^{\prime}\right)$. The reason why $t_{z_{y}}^{\prime}$ can be assigned before $t_{z_{n}}^{\prime}$ is that there is sufficient processor bandwidth left for $\tau_{z_{y}}$ before $t_{z_{n}}^{\prime}$. From the constraints of Equation 6.5, we know this is possible only if $t_{z_{n}}^{\prime}>T_{s}+\sum_{i \in\{1,2, \ldots, n-1\} \backslash\{y\}} r t_{z_{i}}+e t_{z_{n}}+$ $r t_{z_{y}}$. Therefore, $t_{z_{n}}^{\prime}>T_{s}+\sum_{i}^{n} r t_{z_{i}}-\delta$. This proves that the assignment of $t_{z_{n}}^{\prime}$ is safe.

Schedulability test From Property 6.5.1, we can state that SPARTA guarantees the assignments of leakage points to be safe. This means that, for the tasks with leakage points $Z_{s}$, the demanded executions will be finished upon the respectively allocated leakage points, which 
are before the corresponding tasks' deadlines. This is due to the fact that, within the interval $\left[T_{s}, T_{s+1}\right)$, there is no task release or deadline constraint under the assumption of task deadline equal to task period. For the tasks with no leakage $L_{s}^{\prime} \backslash Z_{s}$, the corresponding executions will be greedily allocated to the first available slots (see Section 6.5.1). This ensures that no slack will be left between task executions. Since the task set $L_{s}^{\prime}$ is determined based on EDF policy, the free processor bandwidth after assigning the tasks in $Z_{s}$ is sufficient to accommodate the tasks in $L_{s}^{\prime} \backslash Z_{s}$ using SPARTA.

Therefore, SPARTA guarantees to schedule all tasks from the list $L_{s}^{\prime}$, which is the same set of tasks (along with their respective execution time to be carried out within the time-interval $\left.\left[T_{s}, T_{s+1}\right)\right)$ picked by a classic EDF scheduler. Since the proof is not restricted to a particular choice of $\left[T_{s}, T_{s+1}\right)$, i.e., $s$ is arbitrary, we can conclude that the schedulability test for SPARTA is exactly the same as of EDF, that is,

$$
\sum_{\tau_{i} \in \mathcal{T}} \frac{c_{i}}{\mathcal{P}_{i}} \leq 1
$$

\subsubsection{Upper-bound of Context Switches}

Lemma 6.5.2. Let us use $l_{s}$ to denote the length of $Z_{s}$, which is the ordered list of leakage points assigned within $\left[T_{s}, T_{s+1}\right)$ as in Property 6.5.1. Then the number of context switches ${ }^{1} C S_{s}$ introduced by allocating the tasks with leakage points satisfies $l_{s} \leq C S_{s}<2 l_{s}$.

Proof. The proof below goes through the tasks in $Z_{s}$ in the same order as SPARTA allocates their non-leakage executions. Remind that, SPARTA allocates the non-leakage part of a task $\tau_{z_{i}} \in Z_{s}$ into the closest available slacks prior to $t_{z_{i}}^{\prime}$ (see Section 6.5.1). It is obvious that no slack is left between the starting and finishing time of $\tau_{z_{i}}$, which may also contain other task executions (implying context switches). Execution of $\tau_{z_{i}}$ introduces at least one context switch when it starts execution. Extra context switches from $\tau_{z_{i}}$ happen

\footnotetext{
${ }^{1}$ We refer to the start of a task execution or continuation of a task after being interrupted for some time as a context switch.
} 


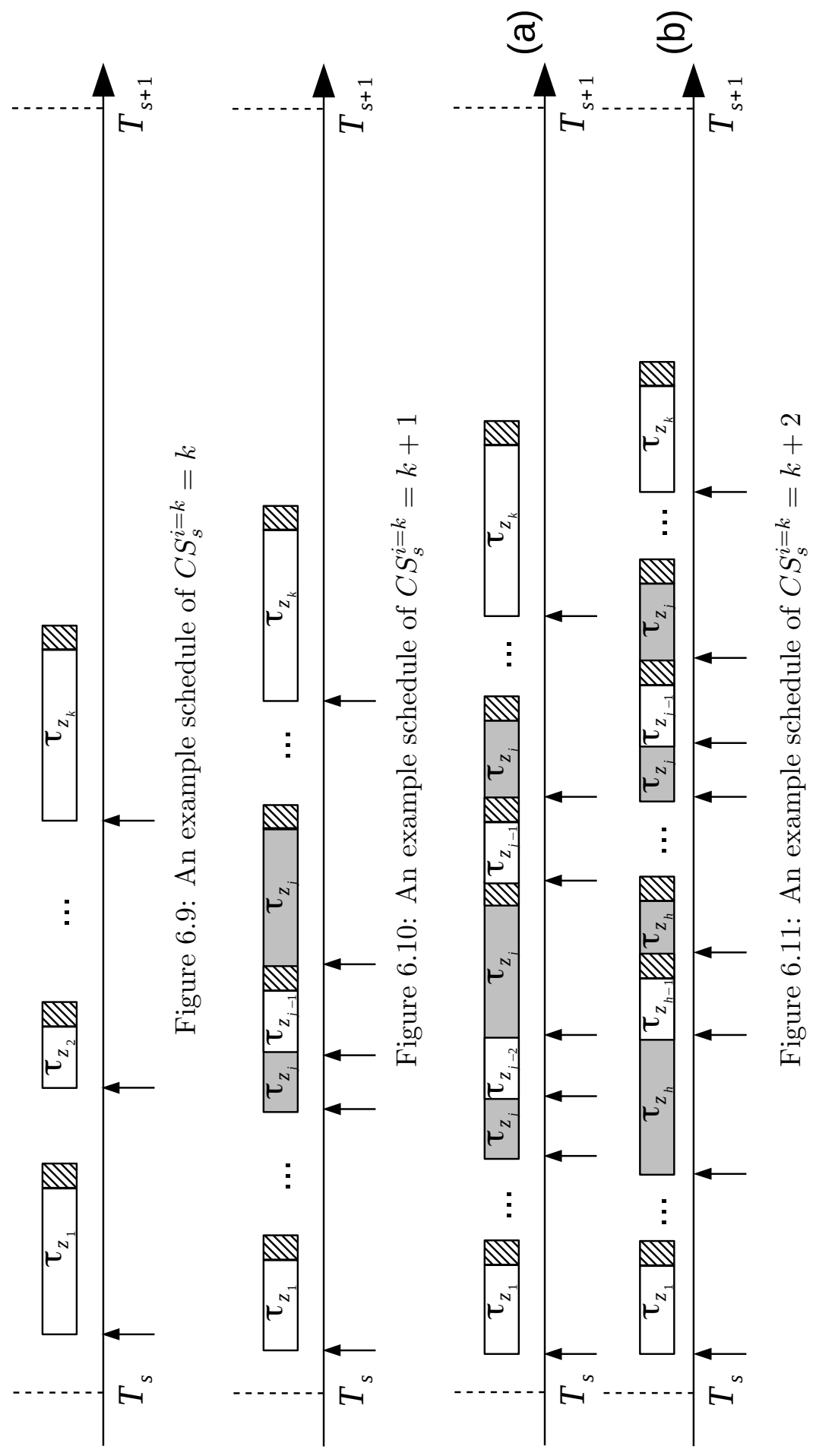


only when the execution of $\tau_{z_{i}}$ cannot fit within the first available slack, and has to expanded over multiple slacks. Now let us prove Lemma 6.5 .2 by induction.

- Base case: Let us look at the first task $\tau_{z_{1}} \in Z_{s}$, which is the first task to be allocated in the fifth step of SPARTA. The non-leakage part is allocated directly before the leakage, leading to $C S_{s}^{i=1}=1$. Thereby, Lemma 6.5.2 holds for $i=1$, as $1 \leq$ $C S_{s}^{i=1}<2$.

- Inductive step: Let us assume that, for $i=k$, Lemma 6.5.2 holds, that is, $k \leq C S_{s}^{i=k}<2 k$. The minimal number of context switches after allocating $\tau_{z_{k}}$ is $k$. Now we shall look at task $\tau_{z_{k+1}}$. The minimal number of context switches it may introduce is 1 as mentioned. Thereby, the left hand side of Lemma 6.5.2 holds $\left(k+1 \leq C S_{s}^{k+1}\right)$ for $i=k+1$. Then we focus on proving the right hand side of Lemma 6.5.2, i.e., $C S_{s}^{i=k+1}<2(k+1)$. From the assumption $k \leq C S_{s}^{i=k}<2 k$, we can find that the actual number of context switches until $\tau_{z_{k}}$ can only be one of the following cases:

- $C S_{s}^{i=k}=k$ : Since executing a task will at least introduce 1 context switch, no execution of $\tau_{z_{j}}: j \in[1, k]$ is spread over multiple slacks as shown in Figure 6.9 (the arrows indicate the context switches). In this case, the execution of $\tau_{z_{k+1}}$ can maximally expand over all the $k+1$ slacks. So the number of context switches introduced by $\tau_{z_{k+1}}$ is at most $k+1$. Therefore, $C S_{s}^{i=k+1} \leq k+k+1<2(k+1)$.

- $C S_{s}^{i=k}=k+1$ : In this case, there is only one task $\tau_{z_{j}}$ having its execution expanded over its previous task $\tau_{z_{j-1}}$ as shown in Figure 6.10, and there is no slack left between the two execution fractions of $\tau_{z_{j}}$. So the total number of available slacks is $k$. Consequently, the maximal number of context switches $\tau_{z_{k+1}}$ may introduce is also $k$. Therefore, $C S_{s}^{i=k+1} \leq k+1+k<2(k+1)$.

- $C S_{s}^{i=k}=k+2$ : In this case, there can be one task $\tau_{z_{j}}$ with execution expanded over two of its previous tasks as shown 
in Figure 6.11(a) or two tasks $\tau_{z_{h}}$ and $\tau_{z_{j}}$ with executions expanded over one of their respectively previous task as in Figure 6.11(b). Under both scenarios, the number of slacks left is $k-1$. So the maximal number of context switches that $\tau_{z_{k+1}}$ may introduce is also $k-1$. Therefore, $C S_{s}^{i=k+1} \leq k+2+k-1<2(k+1)$.

- $C S_{s}^{i=k}=2 k-1$ : This case can be caused by various scenarios, e.g., $\tau_{z_{k}}$ has its execution expanded over all the previous tasks or each task execution is split into two parts. However, under all possibilities, the total number of available slacks left is 2, that are before the starting of $\tau_{z_{1}}$ and after the end of $\tau_{z_{k}}$, respectively. So the maximal number of context switches that $\tau_{z_{k+1}}$ may introduce is 2 . Therefore, $C S_{s}^{i=k+1} \leq 2 k-1+2<2(k+1)$.

Thereby, we can conclude that Lemma 6.5.2 holds.

Property 6.5.3. The total number of system context switches introduced by SPARTA is at most 2 times of that implied by EDF.

Proof. Assuming that the number of tasks to be executed within $\left[T_{s}, T_{s+1}\right)$ is $n_{s}$, then the system has $n_{s}$ context switches if scheduled by EDF.

- $l_{s}=n_{s}$ : In this case, all tasks in $L_{s}^{\prime}$ have leakage points. As shown by Lemma 6.5.2 $\left(C S_{s}<2 n_{s}\right)$, Property 6.5.3 holds within $\left[T_{s}, T_{s+1}\right)$.

- $l_{s}<n_{s}$ : In this case, some tasks in $L_{s}^{\prime}$ have no leakage point. After allocating all tasks in $Z_{s}$, the actual number of context switches $C S_{s}^{i=l}$ is in the range $\left[l_{s}, 2 l_{s}\right)$. Similar to the proof of Lemma 6.5.2, we can prove the property by enumerating all the possible scenarios of $C S_{s}^{i=l}$. For the sake of conciseness, we only show the proof for the last case, i.e., $C S_{s}^{i=l}=2 l_{s}-1$, as follows.

In this case, the number of available slacks is 2 (as the case $C S_{s}^{i=k}=2 k-1$ in the proof of Lemma 6.5.2), and no slack exists 
within the allocated executions. SPARTA greedily assigns the tasks in $\tau \in L_{s}^{\prime} \backslash Z_{s}$ into the first available slacks starting from $T_{s}$. Note that there can be maximally only 1 task $\tau_{x}$ introducing 2 context switches, because the execution of $\tau_{x}$ needs to continue in the second slack only when the first slack is full. All the other tasks in $L_{s}^{\prime} \backslash Z_{s}$ can finish within either slack, yielding 1 context switch. Therefore, the maximal number of context switches from the tasks without leakage is $n_{s}-l_{s}+1$. So we have $C S_{s}=2 l_{s}-1+n_{s}-l_{s}+1=n_{s}+l_{s}<2 n_{s}$. So Property 6.5.3 holds within $\left[T_{s}, T_{s+1}\right)$.

Since the selection of $s$ is arbitrary, we can conclude that Property 6.5.3 holds in general.

\subsubsection{Complexity of SPARTA}

Property 6.5.4. The complexity of each SPARTA activation is $O\left(n^{2}\right)$, where $n$ is the total number of tasks in the system.

Proof. Let us focus on the SPARTA activation at $T_{s}$, and study the individual overhead of each step. In step 1 , it calculates $T_{s+1}$ by going through all $n$ tasks, thus, the complexity of step 1 is $n=O(n)$. In step 2 , SPARTA sorts the $n_{a}\left(n_{a} \leq n\right)$ ready tasks into a list $L_{s}$, leading to complexity $n_{a} l g\left(n_{a}\right)=O(n l g(n))$. In step 3, SPARTA selects the executions to be allocated from the sorted list $L_{s}$ until the interval $\left[T_{s}, T_{s+1}\right)$ is full, therefore, the complexity of step 3 is $n_{s}=O(n)$, where $n_{s}$ is the length of $L_{s}^{\prime}$. In step 4, SPARTA assigns leakage points one by one for all tasks with leakages. Before allocating a leakage point, SPARTA examines all the allocated leakages to decide a proper range. If the number of tasks with leakage is $l_{s}\left(l_{s} \leq n\right)$, then we can find that the complexity of step 4 is $0+1+2+\ldots+l-1=$ $\frac{1}{2}\left(l_{s}^{2}-l_{s}\right)=O\left(n^{2}\right)$. In the last step, SPARTA allocates the non-leakage executions greedily to the first possible slacks, yielding complexity of $n_{s}=O(n)$. Finally, we can find that the overall complexity of SPARTA is $O\left(n^{2}\right)$. 


\subsection{Experimental Evaluation}

In order to evaluate the achieved protection strength against DPA attacks enhanced by SPARTA, we have performed experimental evaluations under two representative criteria, i.e., different processor utilization levels and different problem sizes (different amount of tasks). Each task $\tau_{i}$ is associated with at most one message $m_{i}$ with length $l_{i}=1$. The task periods are chosen uniformly at random from $\{200,400, \ldots, 2000\}$ to avoid simulation of long hyperperiods. On each problem size, the task execution times were randomly generated under the current utilization level.

We have implemented SPARTA in C, and conducted experiments under a simulation environment on a Linux machine with a quadcore Intel Xeon processor and 8GB of memory. For illustration purposes, we compared the protection strength delivered by SPARTA with protection provided by EDF. We define the robustness of a set of experiments as the average achieved robustness over all keys, i.e.,

$$
\overline{\mathcal{R}}=\frac{1}{n} \sum_{i=1}^{n} \frac{1}{\left|\mathcal{L}_{i}\right|} \sum_{m_{j} \in \mathcal{L}_{i}} \mathcal{R}_{j},
$$

where $n$ is the number of experiments, and $\mathcal{L}_{i}$ is the set of messages in the i-th experiment. $\mathcal{R}_{j}$ is calculated according to Equation 3.10. The improvement achieved by SPARTA over EDF is defined by:

$$
\overline{\mathcal{I}}=\frac{1}{n} \sum_{i=1}^{n} \frac{1}{\left|\mathcal{L}_{i}\right|} \sum_{m_{j} \in \mathcal{L}_{i}} \frac{\mathcal{R}_{j}^{S P A R T A}-\mathcal{R}_{j}^{E D F}}{\mathcal{R}_{j}^{E D F}} .
$$

The two evaluation scenarios are presented as follows.

\subsubsection{Different Processor Utilizations}

In this set of experiments, we would like to study the influence of different processor utilizations on achieved protection strength. We conducted experiments with different processor utilization levels on the same problem size. That is, all experiments are with 6 tasks. The studied processor utilization levels are $U \in\{1, \ldots, 0.5,0.4\}$, and 


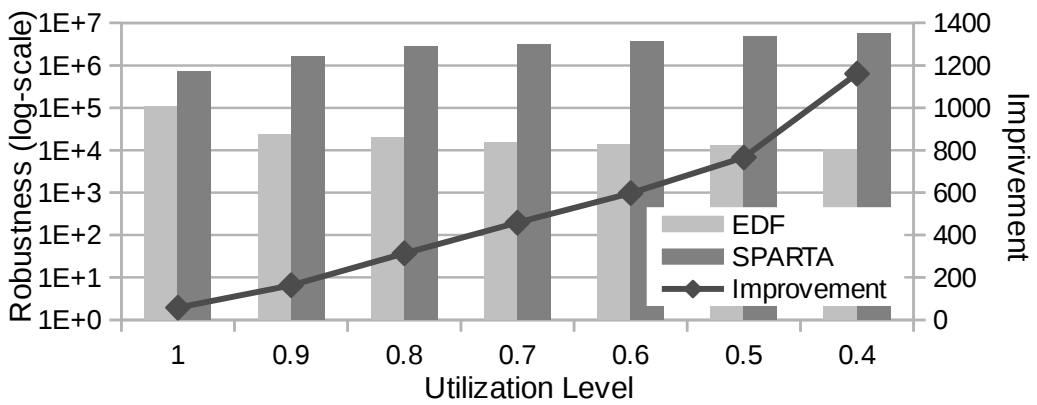

Figure 6.12: Results of SPARTA on different processor utilizations

on each level, 200 random test applications were generated with different task periods and execution times. The results are presented in Figure 6.12. The light gray and dark gray bars show the average robustness $\overline{\mathcal{R}}$ of EDF and SPARTA, respectively, with the corresponding setups, and are aligned to the primary (logarithmic) y-axis on the left. The curve indicates the improvement $\overline{\mathcal{I}}$ of each set of experiments, and is aligned to the secondary y-axis on the right. We can observe that the achieved improvements are gradually increasing as processor utilization drops (indicated by the line). We can also notice that SPARTA dominates EDF on all utilization levels, and it works better, when the processor is less loaded, due to the larger amount of free time slacks that it can make use of. Therefore, SPARTA has more flexibility in allocating leakage points when $U$ is low.

\subsubsection{Different Problem Sizes}

We now evaluate the performance of SPARTA on different problem sizes, i.e., systems with different amount of tasks. There are in total five sizes studied, namely, $|\mathcal{T}| \in\{4,6, \ldots, 12\}$. On each size, we randomly generated 200 test applications (having different task periods and execution times) with utilization $U=0.7$. The obtained results are shown in Figure 6.13, and the same representations are shared with those of Figure 6.12. We can find that SPARTA delivers nearly a constant improvement level (indicated by the line) compared with EDF, and the absolute delivered protection is higher on larger problem sizes. It is also worth mentioning that, for individual cases, 


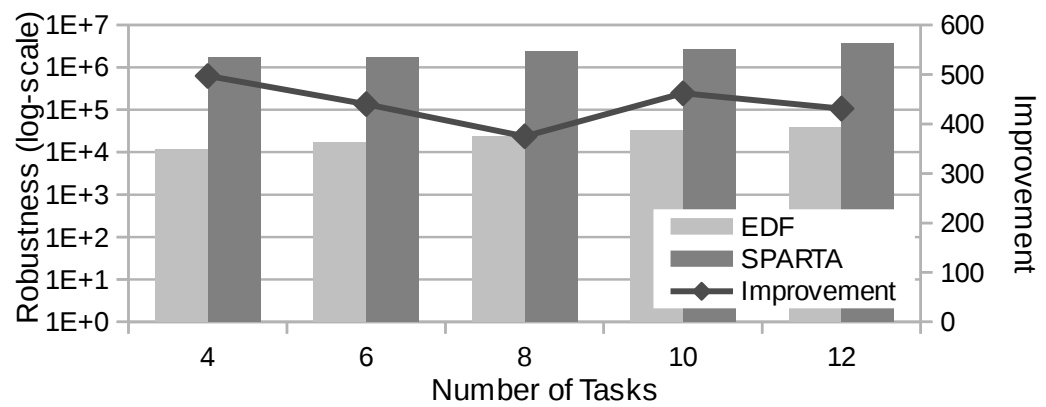

Figure 6.13: Results of SPARTA on different problem sizes

SPARTA delivered better protections for the AES secret keys in all the instances of experiments, and the average relative improvements is $\overline{\mathcal{I}}=441$.

\subsection{Summary}

Cryptographic algorithms, e.g., AES, have been deployed for protecting sensitive information. However, AES implementations in embedded platforms are known to be vulnerable towards side-channel attacks, e.g., DPA attacks. Therefore, the problem of how to design secure RTESs with robust AES must be carefully studied.

In this chapter, we evaluated the impact of the scheduling policy on the robustness of secret keys against DPA attacks using EDF and RMS, and showed that different scheduling policies do have different impacts on key robustness. Therefore, the designer must carefully choose the most suitable scheduler for the given application considering both deadlines and robustnesses.

After that, we have presented a new real-time scheduling policy SPARTA that thwarts DPA attacks. SPARTA shares the same schedulability test with EDF, and, at the same time, enhances security of the underlying AES implementations. We have conducted experiments to demonstrate that the protection strength of SPARTA is much better than that of EDF under different evaluation criteria. Finally, it is worth mentioning that, while in this chapter, we considered DPA attacks, similar analyses can be done for other side-channel 
CHAPTER 6. DESIGN OF A SECURE SCHEDULER AGAINST 134 DIFFERENTIAL POWER ANALYSIS ATTACKS

attacks based on the observations of other parameters, such as temperature and sound. 


\section{Chapter 7}

\section{Conclusions and Future Work}

$\mathrm{T}^{\mathrm{N}}$ this chapter, we shall summarize the main contributions of this 1 thesis (Section 7.1), and outline the potential directions of future work (Section 7.2).

\subsection{Conclusions}

In recent years, real-time embedded systems have been extensively used in critical application areas and also been unprecedentedly exposed to dynamic and open environments. Hence, the potential threats, as well as successful attacks, aiming at system security violations have drastically increased lately. Therefore, how to protect such systems is rapidly emerging to a critical problem. Meanwhile, RTESs are becoming much more complex and demanded to undertake multiple functionalities. How to achieve the existing requirements of a RTES design and, at the same time, guarantee the system security under potential threats has become a really difficult problem.

This thesis approaches the aforementioned design problems under three concrete design scenarios of RTESs, that are: 1. design and optimization techniques for communication confidentiality on distributed platforms (Chapter 4); 2. a global framework for secure multi-mode RTESs (Chapter 5); 3. a scheduling policy for resisting side-channel 
attacks (Chapter 6).

\subsubsection{Confidentiality-Aware Design Techniques}

In Chapter 4, we presented two confidentiality-aware design and optimization techniques for distributed RTESs, in which applications are modeled as task graphs. In the first case, we aim at achieving the best system affordable confidentiality protection for the message transmissions over the internal communication infrastructure under strict real-time requirements. In the second case, we consider more powerful platforms with FPGA accelerations, and focus on finding the minimal overall hardware overhead needed for implementing the designated cryptographic operations on the internal communication while achieving imposed constraints. We have carried out large amounts of experiments on both techniques, and also compared the obtained solutions with the optimums returned by solving corresponding CLP formulations.

\subsubsection{Secure Multi-Mode RTES Design Framework}

In Chapter 5, we addressed the design optimization problem of secure multi-mode RTESs. We considered different combinations of functionalities as modes, represented by the set of active tasks. Due to the potentially large number of tasks that may appear in the system, the amount of possible modes that can occur during system execution can be infeasible to explore. Moreover, even designing for one single mode is not a simple job, since the designer may want to obtain good performance along several conflicting objectives. Therefore, we presented a two stage heuristic approach to solve the problem efficiently. In the off-line stage, a set of Pareto-optimal solutions are developed and stored in memory. In the on-line stage, the system quickly adapts to concrete execution requirements making use of the solutions prestored in the off-line stage. The efficiency of the presented framework has been evaluated in extensive experiments, including a case study of a smartphone application. 


\subsubsection{Scheduling Against Side-Channel Attacks}

In Chapter 6, we outlined a scheduling policy, SPARTA, that actively thwarts side-channel attacks, more specifically, the differential power analysis attacks (DPAs). We observed that the power measurements corresponding to the leakage points need to be relatively noise-free and well aligned for DPA to be more efficient, and realized that dynamic schedulers introduce randomness into task schedules, thus, reducing the predictability of the executions. Consequently, the system is more robust towards potential DPAs. We verified the concept by evaluating the influence of EDF and RMS on difficulties of mounting DPAs under different scenarios. After that, we presented the SPARTA scheduler and its fundamental properties. Two different sets of experiments have been conducted on SPARTA to demonstrate its improved protection strength.

\subsection{Future Work}

This thesis has motivated the needs and addressed several concrete problems of designing secure RTESs. However, potential threats to modern RTESs exist in various aspects, and cannot be all covered by this thesis. Therefore, we would like to point out several possible directions for future research.

Among the important concepts of security, we have been focusing on techniques for delivering confidentiality to the internal communication of distributed RTESs. However, other aspects, e.g., integrity, can also be important in certain distributed RTESs. So an overall design framework that guarantees both confidentiality and integrity of system communication, while achieving timeliness, is needed.

For the multi-mode systems, we have depicted a holistic design approach that both considers the modes the system may run in and the constraints the system must satisfy simultaneously. However, we did not consider the probability of mode occurrences in the off-line phase. That is, all functional modes are assumed to occur in the system with the same probability, which may not capture the reality well. Thus, a framework that takes the variation of mode appearances 
into consideration can be an interesting extension to Chapter 5 .

Side-channel attacks are posing serious threats to the underlying implementations of cryptographic algorithms. So side-channel attack resistance is an indispensable aspect that must be ensured in the design of RTESs. Due to the actual timing and resource constraints of RTESs, countermeasures against side-channel attacks must be carefully studied. An interesting extension to SPARTA is to enhance system robustness for the RTESs in which task execution times expose random behaviors. Further, one could combine SPARTA with other techniques, such as the ones aimed at reducing the signal-tonoise-ratio, for achieving even better protections. 


\section{Bibliography}

[AEP15] Amir Aminifar, Petru Eles, and Zebo Peng. Jfair: A scheduling algorithm to stabilize control applications. In IEEE Real-Time and Embedded Technology and Applications Symposium (RTAS), pages 63-72. IEEE, 2015.

[AW06] Krzysztof R. Apt and Mark Wallace. Constraint Logic Programming using ECLiPSe. Cambridge University Press, 2006.

[BAEP09] Min Bao, Alexandru Andrei, Petru Eles, and Zebo Peng. On-line thermal aware dynamic voltage scaling for energy optimization with frequency/temperature dependency consideration. In Design Automation Conference (DAC), pages 490-495, 2009.

[BB00] Thomas D Burd and Robert W Brodersen. Design issues for dynamic voltage scaling. In International Symposium on Low Power Electronics and Design (ISLPED), pages 9-14, 2000.

[BBMSS10] Sanjoy Baruah, Vincenzo Bonifaci, Alberto MarchettiSpaccamela, and Sebastian Stiller. Improved multiprocessor global schedulability analysis. Real-Time Systems, 46(1):3-24, 2010.

[BK98] Alex Biryukov and Eyal Kushilevitz. Improved cryptanalysis of RC5. In Advances in Cryptology (EUROCRYPT), pages 85-99. 1998. 
[BKL $\left.{ }^{+} 07\right]$ Andrey Bogdanov, Lars R Knudsen, Gregor Leander, Christof Paar, Axel Poschmann, Matthew JB Robshaw, Yannick Seurin, and Charlotte Vikkelsoe. PRESENT: An ultra-lightweight block cipher. Springer, 2007.

[BMR90] Sanjoy K Baruah, Aloysius K Mok, and Louis E Rosier. Preemptively scheduling hard-real-time sporadic tasks on one processor. In Real-Time Systems Symposium, pages 182-190. IEEE, 1990.

$\left[\mathrm{BMS}^{+}\right.$06] Lejla Batina, Nele Mentens, Kazuo Sakiyama, Bart Preneel, and Ingrid Verbauwhede. Low-cost elliptic curve cryptography for wireless sensor networks. In Security and Privacy in Ad-Hoc and Sensor Networks, pages 617. Springer, 2006.

[BS91] Eli Biham and Adi Shamir. Differential cryptanalysis of DES-like cryptosystems. Journal of CRYPTOLOGY, 4(1):3-72, 1991.

[But11] Giorgio C Buttazzo. Hard real-time computing systems: predictable scheduling algorithms and applications, volume 24. Springer, 2011.

[BVR $\left.{ }^{+} 13\right]$ Ali Galip Bayrak, Nikola Velickovic, Francesco Regazzoni, David Novo, Philip Brisk, and Paolo Ienne. An EDA-friendly protection scheme against side-channel attacks. In Design, Automation Test in Europe Conference Exhibition (DATE), pages 410-415, 2013.

[CAN91] CAN specification version 2.0. 1991.

[CAS08] Alvaro A Cárdenas, Saurabh Amin, and Shankar Sastry. Research challenges for the security of control systems. In HotSec, 2008.

[CAYM15] Paul Carsten, Todd R Andel, Mark Yampolskiy, and Jeffrey T McDonald. In-vehicle networks: Attacks, vulnerabilities, and proposed solutions. In 10th Annual Cyber 
and Information Security Research Conference, page 1. ACM, 2015.

[CB08] David Canright and Lejla Batina. A very compact "perfectly masked" S-box for AES. In Applied Cryptography and Network Security, pages 446-459. Springer, 2008.

[CEP04] Luis Alejandro Cortés, Petru Eles, and Zebo Peng. Quasi-static scheduling for real-time systems with hard and soft tasks. In Design, Automation and Test in Europe, page 21176, 2004.

[Cis14] Cisco. H.264 codec. https://github.com/cisco/ openh264, 2014. [Online]. Accessed: 2015-02-27.

[CK09] Jean-Sebastien Coron and Ilya Kizhvatov. An efficient method for random delay generation in embedded software. In Cryptographic Hardware and Embedded Systems (CHES), pages 156-170. Springer, 2009.

$\left[\mathrm{CMK}^{+} 11\right]$ Stephen Checkoway, Damon McCoy, Brian Kantor, Danny Anderson, Hovav Shacham, Stefan Savage, Karl Koscher, Alexei Czeskis, Franziska Roesner, and Tadayoshi Kohno. Comprehensive experimental analyses of automotive attack surfaces. In USENIX Security Symposium. San Francisco, 2011.

[CS07] Ioan Chisalita and Nahid Shahmehri. On the design of safety communication systems for vehicles. Systems, Man and Cybernetics, Part A: Systems and Humans, IEEE Transactions on, 37(6):933-945, 2007.

[DB92] Jutta Degener and Carsten Borman. GSM 06.10. http: //www.quut.com/gsm/gsm-1.0.13.tar.gz, 1992. [Online]. Accessed: 2015-02-21.

[DK08] Mohammad I Daoud and Nawwaf Kharma. A high performance algorithm for static task scheduling in heterogeneous distributed computing systems. Journal of Parallel and distributed computing, 68(4):399-409, 2008. 
[DLJ99] Bharat P Dave, Ganesh Lakshminarayana, and Niraj K Jha. COSYN: Hardware-software co-synthesis of heterogeneous distributed embedded systems. IEEE Transactions on Very Large Scale Integration (VLSI) Systems, 7(1):92-104, 1999.

[DPAM02] Kalyanmoy Deb, Amrit Pratap, Sameer Agarwal, and TAMT Meyarivan. A fast and elitist multiobjective genetic algorithm: NSGA-II. IEEE Transactions on Evolutionary Computation, 6:182-197, 2002.

[DPR00] Andreas Dandalis, Viktor K. Prasanna, and José D. P. Rolim. A comparative study of performance of AES final candidates using FPGAs. In Proc. Cryptographic Hardware and Embedded Systems Workshop, CHES, pages 125-140. Springer-Verlag, 2000.

[DR02] Joan Daemen and Vincent Rijmen. The design of Rijndael: AES-the Advanced Encryption Standard. Springer, 2002 .

[EPKD97] Petru Eles, Zebo Peng, Krzysztof Kuchcinski, and Alexa Doboli. System level hardware/software partitioning based on simulated annealing and tabu search. Design automation for embedded systems, 2(1):5-32, 1997.

[EPPD00] Petru Eles, Zebo Peng, Paul Pop, and Alex Doboli. Scheduling with bus access optimization for distributed embedded systems. IEEE Transaction Very Large Scale Integration System, 8(5):472-491, 2000.

[EYCP01] Adam J Elbirt, Wei Yip, Brendon Chetwynd, and Christof Paar. An FPGA-based performance evaluation of the AES block cipher candidate algorithm finalists. IEEE Transactions on Very Large Scale Integration (VLSI) Systems, 9(4):545 -557, aug. 2001.

[Fle05] Flexray communications system protocol specification version $2.1,2005$. 
[Geb06] Catherine H. Gebotys. A table masking countermeasure for low-energy secure embedded systems. IEEE transaction on VLSI Systems, 14:740-753, jul. 2006.

[GGS04] Philippe Golle, Dan Greene, and Jessica Staddon. Detecting and correcting malicious data in VANETs. In VANET '04: Proceedings of the 1st ACM international workshop on Vehicular ad hoc networks, pages 29-37, New York, NY, USA, 2004. ACM.

[Gre15] Andy Greenberg. Hackers remotely kill a Jeep on the highway - with me in it. http://www.wired.com/2015/ 07/hackers-remotely-kill-jeep-highway/, 2015. [Online]. Accessed: 2015-09-22.

[GYG $\left.{ }^{+} 08\right]$ Nan Guan, Wang Yi, Zonghua Gu, Qingxu Deng, and Ge Yu. New schedulability test conditions for nonpreemptive scheduling on multiprocessor platforms. In Real-Time Systems Symposium (RTSS), pages 137-146. IEEE, 2008.

[Hag97] Johan Hagman. Mp3 decoder. http://aminet.net/ mus/play/mpeg3play.lha, 1997. [Online]. Accessed: 2015-03-01.

[HCK06] Chia-Mei Hung, Jian-Jia Chen, and Tei-Wei Kuo. Energy-efficient real-time task scheduling for a DVS system with a Non-DVS processing element. In International Real-Time Systems Symposium (RTSS), pages 303-312, 2006.

[HFS98] Steven A. Hofmeyr, Stephanie Forrest, and Anil Somayaji. Intrusion detection using sequences of system calls. Journal of Computer Security, 6:151-180, 1998.

[HKD11] Tobias Hoppe, Stefan Kiltz, and Jana Dittmann. Security threats to automotive CAN networks-practical examples and selected short-term countermeasures. Relia- 
bility Engineering \&5 System Safety, 96(1):11 - 25, 2011. Special Issue on Safecomp 2008.

[HKHF11] Ondrej Hyncica, Pavel Kucera, Petr Honzik, and Petr Fiedler. Performance evaluation of symmetric cryptography in embedded systems. In International Conference on Intelligent Data Acquisition and Advanced Computing Systems (IDAACS), pages 277-282, 2011.

[HMGM13] Pengcheng Huang, Orlando Moreira, Kees Goossens, and Anca Molnos. Throughput-constrained voltage and frequency scaling for real-time heterogeneous multiprocessors. In The 28th Annual ACM Symposium on Applied Computing (SAC), pages 1517-1524, 2013.

[HS13] Michael Hutter and Peter Schwabe. $\mathrm{NaCl}$ on 8-bit AVR microcontrollers. In Progress in CryptologyAFRICACRYPT 2013, pages 156-172. Springer, 2013.

[HV08] Chen Huang and Frank Vahid. Dynamic coprocessor management for FPGA-enhanced compute platforms. In International Conference on Compilers, Architectures and Synthesis for Embedded Systems (CASES), pages 71-78, 2008.

[IJG98] Independent JPEG Group IJG. jpeg-6b. https: //quirkysoft.googlecode.com/files/jpegsrc.v6b. tar.gz, 1998. [Online]. Accessed: 2015-02-17.

[ILW06] Vinay M Igure, Sean A Laughter, and Ronald D Williams. Security issues in SCADA networks. Computers \& Security, 25(7):498-506, 2006.

[IPEP05] Viacheslav Izosimov, Paul Pop, Petru Eles, and Zebo Peng. Design optimization of time- and cost-constrained fault-tolerant distributed embedded systems. In Design, Automation and Test in Europe, pages 864-869, 2005.

[IPEP08] Viacheslav Izosimov, Paul Pop, Petru Eles, and Zebo Peng. Scheduling of fault-tolerant embedded systems 
with soft and hard timing constraints. In Proceedings of the conference on Design, automation and test in Europe, pages 915-920. ACM, 2008.

[JBEP14] Ke Jiang, Lejla Batina, Petru Eles, and Zebo Peng. Robustness analysis of real-time scheduling against differential power analysis attacks. In IEEE Computer Society Annual Symposium on VLSI (ISVLSI), pages 450-455, 2014 .

[JBEP15] Ke Jiang, Lejla Batina, Petru Eles, and Zebo Peng. The influence of real-time scheduling on differential power analysis attacks. In TRUDEVICE 2015: Workshop on Trustworthy Manufacturing and Utilization of Secure Devices (Co-located with DATE15), 2015.

[JEP11] Ke Jiang, Petru Eles, and Zebo Peng. Optimization of message encryption for distributed embedded systems with real-time constraints. In International Symposium on Design and Diagnostics of Electronic Circuits and Systems (DDECS), pages 243-248, 2011.

[JEP12] Ke Jiang, P. Eles, and Zebo Peng. Co-design techniques for distributed real-time embedded systems with communication security constraints. In Design, Automation Test in Europe (DATE), pages 947-952, 2012.

[JEP13] Ke Jiang, Petru Eles, and Zebo Peng. Optimization of secure embedded systems with dynamic task sets. In Design, Automation Test in Europe (DATE), 2013.

[JEP15] Ke Jiang, Petru Eles, and Zebo Peng. Power-aware design techniques of secure multi-mode embedded systems. ACM Transactions on Embedded Computing Systems (TECS), 2015.

[JEP $\left.{ }^{+} 16\right] \quad$ Ke Jiang, Petru Eles, Zebo Peng, Sudipta Chattopadhyay, and Lejla Batina. SPARTA: A scheduling policy for thwarting differential power analysis attacks. In Asia 
and South Pacific Design Automation Conference (ASPDAC), 2016.

[JJM12] Wei Jiang, Ke Jiang, and Yue Ma. Resource allocation of security-critical tasks with statistically guaranteed energy constraint. In International Conference on Embedded and Real-Time Computing Systems and Applications (RTCSA), pages 330-339, 2012.

[JJM14] Wei Jiang, Ke Jiang, and Yue Ma. Energy aware realtime scheduling policy with guaranteed security protection. In Asia and South Pacific Design Automation Conference (ASP-DAC), 2014.

[JJZM15] Wei Jiang, Ke Jiang, Xia Zhang, and Yue Ma. Energy optimization of security-critical real-time applications with guaranteed security protection. Journal of Systems Architecture (JSA), 61(7):282 - 292, 2015.

$\left[\mathrm{JLE}^{+} 13\right] \quad$ Ke Jiang, Adrian Lifa, Petru Eles, Zebo Peng, and Wei Jiang. Power-aware design of secure multi-mode realtime embedded systems with FPGA co-processors. In International Conference on Real-Time Networks and Systems (RTNS), 2013.

[KCR $\left.{ }^{+} 10\right]$ Karl Koscher, Alexei Czeskis, Franziska Roesner, Shwetak Patel, Tadayoshi Kohno, Stephen Checkoway, Damon McCoy, Brian Kantor, Danny Anderson, Hovav Shacham, and Stefan Savage. Experimental security analysis of a modern automobile. In 2010 IEEE Symposium on Security and Privacy (SP), pages 447-462. IEEE, 2010.

[KJJ99] Paul C. Kocher, Joshua Jaffe, and Benjamin Jun. Differential power analysis. In CRYPTO, pages 388-397, 1999. 
[KJV83] Scott Kirkpatrick, D. Gelatt Jr., and Mario P. Vecchi. Optimization by simulated annealing. Science, 220(4598):671-680, 1983.

$\left[\mathrm{KLH}^{+} 11\right]$ Markus Koester, Wayne Luk, Jens Hagemeyer, Mario Porrmann, and Ulrich Rückert. Design optimizations for tiled partially reconfigurable systems. IEEE Transactions on Very Large Scale Integration (VLSI) Systems, 19(6):1048-1061, 2011.

[KM01] Lars Knudsen and Willi Meier. Correlations in RC6 with a reduced number of rounds. In Fast Software Encryption, pages 94-108. 2001.

[LDKK08] Jiqiang Lu, Orr Dunkelman, Nathan Keller, and Jongsung Kim. New impossible differential attacks on AES. In Progress in Cryptology-INDOCRYPT, pages 279-293. 2008.

[Lee08] Edward A. Lee. Cyber physical systems: Design challenges. In IEEE International Symposium on Object Oriented Real-Time Distributed Computing (ISORC), pages 363-369. IEEE, 2008.

[LEP15] Adrian Lifa, Petru Eles, and Zebo Peng. On-the-fly energy minimization for multi-mode real-time systems on heterogeneous platforms. In IEEE/ACM Symposium on Embedded Systems for Real-Time Multimedia (ESTIMedia), October 2015.

[LH02] Gabriel Leen and Donal Heffernan. Expanding automotive electronic systems. Computer, 35(1):88-93, 2002.

[LJ97] Ulf Lindqvist and Erland Jonsson. How to systematically classify computer security intrusions. In IEEE Symposium on Security and Privacy, pages 154-163, 1997.

[LL73] Chung Laung Liu and James W Layland. Scheduling algorithms for multiprogramming in a hard-real-time en- 
vironment. Journal of the ACM (JACM), 20(1):46-61, 1973.

$\left[\mathrm{LPD}^{+} 14\right]$ Andrew Lukefahr, Shruti Padmanabha, Reetuparna Das, Ronald Dreslinski Jr, Thomas F Wenisch, and Scott Mahlke. Heterogeneous microarchitectures trump voltage scaling for low-power cores. In International Conference on Parallel Architectures and Compilation, pages 237-250, 2014.

[LSL ${ }^{+}$94] Jane WS Liu, Wei-Kuan Shih, Kwei-Jay Lin, Riccardo Bettati, and Jen-Yao Chung. Imprecise computations. Proceedings of the IEEE, 82(1):83-94, 1994.

[LSM99] Wenke Lee, Salvatore J Stolfo, and Kui W Mok. A data mining framework for building intrusion detection models. In IEEE Symposium on Security and Privacy, pages 120-132, 1999.

[LXY ${ }^{+}$09] Man Lin, Li Xu, L.T. Yang, Xiao Qin, Nenggan Zheng, Zhaohui Wu, and Meikang Qiu. Static security optimization for real-time systems. IEEE Transactions on Industrial Informatics (II), pages 22-37, 2009.

[Mar11] Peter Marwedel. Embedded system design: Embedded systems foundations of cyber-physical systems. Springer Science \& Business Media, 2011.

[MD11] Shohreh Sharif Mansouri and Elena Dubrova. A countermeasure against power analysis attacks for fsr-based stream ciphers. In Great Lakes Symposium on VLSI, pages 235-240. ACM, 2011.

[MD12] Shohreh Sharif Mansouri and Elena Dubrova. An architectural countermeasure against power analysis attacks for fsr-based stream ciphers. In Constructive Side-Channel Analysis and Secure Design, pages 54-68. Springer, 2012. 
[MFMB02] Steven M Martin, Krisztian Flautner, Trevor Mudge, and David Blaauw. Combined dynamic voltage scaling and adaptive body biasing for lower power microprocessors under dynamic workloads. In International Conference on Computer Aided Design (ICCAD), pages 721-725, 2002.

[ML09] Jingqing $\mathrm{Mu}$ and Roman Lysecky. Autonomous hardware/software partitioning and voltage/frequency scaling for low-power embedded systems. ACM Transactions on Design Automation of Electronic Systems (TODAES), 15(1):2:1-2:20, 2009.

[Moc14] Kosma Moczek. minmea, a lightweight GPS NMEA 0183 parser library. https://github.com/cloudyourcar/ minmea, 2014. [Online]. Accessed: 2015-02-27.

[MOP07] Stefan Mangard, Elisabeth Oswald, and Thomas Popp. Power analysis attacks: Revealing the secrets of smart cards. Springer, 2007.

[MSL $\left.{ }^{+} 15\right]$ Philipp Mundhenk, Sebastian Steinhorst, Martin Lukasiewycz, Suhaib A Fahmy, and Samarjit Chakraborty. Lightweight authentication for secure automotive networks. In Design, Automation and Test in Europe (DATE), pages 285-288, 2015.

[MZ05] Rabi N Mahapatra and Wei Zhao. An energy-efficient slack distribution technique for multimode distributed real-time embedded systems. IEEE Transactions on Parallel and Distributed Systems, 16(7):650-662, 2005.

$\left[\mathrm{NBB}^{+} 00\right]$ James Nechvatal, Elaine Barker, Lawrence Bassham, William Burr, and Morris Dworkin. Report on the development of the Advanced Encryption Standard (AES). Technical report, DTIC Document, 2000.

[OGOP04] Siddika Berna Ors, Frank Gurkaynak, Elisabeth Oswald, and Bart Preneel. Power-analysis attack on an ASIC 
AES implementation. In International Conference on Information Technology: Coding and Computing (ITCC), volume 2, pages 546-552, 2004.

[OH02] Hyunok Oh and Soonhoi Ha. Hardware-software cosynthesis of multi-mode multi-task embedded systems with real-time constraints. In International Symposium on Hardware/Software Codesign, pages 133-138. ACM, 2002.

$\left[\mathrm{PJJ}^{+} 16\right] \quad$ Xiong Pan, Wei Jiang, Ke Jiang, Liang Wen, and Qi Dong. Energy optimization of stochastic real-time applications with statistically guarantee of reliability constraints. In Asia and South Pacific Design Automation Conference (ASP-DAC), 2016.

[PJPM14] Anuj Pathania, Qing Jiao, Alok Prakash, and Tulika Mitra. Integrated CPU-GPU power management for 3D mobile games. In Design Automation Conference (DAC), 2014.

[Pop03] Paul Pop. Analysis and Synthesis of CommunicationIntensive Heterogeneous Real-Time Systems. PhD thesis, Linköping University, 2003.

[PP05] Bryan Parno and Adrian Perrig. Challenges in securing vehicular networks. In Workshop on Hot Topics in Networks (HotNets-IV), 2005.

[PP08] Krutartha Patel and Sri Parameswaran. SHIELD: a software hardware design methodology for security and reliability of MPSoCs. In Design Automation Conference (DAC), pages 858-861, 2008.

[PP10] Christof Paar and Jan Pelzl. Understanding Cryptography: A Textbook for Students and Practitioners. 2010.

[PPS $\left.{ }^{+} 13\right] \quad$ Sangyoung Park, Jaehyun Park, Donghwa Shin, Yanzhi Wang, Qing Xie, M. Pedram, and Naehyuck Chang. Ac- 
curate modeling of the delay and energy overhead of dynamic voltage and frequency scaling in modern microprocessors. IEEE Transactions on Computer-Aided Design of Integrated Circuits and Systems, 32(5):695-708, May 2013.

[PS01] Padmanabhan Pillai and Kang G Shin. Real-time dynamic voltage scaling for low-power embedded operating systems. In ACM SIGOPS Operating Systems Review, volume 35, pages 89-102, 2001.

[RH07] Maxim Raya and Jean-Pierre Hubaux. Securing vehicular ad hoc networks. Journal of Computer Security, 15(1):39-68, 2007.

[RLSS10] Ragunathan Raj Rajkumar, Insup Lee, Lui Sha, and John Stankovic. Cyber-physical systems: the next computing revolution. In Design Automation Conference (DAC), pages 731-736. ACM, 2010.

[RRC04] Srivaths Ravi, Anand Raghunathan, and Srimat Chakradhar. Tamper resistance mechanisms for secure embedded systems. International Conference on VLSI Design, pages 605-611, 2004.

[RRKH04] Srivaths Ravi, Anand Raghunathan, Paul Kocher, and Sunil Hattangady. Security in embedded systems: Design challenges. ACM Transactions on Embedded Computing Systems (TECS), 3:461-491, 2004.

[RRSY98] Ronald L Rivest, MJB Robshaw, Ray Sidney, and Yiqun Lisa Yin. The RC6 block cipher. In First Advanced Encryption Standard (AES) Conference, 1998.

[SAHE05] Marcus T. Schmitz, Bashir M. Al-Hashimi, and Petru Eles. Cosynthesis of energy-efficient multimode embedded systems with consideration of mode-execution probabilities. IEEE Transactions on Computer-Aided Design of Integrated Circuits and Systems, 24(2):153-169, 2005. 
[SAM04] S3C2440A 32-bit CMOS microcontroller user's manual revision 1. SAMSUNG Electronics Co. Ltd. 2004.

[SCG ${ }^{+}$03] G Edward Suh, Dwaine Clarke, Blaise Gassend, Marten Van Dijk, and Srinivas Devadas. AEGIS: architecture for tamper-evident and tamper-resistant processing. In International Conference on Supercomputing, pages 160171. ACM, 2003.

[SCT10] Andreas Schranzhofer, Jian-Jian Chen, and Lothar Thiele. Dynamic power-aware mapping of applications onto heterogeneous mpsoc platforms. IEEE Transactions on Industrial Informatics, 6(4):692-707, 2010.

[SEPC09] Soheil Samii, Petru Eles, Zebo Peng, and Anton Cervin. Quality-driven synthesis of embedded multi-mode control systems. In Design Automation Conference (DAC), pages 864-869, 2009.

[SM07] Karen Scarfone and Peter Mell. Guide to intrusion detection and prevention systems (IDPS). NIST special publication, 2007.

[SÖP04] François-Xavier Standaert, Sıddıka Berna Örs, and Bart Preneel. Power analysis of an FPGA. In Cryptographic Hardware and Embedded Systems (CHES), pages 30-44. Springer, 2004.

[SSL89] Brinkley Sprunt, Lui Sha, and John Lehoczky. Aperiodic task scheduling for hard-real-time systems. Real-Time Systems, 1(1):27-60, 1989.

[SV03] Patrick Schaumont and Ingrid Verbauwhede. Domainspecific codesign for embedded security. Computer, 36(4):68 - 74, april 2003.

[SXZ ${ }^{+}$06] Zili Shao, C. Xue, Q. Zhuge, M. Qiu, Bin Xiao, and E.H.M. Sha. Security protection and checking for embedded system integration against buffer overflow attacks via 
hardware/software. IEEE Transactions on Computers, pages 443-453, april 2006.

[TAV02] Kris Tiri, Moonmoon Akmal, and Ingrid Verbauwhede. A dynamic and differential CMOS logic with signal independent power consumption to withstand differential power analysis on smart cards. In European Solid-State Circuits Conference (ESSCIRC), pages 403-406, 2002.

[Ul175] Jeffrey D. Ullman. NP-complete scheduling problems. Journal of Computer and System sciences, 10(3):384393, 1975.

[Vau96] Serge Vaudenay. On the weak keys of Blowfish. In Fast Software Encryption, pages 27-32. 1996.

[Vee84] H.J.M. Veendrick. Short-circuit dissipation of static CMOS circuitry and its impact on the design of buffer circuits. IEEE Journal of Solid-State Circuits (SSC), 19(4):468-473, 1984.

[WAST12] Stefan Wildermann, Josef Angermeier, Eugen Sibirko, and Jürgen Teich. Placing multimode streaming applications on dynamically partially reconfigurable architectures. International Journal of Reconfigurable Computing, 2012:9, 2012.

[WDL14] Armin Wasicek, Patricia Derler, and Edward A. Lee. Aspect-oriented modeling of attacks in automotive cyber-physical systems. In Design Automation Conference, DAC, pages 1-6, 2014.

[WJJ+15] Liang Wen, Wei Jiang, Ke Jiang, Xia Zhang, Xiong Pan, and Keran Zhou. Detecting fault injection attacks on embedded real-time applications: A system-level perspective. In IEEE International Conference on High Performance Computing and Communications (HPCC), 2015.

[WMWZ12] Tongquan Wei, Piyush Mishra, Kaijie Wu, and Junlong Zhou. Quasi-static fault-tolerant scheduling schemes for 
energy-efficient hard real-time systems. Journal of Systems and Software, 85(6):1386-1399, 2012.

[WWP04] Marko Wolf, André Weimerskirch, and Christof Paar. Security in automotive bus systems. In Workshop on Embedded Security in Cars, 2004.

[WWP06] Marko Wolf, André Weimerskirch, and Christof Paar. Secure in-vehicle communication. In Embedded Security in Cars, pages 95-109. 2006.

[XP00] Jia Xu and David Lorge Parnas. Priority scheduling versus pre-run-time scheduling. Real-Time Systems, 18(1):7-23, 2000.

[XQ07] Tao Xie and Xiao Qin. Improving security for periodic tasks in embedded systems through scheduling. $A C M$ Transactions Embedded Computing Systems, 6, 2007.

[YD03] Dit-Yan Yeung and Yuxin Ding. Host-based intrusion detection using dynamic and static behavioral models. Pattern Recognition, 36:229-243, 2003.

$\left[\mathrm{YMC}^{+} 13\right]$ Man-Ki Yoon, S. Mohan, Jaesik Choi, Jung-Eun Kim, and Lui Sha. SecureCore: A multicore-based intrusion detection architecture for real-time embedded systems. In Real-Time and Embedded Technology and Applications Symposium (RTAS), pages 21-32, 2013.

[Zit01] Eckart Zitzler. Hypervolume metric calculation. ftp://ftp.tik.ee.ethz.ch/pub/people/zitzler/ hypervol.c, 2001. [Online]. Accessed: 2014-08-21.

[ZM05] Tom Zellar and Norman Mayersohn. Can a virus hitch a ride in your car? New York Times, March 13, 2005.

[ZSJ09] Jun Zhu, Ingo Sander, and Axel Jantsch. Buffer minimization of real-time streaming applications scheduling on hybrid CPU/FPGA architectures. In Design, Automation and Test in Europe, pages 1506-1511, 2009. 
[ZSJ10] Jun Zhu, Ingo Sander, and Axel Jantsch. Pareto efficient design for reconfigurable streaming applications on CPU/FPGAs. In Design, Automation and Test in Europe, pages 1035-1040, 2010.

[ZT98] Eckart Zitzler and Lothar Thiele. Multiobjective optimization using evolutionary algorithms - a comparative case study. In Conference on Parallel Problem Solving from Nature (PPSN V), pages 292-301, 1998.

[ZWF07] Wentao Zhang, Wenling Wu, and Dengguo Feng. New results on impossible differential cryptanalysis of reduced AES. In Information Security and Cryptology (ICISC), pages 239-250. 2007.

$\left[\right.$ ZZJ $\left.^{+}{ }^{13 a}\right]$ Xia Zhang, Jinyu Zhan, Wei Jiang, Yue Ma, and Ke Jiang. Design optimization of energy- and securitycritical distributed real-time embedded systems. In International Parallel and Distributed Processing Symposium Workshops \& PhD Forum (IPDPSW), 2013.

$\left[\right.$ ZZJ $^{+}{ }^{13 b]}$ Xia Zhang, Jinyu Zhan, Wei Jiang, Yue Ma, and Ke Jiang. Design optimization of security-sensitive mixed-criticality real-time embedded systems. In $1 s t$ Workshop on Real-Time Mixed Criticality Systems (ReTiMiCS), 2013. 


\section{Dissertations}

\section{Linköping Studies in Science and Technology Linköping Studies in Arts and Science Linköping Studies in Statistics Linköpings Studies in Information Science}

Linköping Studies in Science and Technology

No 14 Anders Haraldsson: A Program Manipulation System Based on Partial Evaluation, 1977, ISBN 917372-144-1.

No 17 Bengt Magnhagen: Probability Based Verification of Time Margins in Digital Designs, 1977, ISBN 91-7372157-3.

No 18 Mats Cedwall: Semantisk analys av processbeskrivningar i naturligt språk, 1977, ISBN 91-7372168-9.

No 22 Jaak Urmi: A Machine Independent LISP Compiler and its Implications for Ideal Hardware, 1978, ISBN 91-7372-188-3.

No 33 Tore Risch: Compilation of Multiple File Queries in a Meta-Database System 1978, ISBN 91-7372-232-4.

No 51 Erland Jungert: Synthesizing Database Structures from a User Oriented Data Model, 1980, ISBN 917372-387-8.

No 54 Sture Hägglund: Contributions to the Development of Methods and Tools for Interactive Design of Applications Software, 1980, ISBN 91-7372-404-1.

No 55 Pär Emanuelson: Performance Enhancement in a Well-Structured Pattern Matcher through Partial Evaluation, 1980, ISBN 91-7372-403-3.

No 58 Bengt Johnsson, Bertil Andersson: The HumanComputer Interface in Commercial Systems, 1981, ISBN 91-7372-414-9.

No 69 H. Jan Komorowski: A Specification of an Abstract Prolog Machine and its Application to Partial Evaluation, 1981, ISBN 91-7372-479-3.

No 71 René Reboh: Knowledge Engineering Techniques and Tools for Expert Systems, 1981, ISBN 91-7372489-0.

No 77 Östen Oskarsson: Mechanisms of Modifiability in large Softw are Systems, 1982, ISBN 91- 7372-527-7.

No 94 Hans Lunell: Code Generator Writing Systems, 1983, ISBN 91-7372-652-4.

No 97 Andrzej Lingas: Advances in Minimum Weight Triangulation, 1983, ISBN 91-7372-660-5.

No 109 Peter Fritzson: Tow ards a Distributed Programming Environment based on Incremental Compilation, 1984, ISBN 91-7372-801-2.

No 111 Erik Tengvald: The Design of Expert Planning Systems. An Experimental Operations Planning System for Turning, 1984, ISBN 91-7372- 805-5.

No 155 Christos Levcopoulos: Heuristics for Minimum Decompositions of Polygons, 1987, ISBN 91-7870133-3.

No 165 James W. Goodwin: A Theory and System for NonMonotonic Reasoning, 1987, ISBN 91-7870-183-X.

No 170 Zebo Peng: A Formal Methodology for Automated Synthesis of VLSI Systems, 1987, ISBN 91-7870-225-9.

No 174 Johan Fagerström: A Paradigm and System for Design of Distributed Systems, 1988, ISBN 91-7870301-8.

No 192 Dimiter Driankov: Tow ards a Many Valued Logic of Quantified Belief, 1988, ISBN 91-7870-374-3.
No 213 Lin Padgham: Non-Monotonic Inheritance for an Object Oriented Knowledge Base, 1989, ISBN 917870-485-5.

No 214 Tony Larsson: A Formal Hardware Description and Verification Method, 1989, ISBN 91-7870-517-7.

No 221 Michael Reinfrank: Fundamentals and Logical Foundations of Truth Maintenance, 1989, ISBN 917870-546-0.

No 239 Jonas Löwgren: Knowledge-Based Design Support and Discourse Management in User Interface Management Systems, 1991, ISBN 91-7870-720-X.

No 244 Henrik Eriksson: Meta-Tool Support for Know ledge Acquisition, 1991, ISBN 91-7870-746-3.

No 252 Peter Eklund: An Epistemic Approach to Interactive Design in Multiple Inheritance Hierarchies, 1991, ISBN 91-7870-784-6.

No 258 Patrick Doherty: NML3 - A Non-Monotonic Formalism with Explicit Defaults, 1991, ISBN 917870-816-8

No 260 Nahid Shahmehri: Generalized Algorithmic Debugging, 1991, ISBN 91-7870-828-1.

No 264 Nils Dahlbäck: Representation of DiscourseCognitive and Computational Aspects, 1992, ISBN 91-7870-850-8.

No 265 Ulf Nilsson: Abstract Interpretations and Abstract Machines: Contributions to a Methodology for the Implementation of Logic Programs, 1992, ISBN 917870-858-3

No 270 Ralph Rönnquist: Theory and Practice of Tensebound Object References, 1992, ISBN 91-7870-873-7.

No 273 Björn Fjellborg: Pipeline Extraction for VLSI Data Path Synthesis, 1992, ISBN 91-7870-880-X.

No 276 Staffan Bonnier: A Formal Basis for Horn Clause Logic with External Polymorphic Functions, 1992, ISBN 91-7870-896-6.

No 277 Kristian Sandahl: Developing Knowledge Management Systems with an Active Expert Methodology, 1992, ISBN 91-7870-897-4.

No 281 Christer Bäckström: Computational Complexity of Reasoning about Plans, 1992, ISBN 91-7870-979-2.

No 292 Mats Wirén: Studies in Incremental Natural Language Analysis, 1992, ISBN 91-7871-027-8.

No 297 Mariam Kamkar: Interprocedural Dynamic Slicing with Applications to Debugging and Testing, 1993, ISBN 91-7871-065-0.

No 302 Tingting Zhang: A Study in Diagnosis Using Classification and Defaults, 1993, ISBN 91-7871-078-2

No 312 Arne Jönsson: Dialogue Management for Natural Language Interfaces - An Empirical Approach, 1993, ISBN 91-7871-110-X.

No 338 Simin Nadjm-Tehrani: Reactive Systems in Physical Environments: Compositional Modelling and Framework for Verification, 1994, ISBN 91-7871-237-8.

No 371 Bengt Savén: Business Models for Decision Support and Learning. A Study of Discrete-Event Manufacturing Simulation at Asea/ ABB 1968-1993, 1995, ISBN 91-7871-494-X. 
No 375 Ulf Söderman: Conceptual Modelling of Mode Switching Physical Systems, 1995, ISBN 91-7871-5164.

No 383 Andreas Kågedal: Exploiting Groundness in Logic Programs, 1995, ISBN 91-7871-538-5.

No 396 George Fodor: Ontological Control, Description, Identification and Recovery from Problematic Control Situations, 1995, ISBN 91-7871-603-9.

No 413 Mikael Pettersson: Compiling Natural Semantics, 1995, ISBN 91-7871-641-1.

No 414 Xinli Gu: RT Level Testability Improvement by Testability Analysis and Transformations, 1996, ISBN 91-7871-654-3.

No 416 Hua Shu: Distributed Default Reasoning, 1996, ISBN 91-7871-665-9.

No 429 Jaime Villegas: Simulation Supported Industrial Training from an Organisational Learning Perspective - Development and Evaluation of the SSIT Method, 1996, ISBN 91-7871-700-0.

No 431 Peter Jonsson: Studies in Action Planning: Algorithms and Complexity, 1996, ISBN 91-7871-7043.

No 437 Johan Boye: Directional Types in Logic Programming, 1996, ISBN 91-7871-725-6.

No 439 Cecilia Sjöberg: Activities, Voices and Arenas: Participatory Design in Practice, 1996, ISBN 91-7871728-0.

No 448 Patrick Lambrix: Part-Whole Reasoning in Description Logics, 1996, ISBN 91-7871-820-1.

No 452 Kjell Orsborn: On Extensible and Object-Relational Database Technology for Finite Element Analysis Applications, 1996, ISBN 91-7871-827-9.

No 459 Olof Johansson: Development Environments for Complex Product Models, 1996, ISBN 91-7871-855-4.

No 461 Lena Strömbäck: User-Defined Constructions in Unification-Based Formalisms, 1997, ISBN 91-7871857-0.

No 462 Lars Degerstedt: Tabulation-based Logic Programming: A Multi-Level View of Query Answering, 1996, ISBN 91-7871-858-9.

No 475 Fredrik Nilsson: Strategi och ekonomisk styrning En studie av hur ekonomiska styrsystem utformas och används efter företagsförvärv, 1997, ISBN 91 7871-914-3.

No 480 Mikael Lindvall: An Empirical Study of Requirements-Driven Impact Analysis in Object-Oriented Softw are Evolution, 1997, ISBN 91-7871-927-5.

No 485 Göran Forslund: Opinion-Based Systems: The Cooperative Perspective on Knowledge-Based Decision Support, 1997, ISBN 91-7871-938-0.

No 494 Martin Sköld: Active Database Management Systems for Monitoring and Control, 1997, ISBN 91 7219-002-7.

No 495 Hans Olsén: Automatic Verification of Petri Nets in a CLP framew ork, 1997, ISBN 91-7219-011-6.

No 498 Thomas Drakengren: Algorithms and Complexity for Temporal and Spatial Formalisms, 1997, ISBN 917219-019-1.

No 502 Jakob Axelsson: Analysis and Synthesis of Heterogeneous Real-Time Systems, 1997, ISBN 91-7219-035-3.

No 503 Johan Ringström: Compiler Generation for DataParallel Programming Languages from Two-Level Semantics Specifications, 1997, ISBN 91-7219-045-0.

No 512 Anna Moberg: Närhet och distans - Studier av kommunikationsmönster i satellitkontor och flexibla kontor, 1997, ISBN 91-7219-119-8.
No 520 Mikael Ronström: Design and Modelling of a Parallel Data Server for Telecom Applications, 1998, ISBN 91-7219-169-4.

No 522 Niclas Ohlsson: Tow ards Effective Fault Prevention - An Empirical Study in Software Engineering, 1998, ISBN 91-7219-176-7.

No 526 Joachim Karlsson: A Systematic Approach for Prioritizing Software Requirements, 1998, ISBN 917219-184-8.

No 530 Henrik Nilsson: Declarative Debugging for Lazy Functional Languages, 1998, ISBN 91-7219-197-x.

No 555 Jonas Hallberg: Timing Issues in High-Level Synthesis, 1998, ISBN 91-7219-369-7.

No 561 Ling Lin: Management of 1-D Sequence Data - From Discrete to Continuous, 1999, ISBN 91-7219-402-2.

No 563 Eva L Ragnemalm: Student Modelling based on Collaborative Dialogue with a Learning Companion, 1999, ISBN 91-7219-412-X.

No 567 Jörgen Lindström: Does Distance matter? On geographical dispersion in organisations, 1999, ISBN 917219-439-1.

No 582 Vanja Josifovski: Design, Implementation and Evaluation of a Distributed Mediator System for Data Integration, 1999, ISBN 91-7219-482-0.

No 589 Rita Kovordányi: Modeling and Simulating Inhibitory Mechanisms in Mental Image Reinterpretation - Towards Cooperative HumanComputer Creativity, 1999, ISBN 91-7219-506-1.

No 592 Mikael Ericsson: Supporting the Use of Design Knowledge - An Assessment of Commenting Agents, 1999, ISBN 91-7219-532-0.

No 593 Lars Karlsson: Actions, Interactions and Narratives, 1999, ISBN 91-7219-534-7.

No 594 C. G. Mikael Johansson: Social and Organizational Aspects of Requirements Engineering Methods - A practice-oriented approach, 1999, ISBN 91-7219-541$\mathrm{X}$.

No 595 Jörgen Hansson: Value-Driven Multi-Class Overload Management in Real-Time Database Systems, 1999, ISBN 91-7219-542-8.

No 596 Niklas Hallberg: Incorporating User Values in the Design of Information Systems and Services in the Public Sector: A Methods Approach, 1999, ISBN 917219-543-6.

No 597 Vivian Vimarlund: An Economic Perspective on the Analysis of Impacts of Information Technology: From Case Studies in Health-Care tow ards General Models and Theories, 1999, ISBN 91-7219-544-4.

No 598 Johan Jenvald: Methods and Tools in ComputerSupported Taskforce Training, 1999, ISBN 91-7219547-9.

No 607 Magnus Merkel: Understanding and enhancing translation by parallel text processing, 1999, ISBN 91 7219-614-9.

No 611 Silvia Coradeschi: Anchoring symbols to sensory data, 1999, ISBN 91-7219-623-8.

No 613 Man Lin: Analysis and Synthesis of Reactive Systems: A Generic Layered Architecture Perspective, 1999, ISBN 91-7219-630-0.

No 618 Jimmy Tjäder: Systemimplementering i praktiken En studie av logiker i fyra projekt, 1999, ISBN 917219-657-2.

No 627 Vadim Engelson: Tools for Design, Interactive Simulation, and Visualization of Object-Oriented Models in Scientific Computing, 2000, ISBN 91-7219709-9. 
No 637 Esa Falkenroth: Database Technology for Control and Simulation, 2000, ISBN 91-7219-766-8.

No 639 Per-Arne Persson: Bringing Power and Knowledge Together: Information Systems Design for Autonomy and Control in Command Work, 2000, ISBN 91-7219796-X.

No 660 Erik Larsson: An Integrated System-Level Design for Testability Methodology, 2000, ISBN 91-7219-890-7.

No 688 Marcus Bjäreland: Model-based Execution Monitoring, 2001, ISBN 91-7373-016-5.

No 689 Joakim Gustafsson: Extending Temporal Action Logic, 2001, ISBN 91-7373-017-3.

No 720 Carl-Johan Petri: Organizational Information Provision - Managing Mandatory and Discretionary Use of Information Technology, 2001, ISBN-91-7373-1269.

No 724 Paul Scerri: Designing Agents for Systems with Adjustable Autonomy, 2001, ISBN 9173732079.

No 725 Tim Heyer: Semantic Inspection of Software Artifacts: From Theory to Practice, 2001, ISBN 91 73732087.

No 726 Pär Carlshamre: A Usability Perspective on Requirements Engineering - From Methodology to Product Development, 2001, ISBN 9173732125.

No 732 Juha Takkinen: From Information Management to Task Management in Electronic Mail, 2002, ISBN 91 73732583.

No 745 Johan Åberg: Live Help Systems: An Approach to Intelligent Help for Web Information Systems, 2002, ISBN 91-7373-311-3.

No 746 Rego Granlund: Monitoring Distributed Teamwork Training, 2002, ISBN 91-7373-312-1.

No 757 Henrik André-Jönsson: Indexing Strategies for Time Series Data, 2002, ISBN 917373-346-6.

No 747 Anneli Hagdahl: Development of IT-supported Interorganisational Collaboration - A Case Study in the Swedish Public Sector, 2002, ISBN 91-7373-314-8.

No 749 Sofie Pilemalm: Information Technology for NonProfit Organisations - Extended Participatory Design of an Information System for Trade Union Shop Stew ard s, 2002, ISBN 91-7373-318-0.

No 765 Stefan Holmlid: Adapting users: Towards a theory of use quality, 2002, ISBN 91-7373-397-0.

No 771 Magnus Morin: Multimedia Representations of Distributed Tactical Operations, 2002, ISBN 91-7373-4217.

No 772 Pawel Pietrzak: A Type-Based Framew ork for Locating Errors in Constraint Logic Programs, 2002, ISBN 91-7373-422-5.

No 758 Erik Berglund: Library Communication Among Programmers Worldwide, 2002, ISBN 91-7373-349-0.

No 774 Choong-ho Yi: Modelling Object-Oriented Dynamic Systems Using a Logic-Based Framew ork, 2002, ISBN 91-7373-424-1.

No 779 Mathias Broxvall: A Study in the Computational Complexity of Temporal Reasoning, 2002, ISBN 917373-440-3.

No 793 Asmus Pandikow: A Generic Principle for Enabling Interoperability of Structured and Object-Oriented Analysis and Design Tools, 2002, ISBN 91-7373-479-9.

No 785 Lars Hult: Publika Informationstjänster. En studie av den Internetbaserade encyklopedins bruksegenskaper, 2003, ISBN 91-7373-461-6.

No 800 Lars Taxén: A Framework for the Coordination of Complex Systems' Development, 2003, ISBN 917373-604-X
No 808 Klas Gäre: Tre perspektiv på förväntningar och förändringar i samband med införande av informationssystem, 2003, ISBN 91-7373-618-X.

No 821 Mikael Kindborg: Concurrent Comics programming of social agents by children, 2003, ISBN 91-7373-651-1.

No 823 Christina Ölvingson: On Development of Information Systems with GIS Functionality in Public Health Informatics: A Requirements Engineering Approach, 2003, ISBN 91-7373-656-2.

No 828 Tobias Ritzau: Memory Efficient Hard Real-Time Garbage Collection, 2003, ISBN 91-7373-666-X.

No 833 Paul Pop: Analysis and Synthesis of Communication-Intensive Heterogeneous Real-Time Systems, 2003, ISBN 91-7373-683-X.

No 852 Johan Moe: Observing the Dynamic Behaviour of Large Distributed Systems to Improve Development and Testing - An Empirical Study in Software Engineering, 2003, ISBN 91-7373-779-8.

No 867 Erik Herzog: An Approach to Systems Engineering Tool Data Representation and Exchange, 2004, ISBN 91-7373-929-4.

No 872 Aseel Berglund: Augmenting the Remote Control: Studies in Complex Information Navigation for Digital TV, 2004, ISBN 91-7373-940-5.

No 869 Jo Skåmedal: Telecommuting's Implications on Travel and Travel Patterns, 2004, ISBN 91-7373-935-9.

No 870 Linda Askenäs: The Roles of IT - Studies of Organising when Implementing and Using Enterprise Systems, 2004, ISBN 91-7373-936-7.

No 874 Annika Flycht-Eriksson: Design and Use of Ontologies in Information-Providing Dialogue Systems, 2004, ISBN 91-7373-947-2.

No 873 Peter Bunus: Debugging Techniques for EquationBased Languages, 2004, ISBN 91-7373-941-3.

No 876 Jonas Mellin: Resource-Predictable and Efficient Monitoring of Events, 2004, ISBN 91-7373-956-1.

No 883 Magnus Bång: Computing at the Speed of Paper: Ubiquitous Computing Environments for Healthcare Professionals, 2004, ISBN 91-7373-971-5

No 882 Robert Eklund: Disfluency in Swedish humanhuman and human-machine travel booking dialogues, 2004, ISBN 91-7373-966-9.

No 887 Anders Lindström: English and other Foreign Linguistic Elements in Spoken Swedish. Studies of Productive Processes and their Modelling using Finite-State Tools, 2004, ISBN 91-7373-981-2.

No 889 Zhiping Wang: Capacity-Constrained Production-inventory systems - Modelling and Analysis in both a traditional and an e-business context, 2004, ISBN 9185295-08-6.

No 893 Pernilla Qvarfordt: Eyes on Multimodal Interaction, 2004, ISBN 91-85295-30-2.

No 910 Magnus Kald: In the Borderland between Strategy and Management Control - Theoretical Framework and Empirical Evidence, 2004, ISBN 91-85295-82-5.

No 918 Jonas Lundberg: Shaping Electronic News: Genre Perspectives on Interaction Design, 2004, ISBN 9185297-14-3.

No 900 Mattias Arvola: Shades of use: The dynamics of interaction design for sociable use, 2004, ISBN 9185295-42-6.

No 920 Luis Alejandro Cortés: Verification and Scheduling Techniques for Real-Time Embedded Systems, 2004. ISBN 91-85297-21-6.

No 929 Diana Szentivanyi: Performance Studies of FaultTolerant Middleware, 2005, ISBN 91-85297-58-5. 
No 933 Mikael Cäker: Management Accounting as Constructing and Opposing Customer Focus: Three Case Studies on Management Accounting and Customer Relations, 2005, ISBN 91-85297-64-X.

No 937 Jonas Kvarnström: TALplanner and Other Extensions to Temporal Action Logic, 2005, ISBN 9185297-75-5.

No 938 Bourhane Kadmiry: Fuzzy Gain-Scheduled Visual Servoing for Unmanned Helicopter, 2005, ISBN 9185297-76-3.

No 945 Gert Jervan: Hybrid Built-In Self-Test and Test Generation Techniques for Digital Systems, 2005, ISBN: 91-85297-97-6.

No 946 Anders Arpteg: Intelligent Semi-Structured Information Extraction, 2005, ISBN 91-85297-98-4.

No 947 Ola Angelsmark: Constructing Algorithms for Constraint Satisfaction and Related Problems - Methods and Applications, 2005, ISBN 91-85297-99-2.

No 963 Calin Curescu: Utility-based Optimisation of Resource Allocation for Wireless Networks, 2005, ISBN 91-85457-07-8.

No 972 Björn Johansson: Joint Control in Dynamic Situations, 2005, ISBN 91-85457-31-0.

No 974 Dan Lawesson: An Approach to Diagnosability Analysis for Interacting Finite State Systems, 2005, ISBN 91-85457-39-6.

No 979 Claudiu Duma: Security and Trust Mechanisms for Groups in Distributed Services, 2005, ISBN 91-8545754-X.

No 983 Sorin Manolache: Analysis and Optimisation of Real-Time Systems with Stochastic Behaviour, 2005, ISBN 91-85457-60-4.

No 986 Yuxiao Zhao: Standards-Based Application Integration for Business-to-Business Communications, 2005, ISBN 91-85457-66-3.

No 1004 Patrik Haslum: Admissible Heuristics for Automated Planning, 2006, ISBN 91-85497-28-2.

No 1005 Aleksandra Tešanovic: Developing Reusable and Reconfigurable Real-Time Software using Aspects and Components, 2006, ISBN 91-85497-29-0.

No 1008 David Dinka: Role, Identity and Work: Extending the design and development agenda, 2006, ISBN 9185497-42-8.

No 1009 Iakov Nakhimovski: Contributions to the Modeling and Simulation of Mechanical Systems with Detailed Contact Analysis, 2006, ISBN 91-85497-43-X.

No 1013 Wilhelm Dahllöf: Exact Algorithms for Exact Satisfiability Problems, 2006, ISBN 91-85523-97-6.

No 1016 Levon Saldamli: PDEModelica - A High-Level Language for Modeling with Partial Differential Equations, 2006, ISBN 91-85523-84-4.

No 1017 Daniel Karlsson: Verification of Component-based Embedded System Designs, 2006, ISBN 91-85523-79-8

No 1018 Ioan Chisalita: Communication and Networking Techniques for Traffic Safety Systems, 2006, ISBN 9185523-77-1.

No 1019 Tarja Susi: The Puzzle of Social Activity - The Significance of Tools in Cognition and Cooperation, 2006, ISBN 91-85523-71-2.

No 1021 Andrzej Bednarski: Integrated Optimal Code Generation for Digital Signal Processors, 2006, ISBN 9185523-69-0.

No 1022 Peter Aronsson: Automatic Parallelization of Equation-Based Simulation Programs, 2006, ISBN 9185523-68-2.
No 1030 Robert Nilsson: A Mutation-based Framework for Automated Testing of Timeliness, 2006, ISBN 9185523-35-6.

No 1034 Jon Edvardsson: Techniques for Automatic Generation of Tests from Programs and Specifications, 2006, ISBN 91-85523-31-3.

No 1035 Vaida Jakoniene: Integration of Biological Data, 2006, ISBN 91-85523-28-3.

No 1045 Genevieve Gorrell: Generalized Hebbian Algorithms for Dimensionality Reduction in Natural Language Processing, 2006, ISBN 91-85643-88-2.

No 1051 Yu-Hsing Huang: Having a New Pair of Glasses Applying Systemic Accident Models on Road Safety, 2006, ISBN 91-85643-64-5.

No 1054 Åsa Hedenskog: Perceive those things which cannot be seen - A Cognitive Systems Engineering perspective on requirements management, 2006, ISBN 91-85643-57-2.

No 1061 Cécile Åberg: An Evaluation Platform for Semantic Web Technology, 2007, ISBN 91-85643-31-9.

No 1073 Mats Grindal: Handling Combinatorial Explosion in Software Testing, 2007, ISBN 978-91-85715-74-9.

No 1075 Almut Herzog: Usable Security Policies for Runtime Environments, 2007, ISBN 978-91-85715-65-7.

No 1079 Magnus Wahlström: Algorithms, measures, and upper bounds for Satisfiability and related problems, 2007, ISBN 978-91-85715-55-8.

No 1083 Jesper Andersson: Dynamic Software Architectures, 2007, ISBN 978-91-85715-46-6.

No 1086 Ulf Johansson: Obtaining Accurate and Comprehensible Data Mining Models - An Evolutionary Approach, 2007, ISBN 978-91-85715-34-3.

No 1089 Traian Pop: Analysis and Optimisation of Distributed Embedded Systems with Heterogeneous Scheduling Policies, 2007, ISBN 978-91-85715-27-5.

No 1091 Gustav Nordh: Complexity Dichotomies for CSPrelated Problems, 2007, ISBN 978-91-85715-20-6.

No 1106 Per Ola Kristensson: Discrete and Continuous Shape Writing for Text Entry and Control, 2007, ISBN 97891-85831-77-7.

No 1110 He Tan: Aligning Biomedical Ontologies, 2007, ISBN 978-91-85831-56-2.

No 1112 Jessica Lindblom: Minding the body - Interacting socially through embodied action, 2007, ISBN 978-9185831-48-7.

No 1113 Pontus Wärnestål: Dialogue Behavior Management in Conversational Recommender Systems, 2007, ISBN 978-91-85831-47-0.

No 1120 Thomas Gustafsson: Management of Real-Time Data Consistency and Transient Overloads in Embedded Systems, 2007, ISBN 978-91-85831-33-3.

No 1127 Alexandru Andrei: Energy Efficient and Predictable Design of Real-time Embedded Systems, 2007, ISBN 978-91-85831-06-7.

No 1139 Per Wikberg: Eliciting Knowledge from Experts in Modeling of Complex Systems: Managing Variation and Interactions, 2007, ISBN 978-91-85895-66-3.

No 1143 Mehdi Amirijoo: QoS Control of Real-Time Data Services under Uncertain Workload, 2007, ISBN 978 91-85895-49-6.

No 1150 Sanny Syberfeldt: Optimistic Replication with Forward Conflict Resolution in Distributed Real-Time Databases, 2007, ISBN 978-91-85895-27-4.

No 1155 Beatrice Alenljung: Envisioning a Future Decision Support System for Requirements Engineering - A Holistic and Human-centred Perspective, 2008, ISBN 978-91-85895-11-3. 
No 1156 Artur Wilk: Types for XML with Application to Xcerpt, 2008, ISBN 978-91-85895-08-3.

No 1183 Adrian Pop: Integrated Model-Driven Development Environments for Equation-Based Object-Oriented Languages, 2008, ISBN 978-91-7393-895-2.

No 1185 Jörgen Skågeby: Gifting Technologies Ethnographic Studies of End-users and Social Media Sharing, 2008, ISBN 978-91-7393-892-1.

No 1187 Imad-Eldin Ali Abugessaisa: Analytical tools and information-sharing methods supporting road safety organizations, 2008, ISBN 978-91-7393-887-7.

No 1204 H. Joe Steinhauer: A Representation Scheme for Description and Reconstruction of Object Configurations Based on Qualitative Relations, 2008, ISBN 978-91-7393-823-5.

No 1222 Anders Larsson: Test Optimization for Core-based System-on-Chip, 2008, ISBN 978-91-7393-768-9.

No 1238 Andreas Borg: Processes and Models for Capacity Requirements in Telecommunication Systems, 2009, ISBN 978-91-7393-700-9.

No 1240 Fredrik Heintz: DyKnow: A Stream-Based Knowledge Processing Middleware Framework, 2009, ISBN 978-91-7393-696-5.

No 1241 Birgitta Lindström: Testability of Dynamic RealTime System s, 2009, ISBN 978-91-7393-695-8.

No 1244 Eva Blomqvist: Semi-automatic Ontology Construction based on Patterns, 2009, ISBN 978-91-7393-683-5.

No 1249 Rogier Woltjer: Functional Modeling of Constraint Management in Aviation Safety and Command and Control, 2009, ISBN 978-91-7393-659-0.

No 1260 Gianpaolo Conte: Vision-Based Localization and Guid ance for Unmanned Aerial Vehicles, 2009, ISBN 978-91-7393-603-3.

No 1262 AnnMarie Ericsson: Enabling Tool Support for Formal Analysis of ECA Rules, 2009, ISBN 978-91-7393598-2.

No 1266 Jiri Trnka: Exploring Tactical Command and Control: A Role-Playing Simulation Approach, 2009, ISBN 978-91-7393-571-5.

No 1268 Bahlol Rahimi: Supporting Collaborative Work through ICT - How End-users Think of and Adopt Integrated Health Information Systems, 2009, ISBN 978-91-7393-550-0.

No 1274 Fredrik Kuivinen: Algorithms and Hardness Results for Some Valued CSPs, 2009, ISBN 978-91-7393-525-8.

No 1281 Gunnar Mathiason: Virtual Full Replication for Scalable Distributed Real-Time Databases, 2009, ISBN 978-91-7393-503-6.

No 1290 Viacheslav Izosimov: Scheduling and Optimization of Fault-Tolerant Distributed Embedded Systems, 2009, ISBN 978-91-7393-482-4.

No 1294 Johan Thapper: Aspects of a Constraint Optimisation Problem, 2010, ISBN 978-91-7393-464-0.

No 1306 Susanna Nilsson: Augmentation in the Wild: User Centered Development and Evaluation of Augmented Reality Applications, 2010, ISBN 978-917393-416-9.

No 1313 Christer Thörn: On the Quality of Feature Models, 2010, ISBN 978-91-7393-394-0.

No 1321 Zhiyuan He: Temperature Aware and DefectProbability Driven Test Scheduling for System-onChip, 2010, ISBN 978-91-7393-378-0.

No 1333 David Broman: Meta-Languages and Semantics for Equation-Based Modeling and Simulation, 2010, ISBN 978-91-7393-335-3.

No 1337 Alexander Siemers: Contributions to Modelling and Visualisation of Multibody Systems Simulations with
Detailed Contact Analysis, 2010, ISBN 978-91-7393317-9.

No 1354 Mikael Asplund: Disconnected Discoveries: Availability Studies in Partitioned Networks, 2010, ISBN 978-91-7393-278-3.

No 1359 Jana Rambusch: Mind Games Extended: Understanding Gameplay as Situated Activity, 2010, ISBN 978-91-7393-252-3.

No 1373 Sonia Sangari: Head Movement Correlates to Focus Assignment in Swedish,2011,ISBN 978-91-7393-154-0.

No 1374 Jan-Erik Källhammer: Using False Alarms when Developing Automotive Active Safety Systems, 2011, ISBN 978-91-7393-153-3.

No 1375 Mattias Eriksson: Integrated Code Generation, 2011, ISBN 978-91-7393-147-2.

No 1381 Ola Leifler: Affordances and Constraints of Intelligent Decision Support for Military Command and Control - Three Case Studies of Support Systems, 2011, ISBN 978-91-7393-133-5.

No 1386 Soheil Samii: Quality-Driven Synthesis and Optimization of Embedded Control Systems, 2011, ISBN 978-91-7393-102-1.

No 1419 Erik Kuiper: Geographic Routing in Intermittentlyconnected Mobile Ad Hoc Networks: Algorithms and Performance Models, 2012, ISBN 978-91-7519981-8.

No 1451 Sara Stymne: Text Harmonization Strategies for Phrase-Based Statistical Machine Translation, 2012, ISBN 978-91-7519-887-3.

No 1455 Alberto Montebelli: Modeling the Role of Energy Management in Embodied Cognition, 2012, ISBN 978-91-7519-882-8.

No 1465 Mohammad Saifullah: Biologically-Based Interactive Neural Network Models for Visual Attention and Object Recognition, 2012, ISBN 978-91-7519-838-5.

No 1490 Tomas Bengtsson: Testing and Logic Optimization Techniques for Systems on Chip, 2012, ISBN 978-917519-742-5.

No 1481 David Byers: Improving Software Security by Preventing Known Vulnerabilities, 2012, ISBN 97891-7519-784-5.

No 1496 Tommy Färnqvist: Exploiting Structure in CSPrelated Problems, 2013, ISBN 978-91-7519-711-1.

No 1503 John Wilander: Contributions to Specification, Implementation, and Execution of Secure Software, 2013, ISBN 978-91-7519-681-7.

No 1506 Magnus Ingmarsson: Creating and Enabling the Useful Service Discovery Experience, 2013, ISBN 97891-7519-662-6.

No 1547 Wladimir Schamai: Model-Based Verification of Dynamic System Behavior against Requirements: Method, Language, and Tool, 2013, ISBN 978-917519-505-6.

No 1551 Henrik Svensson: Simulations, 2013, ISBN 978-917519-491-2.

No 1559 Sergiu Rafiliu: Stability of Adaptive Distributed Real-Time Systems with Dynamic Resource Management, 2013, ISBN 978-91-7519-471-4.

No 1581 Usman Dastgeer: Performance-aware Component Composition for GPU-based Systems, 2014, ISBN 978-91-7519-383-0.

No 1602 Cai Li: Reinforcement Learning of Locomotion based on Central Pattern Generators, 2014, ISBN 978-917519-313-7.

No 1652 Roland Samlaus: An Integrated Development Environment with Enhanced Domain-Specific 
Interactive Model Validation, 2015, ISBN 978-917519-090-7.

No 1663 Hannes Uppman: On Some Combinatorial Optimization Problems: Algorithms and Complexity, 2015, ISBN 978-91-7519-072-3.

No 1664 Martin Sjölund: Tools and Methods for Analysis, Debugging, and Performance Improvement of Equation-Based Models, 2015, ISBN 978-91-7519-071-6.

No 1666 Kristian Stavåker: Contributions to Simulation of Modelica Models on Data-Parallel Multi-Core Architectures, 2015, ISBN 978-91-7519-068-6.

No 1680 Adrian Lifa: Hardware/ Software Codesign of Embedded Systems with Reconfigurable and Heterogeneous Platforms, 2015, ISBN 978-91-7519-0402.

No 1685 Bogdan Tanasa: Timing Analysis of Distributed Embedded Systems with Stochastic Workload and Reliability Constraints, 2015, ISBN 978-91-7519-022-8.

No 1691 Håkan Warnquist: Troubleshooting Trucks Automated Planning and Diagnosis, 2015, ISBN 97891-7685-993-3.

No 1702 Nima Aghaee: Thermal Issues in Testing of Advanced Systems on Chip, 2015, ISBN 978-91-7685949-0.

No 1715 Maria Vasilevskaya: Security in Embedded Systems: A Model-Based Approach with Risk Metrics, 2015, ISBN 978-91-7685-917-9.

No 1729 Ke Jiang: Security-Driven Design of Real-Time Embedded System, 2016, ISBN 978-91-7685-884-4.

No 1733 Victor Lagerkvist: Strong Partial Clones and the Complexity of Constraint Satisfaction Problems: Limitations and Applications, 2016, ISBN 978-91-7685856-1.

No 1734 Chandan Roy: An Informed System Development Approach to Tropical Cyclone Track and Intensity Forecasting, 2016, ISBN 978-91-7685-854-7.

Linköping Studies in Arts and Science

No 504 Ing-Marie Jonsson: Social and Emotional Characteristics of Speech-based In-Vehicle Information Systems: Impact on Attitude and Driving Behaviour, 2009, ISBN 978-91-7393-478-7.

No 586 Fabian Segelström: Stakeholder Engagement for Service Design: How service designers identify and communicate insights, 2013, ISBN 978-91-7519-554-4.

No 618 Johan Blomkvist: Representing Future Situations of Service: Prototyping in Service Design, 2014, ISBN 978-91-7519-343-4.

No 620 Marcus Mast: Human-Robot Interaction for SemiAutonomous Assistive Robots, 2014, ISBN 978-917519-319-9.

\section{Linköping Studies in Statistics}

No 9 Davood Shahsavani: Computer Experiments Designed to Explore and Approximate Complex Deterministic Models, 2008, ISBN 978-91-7393-976-8.

No 10 Karl Wahlin: Roadmap for Trend Detection and Assessment of Data Quality, 2008, ISBN 978-91-7393792-4.

No 11 Oleg Sysoev: Monotonic regression for large multivariate datasets, 2010, ISBN 978-91-7393-412-1.

No 13 Agné Burauskaite-Harju: Characterizing Temporal Change and Inter-Site Correlations in Daily and Subdaily Precipitation Extremes, 2011, ISBN 978-91-7393110-6.

\section{Linköping Studies in Information Science}

No 1 Karin Axelsson: Metodisk systemstrukturering- att skapa samstämmighet mellan informationssystemarkitektur och verksamhet, 1998. ISBN-9172-19-296-8.

No 2 Stefan Cronholm: Metodverktyg och användbarhet en studie av datorstödd metodbaserad systemutveckling, 1998, ISBN-9172-19-299-2.

No 3 Anders Avdic: Användare och utvecklare - om anveckling med kalkylprogram, 1999. ISBN-91-7219606-8.

No 4 Owen Eriksson: Kommunikationskvalitet hos informationssystem och affärsprocesser, 2000, ISBN 917219-811-7.

No 5 Mikael Lind: Från system till process - kriterier för processbestämning vid verksamhetsanalys, 2001, ISBN 91-7373-067-X.

No 6 Ulf Melin: Koordination och informationssystem i företag och nätverk, 2002, ISBN 91-7373-278-8.

No 7 Pär J. Ågerfalk: Information Systems Actability - Understanding Information Technology as a Tool for Business Action and Communication, 2003, ISBN 917373-628-7.

No 8 Ulf Seigerroth: Att förstå och förändra systemutvecklingsverksamheter - en taxonomi för metautveckling, 2003, ISBN91-7373-736-4.

No 9 Karin Hedström: Spår av datoriseringens värden Effekter av IT i äldreomsorg, 2004, ISBN 91-7373-9634.

No 10 Ewa Braf: Knowledge Demanded for Action Studies on Knowledge Mediation in Organisations, 2004, ISBN 91-85295-47-7.

No 11 Fredrik Karlsson: Method Configuration method and computerized tool support, 2005, ISBN 91-8529748-8.

No 12 Malin Nordström: Styrbar systemförvaltning - Att organisera systemförvaltningsverksamhet med hjälp av effektiva förvaltningsobjekt, 2005, ISBN 91-8529760-7.

No 13 Stefan Holgersson: Yrke: POLIS - Yrkeskunskap, motivation, IT-system och andra förutsättningar för polisarbete, 2005, ISBN 91-85299-43-X.

No 14 Benneth Christiansson, Marie-Therese Christiansson: Mötet mellan process och komponent - mot ett ramverk för en verksamhetsnära kravspecifikation vid anskaffning av komponentbaserade informationssystem, 2006, ISBN 91-85643$22-\mathrm{X}$. 